\title{
HOMOGENEOUS INTEGRABLE LEGENDRIAN CONTACT STRUCTURES IN DIMENSION FIVE
}

\author{
BORIS DOUBROV, ALEXANDR MEDVEDEV, AND DENNIS THE
}

\begin{abstract}
We consider Legendrian contact structures on odd-dimensional complex analytic manifolds. We are particularly interested in integrable structures, which can be encoded by compatible complete systems of second order PDEs on a scalar function of many independent variables and considered up to point transformations. Using the techniques of parabolic differential geometry, we compute the associated regular, normal Cartan connection and give explicit formulas for the harmonic part of the curvature. The PDE system is trivializable by means of point transformations if and only if the harmonic curvature vanishes identically.

In dimension five, the harmonic curvature takes the form of a binary quartic field, so there is a Petrov classification based on its root type. We give a complete local classification of all fivedimensional integrable Legendrian contact structures whose symmetry algebra is transitive on the manifold and has at least one-dimensional isotropy algebra at any point.
\end{abstract}

\section{INTRODUCTION}

A Legendrian contact structure $(M ; E, F)$ is defined to be a splitting of a contact distribution $C$ (on an odd-dimensional manifold $M$ ) into the direct sum of two subdistributions $E, F$ that are maximally isotropic with respect to the naturally defined conformal symplectic structure on $C$. Such structures can be treated in both the real smooth and complex analytic categories. In the current paper, we assume that all our manifolds and related objects are complex analytic, although many results are also valid in the smooth category.

We shall exclusively deal with integrable Legendrian contact structures (or just ILC structures), which means that both isotropic subdistributions are completely integrable. The main sources of ILC structures are compatible complete systems of 2nd order PDEs on one unknown function of several variables (considered up to point transformations), i.e.

$$
\frac{\partial^{2} u}{\partial x^{i} \partial x^{j}}=f_{i j}(x, u, \partial u), \quad 1 \leq i, j \leq n
$$

and the complexifications of (Levi-nondegenerate) CR structures of codimension 1.

The smallest dimension of a manifold with a Legendrian contact structure is 3 . In this dimension both isotropic subdistributions are 1-dimensional and are automatically completely integrable. The corresponding ILC structures can be encoded by a single 2nd order ODE and have been well-studied starting from the pioneering work of Tresse [17] (see also [2, 6, 15]). Their real counterpart, CR structures on 3-dimensional real hypersurfaces in $\mathbb{C}^{2}$, have also been well-studied starting from the classical works of Élie Cartan [7, 8].

Legendrian contact structures belong to the class of so-called parabolic geometries. In particular, they enjoy a number of important properties derived from the general theory of parabolic geometries [4]: the existence of a natural Cartan connection, description of the principal invariants in terms of the representation theory of simple Lie algebras, finite-dimensional symmetry algebras, and the classification of submaximal symmetry dimensions [12]. Legendrian contact structures

2010 Mathematics Subject Classification. Primary: 58J70; Secondary: 35A30, 53A40, 53B15, 53D10, 22E46.

Key words and phrases. Legendrian structures, symmetry algebra, curvature module, multiply transitive, complete systems of PDEs. 
are modeled by the flag variety $\operatorname{Flag}_{1, n+1}\left(\mathbb{C}^{n+2}\right)$ of pairs of incident lines and hyperplanes in $\mathbb{C}^{n+1}$ equipped with a natural action of $\operatorname{PGL}(n+2, \mathbb{C})$.

We note that in [16], Takeuchi studied the special class of Legendrian contact structures that are induced on the projective cotangent bundle $M=\mathcal{P}\left(T^{*} N\right)$ from a projective structure $(N,[\nabla])$. With the sole exception of the flat model, this induced structure on $M$ is never an ILC structure. Thus, his study is transverse to our study here.

In the current paper we are mainly interested in the classification of multiply transitive ILC structures in dimension 5. The term "multiply transitive" means that the symmetry algebra of the ILC structure should be transitive on the manifold and should have a non-trivial isotropy subalgebra (i.e. at least one-dimensional) at each point. As our study here is local in nature, we may as well require these conditions in an open subset of the manifold.

In dimension 3, all multiply transitive ILC structures are flat. This reflects a well-known fact that any 2 nd order ODE is either equivalent to the trivial equation $u^{\prime \prime}(x)=0$ and has 8-dimensional symmetry algebra, or its symmetry algebra is at most 3-dimensional. In dimension 5 this is no longer the case, as, for example, the submaximally symmetric ILC structures have symmetry algebras of dimension 8 and are multiply transitive [12]. In fact, all ILC structures with 8 symmetries are locally equivalent. This leaves us with the classification of ILC structures with 6- and 7dimensional symmetry. A similar classification of integrable CR-manifolds in dimension 5 with transitive symmetry algebras of dimension 7 was done by A.V. Loboda [13, 14].

As in the case of the geometry of scalar 2nd order ODEs, complete systems of 2nd order PDEs also admit a notion of duality that swaps the set of dependent and independent variables with the space of constants of integration parametrizing the generic solution. This corresponds to swapping the two isotropic distributions defining the ILC structure. We classify ILC structures up to this duality and indicate which structures are self-dual, i.e. locally contact equivalent to their dual.

In his famous 1910 paper [5], Élie Cartan studied the geometry of rank two distributions on 5 -manifolds having generic growth vector $(2,3,5)$. For such structures, Cartan solved the local equivalence problem and obtained a classification of all multiply transitive models 1$]$ While the equivalence problem was solved by means of Cartan's equivalence method [10], we bypass this step in our study of ILC structures by using the full power of parabolic geometry. Indeed, representation theory is used to quickly construct the full curvature module and set up the structure equations for the (regular, normal) Cartan geometry. Our classification of multiply transitive ILC structures implements Cartan's technique, which we refer to as Cartan's reduction method.

There is another striking similarity between ILC structures in dimension 5 and $(2,3,5)$ distributions. In both cases the fundamental invariant is represented by a single binary quartic. Similar to the Petrov classification for the Weyl curvature tensor in Lorentzian (conformal) geometry, we classify ILC structures in dimension 5 by the number and multiplicity of roots of this quartic. We also prove that non-flat multiply transitive structures may only have type $\mathrm{N}$ (a single root of multiplicity 4 ), type D (two roots of multiplicity 2), or type III (one simple root and one root of multiplicity 3). This is quite similar to Cartan's result [5] that all multiply transitive $(2,3,5)$-distributions have either type $\mathrm{N}$ or type $\mathrm{D}$. We identify the maximal symmetry dimension for each Petrov type in Theorem 3.1 .

The main result of our paper can be summarized as follows:

Theorem 1.1. Any multiply transitive ILC structure in dimension 5 is locally equivalent to the ILC structure defined by one of PDE models in Table 1.1 or its dual.

Remark 1.2. We denote by $u_{11}, u_{12}, u_{22}$ the second order partial derivatives of the unknown function $u$, and use the notation $p=u_{1}, q=u_{2}$ for the first order derivatives.

\footnotetext{
${ }^{1}$ One inadvertent omission from Cartan's list was recently discovered in 9.
} 
TABLE 1.1. Classification of all multiply transitive ILC structures in dimension 5

\begin{tabular}{|l|c|c|c|c|l|}
\hline Model & $\mathrm{SD}$ & $u_{11}$ & $u_{12}$ & $u_{22}$ & Remarks \\
\hline \hline O.15 & $\checkmark$ & 0 & 0 & 0 & flat model \\
\hline N.8 & $\checkmark$ & $q^{2}$ & 0 & 0 & unique submaximal \\
N.7-1 & $\times$ & $q^{2} \mathcal{G}_{\kappa}(x)$ & 0 & 0 & $\kappa \in \mathbb{C}_{\infty} \backslash\{0,-3\} ; \kappa \sim-3-\kappa$ \\
N.7-2 & $\checkmark$ & $\frac{1}{q}$ & 1 & 0 & \\
N.6-1 & $\checkmark$ & $\mathcal{F}_{\mu}(q)$ & 1 & 0 & $\mu \in \mathbb{C} \backslash\{-1,2\}$ \\
N.6-2 & $*$ & $\mathcal{F}_{\mu}(q) \mathcal{G}_{\kappa}(x)$ & 0 & 0 & $\mu \in \mathbb{C}_{\infty} \backslash\{-1,2\}, \kappa \in \mathbb{C}_{\infty} \backslash\{0,-3\} ;$ \\
& & & 0 & $\lambda q^{2}$ & $\lambda \in \mathbb{C} \backslash\{-1\} ; \lambda \sim \frac{1}{\lambda}$ for $\lambda \neq 0$ \\
\hline D.7 & $\checkmark$ & $p^{2}$ & $p-\frac{q^{2}}{2}$ & $\mu \in \mathbb{C} \mathbb{C}_{\infty} \backslash\{0,1,2\}$ \\
D.6-1 & $\checkmark$ & $p^{2}-\frac{q^{4}}{4}$ & $q\left(p-\frac{q^{2}}{2}\right)$ & 0 & $R=\frac{\sqrt{u-p q}}{u^{3 / 2}}, \lambda \in \mathbb{C} \backslash\left\{0, \pm \frac{1}{2}\right\} ; \lambda \sim-\lambda$ \\
D.6-2 & $\checkmark$ & $\mathcal{G}_{\mu}(p)$ & 0 & $\lambda q^{2} R$ & \\
D.6-3 & $\checkmark$ & $\lambda p^{2} R$ & $1+\lambda(p q-2 u) R$ & $(1-\lambda)$ \\
D.6-3 & $\checkmark$ & $p^{2} \sqrt{1-2 p q}$ & $(p q-1) \sqrt{1-2 p q}$ & $q^{2} \sqrt{1-2 p q}$ & \\
D.6-4 & $\times$ & 0 & $\frac{1+p q}{u}$ & 0 & \\
\hline III.6-1 & $\times$ & $\frac{p}{x-q}$ & 0 & 0 & \\
III.6-2 & $\times$ & $2 q(2 p-q u)$ & $q^{2}$ & 0 & \\
\hline
\end{tabular}

The functions $\mathcal{F}_{\mu}$ and $\mathcal{G}_{\kappa}$ are defined as follows:

$$
\begin{aligned}
& \mathcal{F}_{\mu}(z)= \begin{cases}z^{\mu}, & \mu \in \mathbb{C} \backslash\{0,1\} \\
\ln (z), & \mu=0 \\
z \ln (z), & \mu=1 \\
\exp (z), & \mu=\infty\end{cases} \\
& \mathcal{G}_{\kappa}(z)= \begin{cases}z^{\kappa}, & \kappa \in \mathbb{C} \\
\exp (z), & \kappa=\infty\end{cases}
\end{aligned}
$$

In particular, the parameters $\mu, \kappa$ are both allowed to take the value $\infty$ if the contrary is not stated.

Remark 1.3. A checkmark or cross under the SD column indicates that every element in the indicated family is self-dual or not self-dual, respectively. The situation for N.6-2 is more complicated. The corresponding ILC structure is self-dual if and only if the parameters $\mu$ and $\kappa$ satisfy $\mu-\kappa-2=0$ or $\mu+\kappa+1=0$ (see Table A.6).

Remark 1.4. Equations from different items in this list correspond to inequivalent ILC structures. However, there are some additional equivalence relations on parameter spaces for multi-parameter equations within the same item. They are indicated in the last column of Table 1.1.

Our labelling abides by the following rules. The first letter (N, D, or III) denotes the type of the invariant binary quartic. The next digit $(6,7$, or 8$)$ refers to the dimension of the symmetry algebra. The final digit is a labelling of the equation within the given subclass. Finally, the case D. $6-3_{\infty}$ is a limit of D.6-3 as the parameter $\lambda$ tends to infinity.

Table 1.2 describes basic algebraic properties of symmetry algebras for obtained models.

The paper is organized as follows. In Section 2 we provide generalities concerning Legendrian contact structures, establish the relationship between ILC structures and compatible complete systems of 2nd order PDEs, discuss the notion of duality, define the (regular, normal) Cartan connection associated with a given ILC structure, and provide explicit formulas for the fundamental (harmonic) part of its curvature. 
TABLE 1.2. Symmetry algebras of multiply transitive ILC structures

\begin{tabular}{|c|c|c|c|}
\hline Model & Derived series (DS) & Nilradical & Comments \\
\hline N.8 & {$[8,6,4,0]$} & $6-\operatorname{dim}, \mathrm{DS}=[6,4,0], \mathrm{LCS}=[6,4,3,1,0]$ & \\
\hline N.7-1 & {$[7,5,2,0]$} & 5-dim, DS $=\mathrm{LCS}=[5,2,0]$ & \\
\hline N.7-2 & {$[7,6,6]$} & 4-dim abelian & $\left(\mathfrak{s o}_{3} \ltimes \mathbb{C}^{3}\right) \times \mathbb{C}$ \\
\hline N.6-1 & $\begin{cases}{[6,5,2,0],} & \mu \neq 0 \\
{[6,4,1,0],} & \mu=0\end{cases}$ & 5 -dim, DS $=[5,2,0], \mathrm{LCS}=[5,2,1,0]$ & \\
\hline N.6-2 & {$[6,4,0]$} & $\left\{\begin{array}{cl}5 \text {-dim, DS }=[5,2,0], & \\
\text { LCS }=[5,2,1,0] & \mu=\kappa=\infty \\
\text { 4-dim abelian, } & \text { otw }\end{array}\right.$ & \\
\hline D.7 & $\begin{cases}{[7,6,6],} & \lambda \neq 0 \\
{[7,6,4,3,3],} & \lambda=0\end{cases}$ & $\begin{cases}\text { 1-dim abelian, } & \lambda \neq 0 \\
\text { 3-dim Heisenberg, } & \lambda=0\end{cases}$ & $\mathfrak{s l}_{2} \times \mathfrak{s l}_{2} \times \mathbb{C}$ \\
\hline D.6-1 & {$[6,6]$} & 1-dim & $\begin{array}{l}\mathfrak{s l}_{2} \ltimes \mathfrak{s}_{3}, \\
\mathfrak{s}_{3} \text { is Heisenberg }\end{array}$ \\
\hline D.6-2 & {$[6,4,1,0]$} & 4-dim, DS $=\operatorname{LCS}=[4,1,0]$ & \\
\hline D.6-3 & {$[6,6]$} & $0-\operatorname{dim}$ & $\overline{\mathfrak{s l}_{2} \times \mathfrak{s l}_{2}}$ \\
\hline D. $6-3_{\infty}$ & {$[6,6]$} & 3-dim abelian & $\mathfrak{s o}_{3} \ltimes \mathbb{C}^{3}$ \\
\hline D.6-4 & {$[6,6]$} & $0-\operatorname{dim}$ & $\mathfrak{s l}_{2} \times \mathfrak{s l}_{2}$ \\
\hline III.6-1 & {$[6,4,2,0]$} & 4-dim, DS $=[4,2,0], \mathrm{LCS}=[4,2,1,0]$ & \\
\hline III.6-2 & {$[6,5,5]$} & 2-dim abelian & $\mathfrak{g l}_{2} \ltimes \mathbb{C}^{2}$ \\
\hline
\end{tabular}

In Section 3 we specialize to 5-dimensional ILC structures, define the fundamental binary quartic and prove that ILC structures of types I and II cannot be multiply transitive. We also reconstruct the full curvature tensor of the Cartan geometry.

In Section 4 we proceed with the detailed Cartan analysis of the general regular, normal Cartan connection, which involves normalizing parts of the curvature and its derivatives, reducing the Cartan bundle and iterating the procedure. As we are interested only in multiply transitive ILC structures, we terminate this process as soon as the fibers become 0-dimensional. This leads us to the list of all possible structure equations for the reduced bundles. We integrate each of these structure equations and come up with the corresponding ILC model defined in terms of the system of 2nd order PDEs. Finally, in the Appendix we give the detailed Lie algebra isomorphisms establishing the correspondence between the Cartan equations of the reduced bundle and the model systems of 2nd order PDEs, the equivalence relations on the parameters and the duality.

Acknowledgements: The Cartan and DifferentialGeometry packages in Maple (written by Jeanne Clelland and Ian Anderson respectively) provided an invaluable framework for implementing the Cartan reduction method and subsequently carrying out the analysis of the structures obtained. The work of the second and third authors was supported by ARC Discovery grants DP130103485 and DP110100416 respectively. D.T. was also supported by project M1884-N35 of the Austrian Science Fund (FWF). 


\section{LEGENDRIAN CONTACT STRUCTURES}

2.1. Generalities. On any contact manifold $(M, C)$, the contact distribution $C \subset T M$ is locally defined by the vanishing of a 1-form $\sigma$ (unique up to multiplication by a non-vanishing function), and $\left.d \sigma\right|_{C}$ is a (conformal) symplectic form. Given a splitting $C=E \oplus F \subset T M$ into transverse Legendrian subdistributions $E$ and $F$, i.e. $\left.d \sigma\right|_{E}=0$ and $\left.d \sigma\right|_{F}=0$, we say $(M ; E, F)$ is a Legendrian contact $(L C)$ structure. Let $\operatorname{dim}(M)=2 n+1$, so $n=\operatorname{rank}(E)=\operatorname{rank}(F)$. Two LC structures $(M ; E, F)$ and $(\tilde{M} ; \tilde{E}, \tilde{F})$ are (locally) equivalent if there exists a (local) diffeomorphism $\phi: M \rightarrow \tilde{M}$ such that $d \phi(E)=\tilde{E}$ and $d \phi(F)=\tilde{F}$. There is also a natural notion of duality of LC structures: the dual of $(M ; E, F)$ is $(M ; F, E)$.

Since $E$ and $F$ are Legendrian, then $[E, E] \subset C$ and $[F, F] \subset C$. The projections from $C$ onto $E$ and $F$ induce maps $\tau_{E}: \Gamma(E) \times \Gamma(E) \rightarrow \Gamma(F)$ and $\tau_{F}: \Gamma(F) \times \Gamma(F) \rightarrow \Gamma(E)$ that obstruct the integrability of $E$ and $F$. The structure is semi-integrable or integrable according to whether one or both of $\tau_{E}, \tau_{F}$ are identically zero. In the latter case, we call it an $I L C$ structure.

Proposition 2.1. Given any contact manifold $(M, C)$ of dimension $2 n+1$ and a rank $n$ integrable subdistribution $V \subset C$, we may choose local coordinates $\left(x^{i}, u, p_{i}\right)$ on $M$ such that contact form is $\sigma=d u-p_{i} d x^{i}$ and $V=\operatorname{span}\left\{\partial_{p_{i}}\right\}$.

Proof. Since $V$ is integrable and rank $n$, then by the Frobenius theorem there exist local coordinates $\left\{x^{i}\right\}_{i=1}^{2 n+1}$ such that $V=\operatorname{ker}\left\{d x^{1}=\ldots=d x^{n+1}\right\}$. Hence, $C=\operatorname{ker}\{\sigma\}$, where $\sigma=\lambda_{1} d x^{1}+\ldots+$ $\lambda_{n+1} d x^{n+1}$. The contact condition $(d \sigma)^{n} \wedge \sigma \neq 0$ implies that not all $\lambda_{i}$ can simultaneously vanish, so WLOG $\lambda_{n+1} \neq 0$ locally, and after rescaling we may assume $\lambda_{n+1}=1$. Now define $u=x^{n+1}$ and $p_{i}=\lambda_{i}$. The contact condition guarantees that $\left(x^{i}, u, p_{i}\right)$ is indeed a coordinate system.

Suppose that $V:=F$ is integrable, i.e. the LC structure is semi-integrable. By Proposition 2.1, there exist functions $f_{i j}=f_{i j}\left(x^{k}, u, p_{\ell}\right)$ with $f_{i j}=f_{j i}$ (since $E$ is Legendrian) such that

$$
E=\operatorname{span}\left\{\mathcal{D}_{i}:=\partial_{x^{i}}+p_{i} \partial_{u}+f_{i j} \partial_{p_{j}}\right\}, \quad V=\operatorname{span}\left\{\partial_{p_{i}}\right\}
$$

Equivalently, we are studying the geometry of the system of scalar 2nd order PDE

$$
\frac{\partial^{2} u}{\partial x^{i} \partial x^{j}}=f_{i j}(x, u, \partial u), \quad 1 \leq i, j \leq n
$$

considered up to point transformations. These are contact transformations that preserve the (vertical) bundle $V$. All such transformations are precisely the prolongations of arbitrary diffeomorphisms in the $\left(x^{i}, u\right)$ variables. The system (2.2) is overdetermined if $n>1$. If $n=1$, then (2.2) is a single 2nd order ODE, whose point geometry has been well-studied [17].

Remark 2.2. Consider the jet spaces $J^{k}=J^{k}\left(\mathbb{C}^{n}, \mathbb{C}\right)$ and projections $\pi_{\ell}^{k}: J^{k} \rightarrow J^{\ell}$. On $J^{2}$, the contact system is $\left\{d u-p_{i} d x^{i}, d p_{i}-p_{i j} d x^{j}\right\}$, expressed in standard jet coordinates. Pulling back to a submanifold $\mathcal{E}$ defined by $p_{i j}=f_{i j}\left(x^{k}, u, p_{\ell}\right)$ yields the subbundle $E$ in (2.1). The restriction $\left.\pi_{1}^{2}\right|_{\mathcal{E}}: \mathcal{E} \rightarrow J^{1}$ is a local diffeomorphism. The subbundle $V$ in (2.1) is tangent to the fibers of $\pi_{0}^{1} \circ \pi_{1}^{2}$.

Lemma 2.3. The PDE system (2.2) is compatible if and only if $E$ in (2.1) is integrable.

Proof. It is easy to see that $\left[\mathcal{D}_{i}, \mathcal{D}_{j}\right] \in E$ if and only if $\left[\mathcal{D}_{i}, \mathcal{D}_{j}\right]=0$, which happens if and only if $\mathcal{D}_{j} f_{i k}=\mathcal{D}_{i} f_{j k}$ for $1 \leq i, j, k \leq n$. This is exactly the compatibility condition of (2.2).

2.2. Duality. If the dual LC structures $(M ; E, F)$ and $(M ; F, E)$ are equivalent, then we say that the structure is self-dual. For ILC structures, the notion of duality generalizes the classical duality for 2nd order ODE [6]. Namely, for the ILC structure $(M ; E, V)$ given by (2.1), we can (by Proposition 2.1) find coordinates $\left(y^{i}, v, q_{i}\right)$ for the dual ILC structure $(M ; V, E)$, i.e.

$$
V=\operatorname{span}\left\{\partial_{y^{i}}+q_{i} \partial_{v}+\tilde{f}_{i j} \partial_{q_{j}}\right\}, \quad E=\operatorname{span}\left\{\partial_{q_{i}}\right\}
$$


Then $\frac{\partial^{2} v}{\partial x^{i} \partial x^{j}}=\tilde{f}_{i j}$ is the dual system to (2.2) (and is well-defined only up to point transformations).

Example 2.4. The simplest example of an ILC structure is the flat model $u_{i j}=0$. The Legendre transformation $\left(y^{i}, v, q_{k}\right)=\left(p_{i}, u-p_{j} x^{j},-x^{k}\right)$, is a contact (but non-point) transformation which swaps the $E$ and $V$ subbundles, so this structure is self-dual.

Example 2.5. For ILC structures when $n=2$, we have the self-dual D.7 systems:

$$
\mathcal{S}_{\lambda}: \quad u_{11}=p^{2}, \quad u_{12}=0, \quad u_{22}=\lambda q^{2}, \quad \lambda \in \mathbb{C} \backslash\{-1\},
$$

where $p=u_{1}$ and $q=u_{2}$. For fixed $\lambda$, a self-duality, i.e. a swap $(E, V) \mapsto(V, E)$, is exhibited by

$$
\Phi(x, y, u, p, q)= \begin{cases}\left(-\lambda\left(x+\frac{1}{p}\right),-\left(y+\frac{1}{\lambda q}\right),-u+\ln (-p)+\frac{1}{\lambda} \ln (-q), \frac{p}{\lambda}, q\right), & \lambda \neq 0 \\ \left(-\left(x+\frac{1}{p}\right),-q,-u+q y+\ln (-p), p,-y\right), & \lambda=0\end{cases}
$$

Moreover, $\mathcal{S}_{\lambda} \cong \mathcal{S}_{1 / \lambda}$ when $\lambda \neq 0$ via the transformation $\Phi(x, y, u, p, q)=(y, x, \lambda u, \lambda q, \lambda p)$.

As in the case of dual 2nd order ODEs, the dual ILC structures can be constructed in terms of the corresponding PDE models via swapping the space of independent and dependent variables with the space of integration constants parametrizing solutions of a given compatible PDE. In more detail, the general solution of any compatible system (2.2) is parametrized by $n+1$ constants of integration and can be written as:

$$
F\left(x^{i}, u ; a^{j}, b\right)=0, \quad 1 \leq i, j \leq n .
$$

We can consider this as an $(n+1)$-parameter family of hypersurfaces in $\left(x^{i}, u\right)$-space with parameter space $\left(a^{j}, b\right)$. On the other hand, we can (locally) regard $b$ as a function of $a^{j}$, so that (2.3) can be interpreted as an $(n+1)$-parameter family of hypersurfaces in $\left(a^{j}, b\right)$-space with parameter space $\left(x^{i}, u\right)$. This is the solution space of a well-defined compatible system of 2nd order PDE's on $b\left(a^{j}\right)$.

Algorithmically, we construct the dual PDE system by differentiating (2.3) with respect to $a^{j}$ (regarding $x^{i}, u$ as constants and $b$ as a function of $a^{j}$ ), solving the obtained system of $n+1$ equations with respect to $x^{i}, u$ and substituting the solution into the second order derivatives of (2.3) with respect to $a^{j}$.

Example 2.6. In the simplest example of the flat equation $u_{i j}=0$ the general solution is given by:

$$
u=a^{1} x^{1}+\ldots a^{n} x^{n}+b .
$$

Treating $b$ as a function of $a^{j}$, differentiating this solution twice and excluding $x^{i}, u$ we get the same flat equation $b_{i j}=0$. This again demonstrates the self-duality of the flat model.

Example 2.7. The III.6-1 system $u_{11}=\frac{p}{x-q}, u_{12}=u_{22}=0$ has general solution

$$
u=-a y+c-b(x+a)^{2}, \quad a, b, c \in \mathbb{C} .
$$

Regarding $c$ as a function of $a, b$ and treating $x, y, u$ as parameters, we have $c_{a}=y+2 b(x+a)$, $c_{b}=(x+a)^{2}$, and

$$
c_{a a}=2 b, \quad c_{a b}=2(x+a)= \pm 2 \sqrt{c_{b}}, \quad c_{b b}=0 .
$$

WLOG, the \pm ambiguity can be eliminated: the corresponding PDE systems are equivalent via the point transformation $(a, b, c) \mapsto(-a, b, c)$. Thus, the dual system to III.6-1 is

$$
u_{11}=2 y, \quad u_{12}=2 \sqrt{q}, \quad u_{22}=0 .
$$

Our classification indicates that III.6-1 is not self-dual (but a priori this is not at all obvious). 
2.3. LC structures as parabolic geometries. There is an equivalence of categories between (holomorphic) LC structures $(M ; E, F)$ and (regular, normal) parabolic geometries $(\mathcal{G} \rightarrow M, \omega)$ of a fixed type $(G, P)[4$. Here, $G=\operatorname{PGL}(n+2, \mathbb{C})$ acts on the flag variety of pairs of incident lines and hyperplanes:

$$
G / P \cong \operatorname{Flag}_{1, n+1}\left(\mathbb{C}^{n+2}\right)=\{(\ell, \pi): \pi(\ell)=0\} \subset \mathbb{C P}^{n+1} \times\left(\mathbb{C P}^{n+1}\right)^{*},
$$

and $P \subset G$ is the parabolic subgroup which is the stabilizer of a chosen origin. Since $A \in \mathrm{GL}(n+$ $2, \mathbb{C}$ ) and $\lambda A$ (for $\left.\lambda \in \mathbb{C}^{\times}\right)$have the same action on $G / P$, we will instead use $G=\operatorname{SL}(n+2, \mathbb{C})$. The kernel of this action is isomorphic to the cyclic group $\mathbb{Z}_{n+2}$, generated by multiples of the identity matrix by $(n+2)$-th roots of unity. In terms of Lie algebras, $P$ corresponds to the parabolic subalgebra $\mathfrak{p} \subset \mathfrak{g}=\mathfrak{s l}_{n+2}$ defined by the contact grading:

$$
\left.\mathfrak{s l}_{n+2}=\left\{\left[\begin{array}{ccc}
a & U & \gamma \\
X & A & W \\
\beta & Y & b
\end{array}\right]: \begin{array}{l}
b=-a-\operatorname{tr}(A), \\
\text { etc. }
\end{array}\right\}=\mathfrak{C}_{, A \in \mathfrak{g l}_{n},}\right\}=\mathfrak{g}_{-2} \oplus \mathfrak{g}_{-1} \oplus \overbrace{\mathfrak{g}_{0} \oplus \underbrace{\mathfrak{g}_{1} \oplus \mathfrak{g}_{2}}_{\mathfrak{p}_{+}}}^{\mathfrak{p}} .
$$

The reductive part $G_{0} \subset P$ has corresponding subalgebra $\mathfrak{g}_{0} \cong \mathbb{C}^{2} \times \mathfrak{s l}_{n}$ (corresponding to the diagonal blocks $(a, A, b))$ and there is a unique element $Z \in \mathcal{Z}\left(\mathfrak{g}_{0}\right)$ that induces the grading. We refer to the eigenvalues of $Z$ on a particular $\mathfrak{g}_{0}$-module as its homogeneities.

At the origin $o \in G / P$, we have $T_{o}(G / P) \cong \mathfrak{g} / \mathfrak{p}$. Define the subspaces $E_{o}, F_{o} \subset T_{o}(G / P)$ (or subspaces in $\left.\mathfrak{g}_{-1} / \mathfrak{p}\right)$ corresponding to $X, Y$ in (2.4) respectively. The induced $G$-invariant structure $(G / P ; E, F)$ is the flat LC structure, and $\left(G \rightarrow G / P, \omega_{G}\right)$ is the flat model, where $\omega_{G}$ is the MaurerCartan form on $G$. The dimension of the Lie algebra of (infinitesimal) symmetries of the flat model is $\operatorname{dim}(G)=n^{2}+4 n+3$.

A Cartan geometry $(\mathcal{G} \rightarrow M, \omega)$ of type $(G, P)$ is a curved analogue of the flat model. It consists of a principal $P$-bundle $\mathcal{G} \rightarrow M$ equipped with a Cartan connection $\omega \in \Omega^{1}(\mathcal{G} ; \mathfrak{g})$. This means:

(CC.1) $\omega_{u}: T_{u} \mathcal{G} \rightarrow \mathfrak{g}$ is a linear isomorphism for any $u \in \mathcal{G}$;

(CC.2) $R_{p}^{*} \omega=\operatorname{Ad}_{p^{-1}} \circ \omega$ for any $p \in P$;

(CC.3) $\omega\left(\zeta_{A}\right)=A$ for any $A \in \mathfrak{p}$, where $\zeta_{A}(u)=\left.\frac{d}{d t}\right|_{t=0} R_{\exp (t A)}(u)$, i.e. $\zeta_{A}$ is the fundamental vertical vector field corresponding to $A$.

The curvature of $(\mathcal{G} \rightarrow M, \omega)$ is the 2 -form $K=d \omega+\frac{1}{2}[\omega, \omega] \in \Omega^{2}(\mathcal{G} ; \mathfrak{g})$. Using the framing of $T \mathcal{G}$ provided by $\omega$ yields a $P$-equivariant function $\kappa: \mathcal{G} \rightarrow \bigwedge^{2} \mathfrak{g}^{*} \otimes \mathfrak{g}$ which descends to $\kappa$ : $\mathcal{G} \rightarrow \bigwedge^{2}(\mathfrak{g} / \mathfrak{p})^{*} \otimes \mathfrak{g}$ since $K$ is horizontal. For parabolic geometries, the Killing form on $\mathfrak{g}$ yields a $P$-module isomorphism $(\mathfrak{g} / \mathfrak{p})^{*} \cong \mathfrak{p}_{+}$, so we obtain a function $\kappa: \mathcal{G} \rightarrow \bigwedge^{2} \mathfrak{p}_{+} \otimes \mathfrak{g}$. The geometry is

- regular if $\kappa$ is valued in the subspace of $\bigwedge^{2} \mathfrak{p}_{+} \otimes \mathfrak{g}$ consisting of positive homogeneities;

- normal if $\partial^{*} \kappa=0$, where $\partial^{*}$ is the Lie algebra homology differential.

2.4. Harmonic curvature. For regular, normal parabolic geometries, since $\left(\partial^{*}\right)^{2}=0$, we may quotient $\kappa$ by $\operatorname{im}\left(\partial^{*}\right)$ to obtain $\kappa_{H}: \mathcal{G} \rightarrow \frac{\operatorname{ker}\left(\partial^{*}\right)}{\operatorname{im}\left(\partial^{*}\right)}$. This fundamental curvature quantity is called harmonic curvature and is a complete obstruction to flatness of the geometry. The $P$-module $\frac{\operatorname{ker}\left(\partial^{*}\right)}{\operatorname{im}\left(\partial^{*}\right)}$ is completely reducible, so $\mathfrak{p}_{+}$acts trivially. By a result of Kostant [11, 4], the 2-cochains $C^{2}\left(\mathfrak{g}_{-}, \mathfrak{g}\right)$ admit the (orthogonal) $\mathfrak{g}_{0}$-module decomposition

$$
C^{2}\left(\mathfrak{g}_{-}, \mathfrak{g}\right)=\overbrace{\operatorname{im}\left(\partial^{*}\right) \oplus \underbrace{\operatorname{ker}\left(\partial^{*}\right)}_{\operatorname{ker}(\partial)} \oplus \operatorname{im}(\partial)}^{\operatorname{ker}(\square)},
$$

where $\partial$ is the Lie algebra differential, and $\square=\partial \partial^{*}+\partial^{*} \partial$ is the Kostant Laplacian. Thus,

$$
\frac{\operatorname{ker}\left(\partial^{*}\right)}{\operatorname{im}\left(\partial^{*}\right)} \cong \operatorname{ker}(\square) \cong \frac{\operatorname{ker}(\partial)}{\operatorname{im}(\partial)}=: H^{2}\left(\mathfrak{g}_{-}, \mathfrak{g}\right)
$$


The $\mathfrak{g}_{0}$-module structure of the Lie algebra cohomology group $H^{2}\left(\mathfrak{g}_{-}, \mathfrak{g}\right)$ is completely described by Kostant's Bott-Borel-Weil theorem [11, 1, 4]. For LC structures with $n \geq 2, H^{2}\left(\mathfrak{g}_{-}, \mathfrak{g}\right)$ decomposes into three $\mathfrak{g}_{0}$-irreps

$$
H^{2}\left(\mathfrak{g}_{-}, \mathfrak{g}\right)=\mathbb{W} \oplus \mathbb{T}_{1} \oplus \mathbb{T}_{2}
$$

having homogeneities $+2,+1,+1$ respectively. The $\mathbb{T}_{1}$ and $\mathbb{T}_{2}$ components of $\kappa_{H}$ are precisely the torsions $\tau_{E}$ and $\tau_{F}$ (see Section 2.1), and these vanish in the ILC case. Results from twistor theory (see [3]) indicate that the LC structures with trivial $\mathbb{W}$ and $\mathbb{T}_{2}$ components for $\kappa_{H}$ correspond to projective structures. This is the case that was studied by Takeuchi [16].

2.5. Parametric computations of harmonic curvature. Consider a semi-integrable LC structure $(M ; E, V)$ given by (2.1). We will give an explicit formula for the $\mathbb{W}$-component of $\kappa_{H}$.

We use the following co-frame for computations on the manifold $M$ :

$$
\theta^{i}=d x^{i}, \quad \pi_{i}=d p_{i}-f_{i j} d x^{j}, \quad \sigma=d u-p_{i} d x^{i}, \quad 1 \leq i, j \leq n,
$$

so that

$$
E=\operatorname{ker}\left\{\sigma, \pi_{i}\right\}, \quad V=\operatorname{ker}\left\{\sigma, \theta^{i}\right\}
$$

The differential of an arbitrary function $F$ is defined by the formula:

$$
d F=\frac{d F}{d x^{i}} \theta^{i}+\frac{\partial F}{\partial p_{i}} \pi_{i}+\frac{\partial F}{\partial u} \sigma
$$

where $\frac{d}{d x^{i}}:=\mathcal{D}_{i}($ see $(2.1))$ is the total derivative with respect to $x^{i}$.

Let $(\mathcal{G}, \omega)$ be any regular Cartan geometry of type $(G, P)$ with underlying structure $(M ; E, V)$ and curvature $K$. Let $E_{a}{ }^{b} \in \mathfrak{g l}_{n+2}$ denote the element with 1 in the $a$-th row and $b$-column and 0 otherwise. Here, we let $0 \leq a, b \leq n+1$. If $s: M \rightarrow \mathcal{G}$ is any (local) section, write

$$
s^{*} \omega=\omega_{b}^{a} E_{a}^{b}, \quad s^{*} K=K_{b}^{a} E_{a}{ }^{b}
$$

where $K_{b}^{a}=d \omega^{a}{ }_{b}+\omega^{a}{ }_{c} \wedge \omega_{b}^{c}$.

Lemma 2.8. There exists a section $s: M \rightarrow \mathcal{G}$ such that $s^{*} \omega$ satisfies

$$
\omega^{n+1}{ }_{0}=\sigma, \quad \omega_{0}^{i}=\theta^{i}, \quad \omega^{n+1}{ }_{i}=\pi_{i}, \quad \omega_{0}^{0} \equiv 0 \bmod \left\{\theta^{i}, \pi_{i}\right\}
$$

Proof. Consider a section $s: M \rightarrow \mathcal{G}$. Since $\omega$ is regular, the negative part of $s^{*} \omega$ is an adapted coframe, i.e.

$$
\omega_{0}^{n+1}=e \sigma, \quad \omega^{n+1}{ }_{i}=g_{i}^{j} \pi_{j}+g_{i} \sigma, \quad \omega_{0}^{i}=h_{j}^{i} \theta^{j}+h^{i} \sigma .
$$

An arbitrary section $\tilde{s}$ is given in terms of a function $h: M \rightarrow P$ such that $\tilde{s}=s \cdot h$. This satisfies:

$$
\tilde{s}^{*} \omega=h^{-1}\left(s^{*} \omega\right) h+h^{-1} d h .
$$

Since $h^{-1} d h$ term is $\mathfrak{p}$-valued, the negative part of $s^{*} \omega$ transforms via the adjoint action.

Using the $G_{0}$-action, we can normalize $e=1$ and $g^{j}{ }_{i}=\delta^{j}{ }_{i}$. Since

$$
K_{0}^{n+1}=d \omega_{0}^{n+1}+\omega^{n+1}{ }_{a} \wedge \omega_{0}^{a} \equiv d \sigma+\omega^{n+1}{ }_{i} \wedge \omega_{0}^{i} \equiv\left(-\pi_{i}+h^{j}{ }_{i} \pi_{j}\right) \wedge \theta^{i} \bmod \sigma,
$$

and regularity implies $K^{n+1}{ }_{0} \equiv 0 \bmod \sigma$, then $h^{i}{ }_{j}=\delta^{i}{ }_{j}$. Using the action of subgroup of $P$ corresponding to $\mathfrak{g}_{1}$, we can normalize $g_{i}=0, h^{i}=0$. Similarly, using the subgroup of $P$ corresponding to $\mathfrak{g}_{2}$, we can normalize $\omega_{0}^{0} \equiv 0 \bmod \left\{\theta^{i}, \pi_{j}\right\}$.

With respect to such a section, write

$$
\omega_{b}^{a}=r_{b i}^{a} \theta^{i}+s_{b}^{a}{ }^{i} \pi_{i}+t^{a}{ }_{b} \sigma .
$$

To obtain the harmonic part of the normal curvature, it is sufficient to compute normalization conditions only in homogeneities 1 and 2. For any regular, normal parabolic geometry, the lowest homogeneity curvature component is harmonic [4]. Thus, all curvature components in homogeneity 
1 must vanish except the coefficients of $\theta^{j} \wedge \theta^{k}$ in $K^{n+1}{ }_{i}$, and this corresponds to the torsion of our semi-integrable structure. Recalling that $\omega^{n+1}{ }_{n+1}=-\omega^{0}{ }_{0}-\omega^{i}{ }_{i}$ since $\omega$ is $\mathfrak{s l}_{n+2}$-valued, we have:

$$
\begin{aligned}
& K_{0}^{n+1}=d \omega^{n+1}{ }_{0}+\omega^{n+1}{ }_{a} \wedge \omega_{0}^{a}=\omega^{n+1}{ }_{0} \wedge \omega_{0}^{0}+\omega^{n+1}{ }_{n+1} \wedge \omega^{n+1}{ }_{0}=\sigma \wedge\left(2 \omega_{0}^{0}+\omega_{i}^{i}\right) \\
& =\left(2 r^{0}{ }_{0 j}+r^{i}{ }_{i j}\right) \sigma \wedge \theta^{j}+\left(2 s^{0}{ }_{0}{ }^{j}+s^{i}{ }_{i}{ }^{j}\right) \sigma \wedge \pi_{j} \\
& K_{0}^{i}=d \omega_{0}^{i}+\omega^{i}{ }_{a} \wedge \omega^{a}{ }_{0} \equiv \omega_{0}^{i} \wedge \omega_{0}^{0}+\omega^{i}{ }_{j} \wedge \omega^{j}{ }_{0} \quad \bmod \sigma \\
& \equiv\left(r^{i}{ }_{[j k]}+r^{0}{ }_{0[j} \delta_{k]}^{i}\right) \theta^{k} \wedge \theta^{j}+\left(s^{i}{ }_{j}{ }^{k}-s^{0}{ }_{0}{ }^{k} \delta^{i}{ }_{j}\right) \pi_{k} \wedge \theta^{j} \bmod \sigma \\
& K_{i}^{n+1}=d \omega^{n+1}{ }_{i}+\omega^{n+1}{ }_{a} \wedge \omega^{a}{ }_{i} \equiv d \pi_{i}+\pi_{j} \wedge \omega^{j}{ }_{i}+\omega^{n+1}{ }_{n+1} \wedge \pi_{i} \quad \bmod \sigma \\
& \equiv \frac{d f_{i j}}{d x^{k}} \theta^{j} \wedge \theta^{k}+\left(r^{j}{ }_{i k}+\left(r_{0 k}^{0}+r_{l k}^{l}\right) \delta_{i}^{j}-\frac{\partial f_{i k}}{\partial p_{j}}\right) \pi_{j} \wedge \theta^{k} \\
& +\left(s_{i}^{j}{ }^{k}+\left(s^{0}{ }_{0}{ }^{k}+s^{l}{ }_{l}^{k}\right) \delta^{j}{ }_{i}\right) \pi_{j} \wedge \pi_{k} \bmod \sigma
\end{aligned}
$$

We confirm that the coefficient of $\theta^{j} \wedge \theta^{k}$ in $K^{n+1}{ }_{i}$ is indeed the obstruction $\mathcal{D}_{k} f_{i j}-\mathcal{D}_{j} f_{i k}$ to integrability of $E$. All remaining terms above are zero, so we get:

$$
s_{i}^{j}{ }_{i}^{k}=0, \quad s_{0}^{0}{ }^{i}=0, \quad r_{0 i}^{0}=-\frac{1}{n+2} \frac{\partial f_{i j}}{\partial p_{j}}, \quad r_{j k}^{i}=\frac{\partial f_{j k}}{\partial p_{i}}-\delta^{i}{ }_{j} \frac{1}{n+2} \frac{\partial f_{l k}}{\partial p_{l}} .
$$

Proceed now to homogeneity 2. Using (2.6), we compute:

$$
\begin{aligned}
K_{0}^{i}= & s^{i}{ }_{n+1}{ }^{j} \pi_{j} \wedge \sigma+\left(r^{i}{ }_{n+1, j}-t^{i}{ }_{j}\right) \theta^{j} \wedge \sigma \\
K^{n+1}{ }_{i}= & \frac{d f_{i j}}{d x^{k}} \theta^{j} \wedge \theta^{k}+\left(\frac{\partial f_{i j}}{\partial u}-r^{0}{ }_{i j}\right) \theta^{j} \wedge \sigma+\left(t^{j}{ }_{i}-s^{0}{ }_{i}{ }^{j}+\delta_{i}^{j} t^{k}{ }_{k}\right) \pi_{j} \wedge \sigma \\
K_{0}^{0} \equiv & \left(\frac{d r^{0}{ }_{0 i}}{d x^{j}}+r^{0}{ }_{i j}\right) \theta^{j} \wedge \theta^{i}+\left(\frac{\partial r^{0}{ }_{0 i}}{\partial p_{j}}+s^{0}{ }_{i}{ }^{j}\right) \pi_{j} \wedge \theta^{i} \bmod \sigma \\
K^{i}{ }_{j} \equiv & \left(\frac{d r^{i}{ }_{j l}}{d x^{k}}+\delta^{i}{ }_{k} r^{0}{ }_{j l}+r^{i}{ }_{p k} r^{p}{ }_{j l}\right) \theta^{k} \wedge \theta^{l}+\left(\frac{\partial r^{i}{ }_{j l}}{\partial p_{k}}-\delta^{k}{ }_{l} t^{i}{ }_{j}-\delta^{k}{ }_{j} r^{i}{ }_{n+1, l}-\delta^{i}{ }_{l} s^{0}{ }_{j}{ }^{k}\right) \pi_{k} \wedge \theta^{l} \\
& \quad+s^{i}{ }_{n+1}{ }^{k} \pi_{k} \wedge \pi_{j} \bmod \sigma
\end{aligned}
$$

To obtain the pullback $s^{*} \kappa: M \rightarrow \bigwedge^{2} \mathfrak{p}_{+} \otimes \mathfrak{g}$ of the curvature function $\kappa: \mathcal{G} \rightarrow \bigwedge^{2} \mathfrak{p}_{+} \otimes \mathfrak{g}$, we note that the framing provided by $\omega$ together with $P$-equivariancy of $\kappa$ allows us to identify $\sigma=\omega^{n+1}{ }_{0}, \theta^{i}=\omega_{0}^{i}$ and $\pi_{i}=\omega^{n+1}{ }_{i}$ with $\left(E_{j}{ }^{0}\right)^{*},\left(E_{n+1}{ }^{j}\right)^{*}$ and $\left(E_{0}{ }^{n+1}\right)^{*}$ respectively. A form $B$ on $\mathfrak{g l}_{n+2}$ which is defined by $B(X, Y)=\operatorname{tr}(X Y)$ and is proportional to the Killing form on $\mathfrak{g l}_{n+2}$ induces a $P$-module isomorphism $(\mathfrak{g} / \mathfrak{p})^{*} \cong \mathfrak{p}_{+}$. This allows us to make the replacements

$$
\theta^{j} \leftrightarrow E_{0}^{j}, \quad \pi_{j} \leftrightarrow E_{j}^{n+1}, \quad \sigma \leftrightarrow E_{0}^{n+1}
$$

in the curvature 2 -form $K$. The homology differential $\partial^{*}: \bigwedge^{2} \mathfrak{p}_{+} \otimes \mathfrak{g} \rightarrow \mathfrak{p}_{+} \otimes \mathfrak{g}$ is defined on decomposable elements as

$$
\partial^{*}(X \wedge Y \otimes v)=-Y \otimes[X, v]+X \otimes[Y, v]-[X, Y] \otimes v .
$$

We introduce a bi-grading on $\bigwedge^{\bullet} \mathfrak{p}_{+} \otimes \mathfrak{g}$. Let $\mathfrak{h} \subset \mathfrak{g l}_{n+2}$ be Cartan subalgebra for the standard upper-triangular Borel subalgebra. Let also $Z_{i} \in \mathfrak{h}, 1 \leq i \leq n+1$ be a dual basis to the simple roots basis $\alpha_{i} \in \mathfrak{h}^{*}, 1 \leq i \leq n+1$. Then the pair $\left(Z_{1}, Z_{n+1}\right)$ induces bi-grading $X \rightarrow\left(a_{1}, a_{n+1}\right)$ where $\left[Z_{i}, X\right]=a_{i} X$ for $i=1, n+1$. Homogeneity of an element $X$ is equal to $a_{1}+a_{n+1}$ since $Z=Z_{1}+Z_{n+1}$ where $Z$ is a grading element. Moreover, since $\partial^{*}$ is $P$-equivariant map it respects bi-grading.

In order to compute harmonic curvature it is sufficient to use only $\partial^{*} \kappa_{(1,1)}=0$ and $\partial^{*} \kappa_{(0,2)}=0$ normality conditions. Using (2.7)-(2.10) and $K^{n+1}{ }_{n+1}=-K_{0}^{0}-K_{i}^{i}$ we compute: 


$$
\begin{aligned}
& 0=\partial^{*} \kappa_{(1,1)}=\left(r^{i}{ }_{n+1, j}-t^{i}{ }_{j}\right)\left(E_{0}{ }^{n+1} \otimes\left(E_{i}{ }^{j}-\delta_{i}{ }^{j} E_{0}{ }^{0}\right)-E_{0}{ }^{j} \otimes E_{i}{ }^{n+1}\right) \\
& +\left(t^{j}{ }_{i}-s^{0}{ }_{i}{ }^{j}+\delta^{j}{ }_{i} t^{k}{ }_{k}\right)\left(-E_{0}{ }^{n+1} \otimes\left(E_{j}{ }^{i}-\delta_{j}{ }^{i} E_{n+1}{ }^{n+1}\right)+E_{j}{ }^{n+1} \otimes E_{0}{ }^{i}\right) \\
& +\left(\frac{\partial r^{0}{ }_{0 i}}{\partial p_{j}}+s^{0}{ }_{i}{ }^{j}\right)\left(-E_{j}{ }^{n+1} \otimes E_{0}{ }^{i}+\delta^{i}{ }_{j} E_{0}{ }^{n+1} \otimes E_{0}{ }^{0}\right) \\
& +\left(\frac{\partial r^{i}{ }_{j l}}{\partial p_{k}}-\delta^{k}{ }_{l} t^{i}{ }_{j}-\delta^{k}{ }_{j} r^{i}{ }_{n+1, l}-\delta^{i}{ }_{l} s^{0}{ }_{j}{ }^{k}\right)\left(E_{0}{ }^{l} \otimes \delta^{j}{ }_{k} E_{i}{ }^{n+1}+E_{k}{ }^{n+1} \otimes \delta^{l}{ }_{i} E_{0}{ }^{j}+\delta^{l}{ }_{k} E_{0}{ }^{n+1} \otimes E_{i}{ }^{j}\right) \\
& +\left(\frac{\partial r_{0 l}^{0}}{\partial p_{k}}+\frac{\partial r^{i}{ }_{i l}}{\partial p_{k}}-\delta^{k}{ }_{l} t_{i}-r_{n+1, l}^{k}\right)\left(E_{0}{ }^{l} \otimes E_{k}{ }^{n+1}-\delta_{k}{ }^{l} E_{0}{ }^{n+1} \otimes E_{n+1}{ }^{n+1}\right) \\
& =\left(\frac{\partial r^{j}{ }_{k i}}{\partial p_{k}}+\frac{\partial r^{0}{ }_{0 i}}{\partial p_{j}}+\frac{\partial r^{k}{ }_{k i}}{\partial p_{j}}-(n+2) r^{j}{ }_{n+1, i}-\delta^{j}{ }_{i}\left(s^{0}{ }_{k}{ }^{k}+t_{k}^{k}\right)\right) E_{0}{ }^{i} \otimes E_{j}{ }^{n+1} \\
& +\left(\frac{\partial r^{i}{ }_{j k}}{\partial p_{k}}-(n+2) t^{i}{ }_{j}-\delta^{i}{ }_{j} t_{k}{ }_{k}\right) E_{0}{ }^{n+1} \otimes\left(E_{i}{ }^{j}-\delta_{i}{ }^{j} E_{n+1}{ }^{n+1}\right) \\
& +\left(\frac{\partial r_{0 i}^{0}}{\partial p_{i}}+t^{i}{ }_{i}+s^{0}{ }_{i}{ }^{i}-r_{n+1, i}^{i}\right) E_{0}{ }^{n+1} \otimes\left(E_{0}{ }^{0}-E_{n+1}{ }^{n+1}\right) \\
& +\left(\frac{\partial r^{k}{ }_{i k}}{\partial p_{j}}-\frac{\partial r^{0}{ }_{0 i}}{\partial p_{j}}-(n+2) s^{0}{ }_{i}{ }^{j}+\delta^{j}{ }_{i}\left(t^{k}{ }_{k}-r^{k}{ }_{n+1, k}\right)\right) E_{j}{ }^{n+1} \otimes E_{0}{ }^{i}, \\
& 0=\partial^{*} \kappa_{(0,2)}=-s^{i}{ }_{n+1}{ }^{j} E_{j}{ }^{n+1} \otimes E_{i}{ }^{n+1}+s^{i}{ }_{n+1}{ }^{k}(1-n) E_{k}{ }^{n+1} \otimes E_{i}{ }^{n+1}-s^{i}{ }_{n+1}{ }^{j} E_{j}{ }^{n+1} \otimes E_{i}{ }^{n+1} \\
& +s^{i}{ }_{n+1}{ }^{j} E_{i}{ }^{n+1} \otimes E_{j}{ }^{n+1}=\left(s^{j}{ }_{n+1}{ }^{i}-(n+1) s^{i}{ }_{n+1}{ }^{j}\right) E_{j}{ }^{n+1} \otimes E_{i}{ }^{n+1} \text {. }
\end{aligned}
$$

Substituting (2.6) we obtain linear system of equations on coefficients of normal regular Cartan connection:

$$
\begin{aligned}
0 & =\frac{\partial^{2} f_{i k}}{\partial p_{j} \partial p_{k}}-(n+2) r^{j}{ }_{n+1, i}-\delta^{j}{ }_{i}\left(s^{0}{ }_{k}{ }^{k}+t^{k}{ }_{k}\right), \\
0 & =\frac{\partial^{2} f_{j k}}{\partial p_{i} \partial p_{k}}-\delta^{i}{ }_{j} \frac{1}{n+2} \frac{\partial^{2} f_{l k}}{\partial p_{l} \partial p_{k}}-(n+2) t_{j}^{i}-\delta^{i}{ }_{j}, t_{k}^{k} \\
0 & =-\frac{1}{n+2} \frac{\partial^{2} f_{i j}}{\partial p_{i} \partial p_{j}}+t^{i}{ }_{i}+s^{0}{ }_{i}{ }^{i}-r^{i}{ }_{n+1, i}, \\
0 & =\frac{\partial^{2} f_{i k}}{\partial p_{j} \partial p_{k}}-(n+2) s^{0}{ }_{i}{ }^{j}+\delta^{j}{ }_{i}\left(t^{k}{ }_{k}-r^{k}{ }_{n+1, k}\right) .
\end{aligned}
$$

Solving the linear system of equations above we get the homogeneity 2 coefficients of normal Cartan connection needed for the computation of $\kappa_{H}$ :

$$
\begin{aligned}
t^{i}{ }_{j} & =\frac{1}{n+2} \frac{\partial^{2} f_{j k}}{\partial p_{i} \partial p_{k}}-\delta_{j}^{i} \frac{1}{(n+2)(n+1)} \frac{\partial^{2} f_{l k}}{\partial p_{l} \partial p_{k}}, \\
r^{i}{ }_{n+1, j} & =t^{i}{ }_{j}, \\
s_{i}^{0}{ }^{j} & =\frac{1}{n+2} \frac{\partial^{2} f_{i k}}{\partial p_{j} \partial p_{k}}, \\
s_{n+1}{ }_{n+1}{ }^{j} & =0 .
\end{aligned}
$$

From Kostant's theorem we know that $\mathbb{W}$ has the lowest weight vector $\phi_{0}=E_{0}{ }^{1} \wedge E_{n}{ }^{n+1} \otimes E_{n}{ }^{1}$. The element $\phi_{0}$ belongs to the module $\mathbb{V}$ generated by: 


$$
w_{l}^{k}{ }_{i}^{j}=E_{0}{ }^{k} \wedge E_{l}{ }^{n+1} \otimes\left(E_{i}{ }^{j}-\delta_{i}{ }^{j} E_{n+1}{ }^{n+1}\right) .
$$

The module $\mathbb{W}$ is the submodule of $\mathbb{V}$ consisting of tensors that are trace-free in $(i, j)$, symmetric in $(l, i)$, and symmetric in $(k, j)$. We denote coefficients of $\kappa$ which corresponds to $w_{l}{ }^{k}{ }_{i}{ }^{j}$ as $W^{l}{ }_{k}{ }_{j}{ }_{j}$ and assume that

$$
T_{i j}^{k}=-\frac{\partial^{2} f_{i j}}{\partial p_{k} \partial p_{l}} .
$$

and $T^{l}{ }_{j}=T^{i}{ }_{i j}{ }_{j}, T=T^{i}{ }_{i j}{ }_{j}$. According to (2.10)

$$
W_{k j}^{l}{ }^{i}=-\left(\frac{\partial r^{i}{ }_{j k}}{\partial p_{l}}-\delta_{k}^{l} t^{i}{ }_{j}-\delta^{l}{ }_{j} r^{i}{ }_{n+1, k}-\delta^{i}{ }_{k}{ }^{l}{ }_{j,-1}\right) .
$$

Using (2.6) and (2.12)-(2.14) we obtain that $W^{l}{ }_{k}{ }_{j}{ }_{j}$ is equal to trace-free part of tensor $T^{l}{ }_{k}{ }_{j}$ :

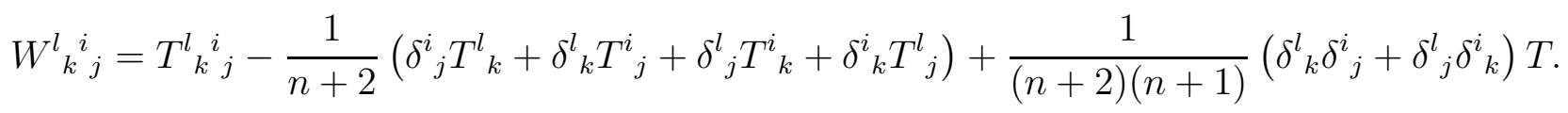

Coefficients $W_{k}^{l}{ }_{k}{ }_{j}$ are symmetric in $(i, l),(j, k)$, trace-free in $(i, j)$ and form the $\mathbb{W}$-component of $\kappa_{H}$.

We summarize computations of this sub-section in the following theorem.

Theorem 2.9. With respect to the section s defined by Lemma 2.8, the $\mathbb{W}$ component of the harmonic curvature of the regular, normal connection for semi-integrable LCS given by 2.1] is

$$
W^{l}{ }_{k j}{ }_{j} E_{0}{ }^{k} \wedge E_{l}{ }^{n+1} \otimes\left(E_{i}{ }^{j}-\delta_{i}{ }^{j} E_{n+1}{ }^{n+1}\right),
$$

where $W_{k}^{l}{ }_{k}{ }_{j}$ is the trace-free part of the tensor

$$
T_{k j}^{l i}=-\frac{\partial^{2} f_{k j}}{\partial p_{l} \partial p_{i}}
$$

\section{ILC STRUCTURES IN DIMENSION FIVE}

Henceforth, we specialize to the $n=2$ ILC case, which corresponds to compatible PDE systems

$$
u_{11}=F, \quad u_{12}=G, \quad u_{22}=H,
$$

where $F, G, H$ are functions of $(x, y, u, p, q)$ with $p=u_{1}$ and $q=u_{2}$. Equivalently, $E$ and $V$ as in (2.1) (with $f_{11}=F, f_{12}=G, f_{22}=H$ ) are both integrable.

Let us fix notation for $\mathfrak{p}$. Take the standard (upper triangular) Borel subalgebra, diagonal Cartan subalgebra $\mathfrak{h} \subset \mathfrak{s l}_{4}$, and simple roots $\alpha_{i}=\epsilon_{i}-\epsilon_{i+1} \in \mathfrak{h}^{*}$ for $i=1,2,3$. The dual basis $Z_{1}, Z_{2}, Z_{3} \in \mathfrak{h}$ to the simple roots is given by

$$
Z_{1}=\operatorname{diag}\left(\frac{3}{4},-\frac{1}{4},-\frac{1}{4},-\frac{1}{4}\right), \quad Z_{2}=\operatorname{diag}\left(\frac{1}{2}, \frac{1}{2},-\frac{1}{2},-\frac{1}{2}\right), \quad Z_{3}=\operatorname{diag}\left(\frac{1}{4}, \frac{1}{4}, \frac{1}{4},-\frac{3}{4}\right) .
$$

The grading element adapted to $P$ is $Z:=Z_{1}+Z_{3}$. Use linear coordinates on $\mathfrak{p}$ :

$$
\left[\begin{array}{cccc}
\frac{3 z_{1}+z_{2}}{4} & t_{1} & t_{2} & t_{5} \\
0 & v_{1}+\frac{z_{2}-z_{1}}{4} & v_{2} & t_{3} \\
0 & v_{3} & -v_{1}+\frac{z_{2}-z_{1}}{4} & t_{4} \\
0 & 0 & 0 & -\frac{z_{1}+3 z_{2}}{4}
\end{array}\right] \in \mathfrak{p}
$$

We have $\mathfrak{g}_{0}=\mathcal{Z}\left(\mathfrak{g}_{0}\right) \times\left(\mathfrak{g}_{0}\right)_{s s} \cong \mathbb{C}^{2} \times \mathfrak{s l}_{2}$, where $\mathbb{C}^{2}=\operatorname{span}\left\{Z_{1}, Z_{3}\right\}$. In terms of the standard basis $\left\{E_{a}{ }^{b}\right\}_{0 \leq a, b \leq 3}$ of $\mathfrak{g l}_{4}$, a standard $\mathfrak{s l}_{2}$-triple spanning the semisimple part $\left(\mathfrak{g}_{0}\right)_{s s} \subset \mathfrak{p}$ is given by:

$$
\mathrm{H}:=E_{1}{ }^{1}-E_{2}{ }^{2}, \quad \mathrm{X}:=E_{1}{ }^{2}, \quad \mathrm{Y}:=E_{2}{ }^{1} .
$$


For ILC structures, $\kappa_{H}$ takes values in ${ }^{2}$ the module $\mathbb{W}=\stackrel{-3}{\times} \stackrel{4}{0}^{-3} \times$ (in the notation of [1]). With respect to $\left(Z_{1}, Z_{3}\right)$, W has bi-grading $(+1,+1)$ so that its homogeneity is +2 . As $\mathfrak{s l}_{2}$-modules, $\mathbb{W} \cong \bigodot^{4}\left(\mathbb{C}^{2}\right)$, i.e. the space of binary quartics in $\mathrm{r}$, s, say. Hence, $\kappa_{H}$ (up to sign) is:

$$
\kappa_{H}=F_{q q} r^{4}+2\left(F_{p q}-G_{q q}\right) r^{3} s+\left(F_{p p}-4 G_{p q}+H_{q q}\right) r^{2} s^{2}+2\left(H_{p q}-G_{p p}\right) \mathrm{rs}^{3}+H_{p p} \mathbf{s}^{4} .
$$

Strictly speaking, this is the pullback of $\kappa_{H}$ by a (local) section $s: M \rightarrow \mathcal{G}$. Since $P_{+}$acts vertically trivially on $\operatorname{ker}\left(\partial^{*}\right) / \operatorname{im}\left(\partial^{*}\right)$, (3.3) is canonically defined only up to a $G_{0}$-transformation.

3.1. Petrov classification. As in the Petrov classification of the Weyl tensor in 4-dimensional Lorentzian (conformal) geometry and the classification of $(2,3,5)$-distributions [5], ILC structures can be classified based on the (pointwise) root type of the binary quartic field (3.3). We use the same notation for types as in the Petrov classification, e.g. type N and D indicate a single quadruple root and a pair of double roots respectively.

Any ILC structure admits at most a 15-dimensional symmetry algebra and 15 is realized only on (an open subset of) the flat model (up to local isomorphism). Among (regular, normal) parabolic geometries $(\mathcal{G} \rightarrow M, \omega)$ of a given type $(G, P)$, Kruglikov and The [12] gave a general method for finding the submaximal symmetry dimension, i.e. the symmetry dimension for any non-flat structure, and for ILC structures this dimension is eight. These techniques can also be used to determine the maximal symmetry dimension for ILC structures with constant root type. We briefly outline their method. A non-trivial root type corresponds to a $G_{0}$-orbit $\{0\} \neq \mathcal{O} \subset \mathbb{W}$ (or in type I, a collection of $G_{0}$-orbits). Defining $\mathfrak{a}^{\phi}=\mathfrak{g}_{-} \oplus \mathfrak{a n n}(\phi)$ for non-flat ILC structures, we have:

$$
\operatorname{dim}(\mathfrak{i n f}(\mathcal{G}, \omega)) \leq \max \left\{\operatorname{dim}\left(\mathfrak{a}^{\phi}\right): \phi \in \mathcal{O}\right\}=5+\max \{\operatorname{dim}(\mathfrak{a n n}(\phi)): \phi \in \mathcal{O}\} .
$$

Since $\operatorname{dim}(\mathfrak{a n n}(\phi))$ is constant along $G_{0}$-orbits, it suffices to evaluate it on a cross-section.

Theorem 3.1. Among ILC structures with constant root type, we have:

\begin{tabular}{|c|c|c|c|c|c|c|}
\hline Root type & $O$ & $N$ & $D$ & $I I I$ & $I I$ & $I$ \\
\hline \hline Max. sym. dim. & 15 & 8 & 7 & 6 & 5 & 5 \\
\hline Sharp? & $\checkmark$ & $\checkmark$ & $\checkmark$ & $\checkmark$ & $\checkmark$ & $\checkmark$ \\
\hline
\end{tabular}

Proof. See Table 1.1 for type N, D, III models with the stated symmetry dimensions.

A Type I model with 5-dimensional symmetry is given by:

$$
u_{11}=6 S^{5 / 3}-6 u S^{4 / 3}+2\left(u^{2}-q\right) S-2 p q, \quad u_{12}=3 S^{4 / 3}-2 u S-q^{2}, \quad u_{22}=2 S,
$$

where $S=p+u q$. Its harmonic curvature is given by the quartic:

$$
\kappa_{H}=-\frac{4}{3} \mathrm{r}(u \mathrm{r}+\mathrm{s})\left(\mathrm{r}-(u \mathrm{r}+\mathrm{s}) S^{-1 / 3}\right)\left(3 \mathrm{r}-2(u \mathrm{r}+\mathrm{s}) S^{-1 / 3}\right),
$$

which has four distinct roots on the open set $\{S \neq 0\}$. The equation is invariant with respect to the action of $\mathfrak{s l}_{2} \ltimes \mathbb{C}^{2}$ generated by:

$$
\begin{array}{rr}
\partial_{x}, \quad \partial_{y}, \quad x \partial_{y}+\partial_{u}-q \partial_{p}, \quad 2 x \partial_{x}+y \partial_{y}-u \partial_{u}-3 p \partial_{p}-2 q \partial_{q}, \\
x^{2} \partial_{x}+x y \partial_{y}+(y-x u) \partial_{u}-(u+3 x p+y q) \partial_{p}+(1-2 x q) \partial_{q} .
\end{array}
$$

Next, consider

$$
u_{11}=p^{\lambda+2} q^{\mu}, \quad u_{12}=p^{\lambda+1} q^{\mu+1}, \quad u_{22}=p^{\lambda} q^{\mu+2},
$$

which is type II when $\lambda, \mu \neq 0, \lambda+\mu \neq 0,1$ according to

$$
\kappa_{H}=p^{\lambda-2} q^{\mu-2}(p \mathrm{r}-q \mathrm{~s})^{2}\left(\mu(\mu-1) p^{2} \mathrm{r}^{2}+2 \lambda \mu p q \mathrm{rs}+\lambda(\lambda-1) q^{2} \mathrm{~s}^{2}\right) .
$$

\footnotetext{
${ }^{2}$ In terms of $\mathfrak{s l}_{4}$ weights $\left\{\lambda_{i}\right\}, \mathbb{W}$ has lowest weight $3 \lambda_{1}-4 \lambda_{2}+3 \lambda_{3}=\alpha_{1}-\alpha_{2}+\alpha_{3}$ by the "minus lowest weight" convention [1].
} 
The symmetry algebra is generated by the 5 vector fields:

$\partial_{x}, \quad \partial_{y}, \quad \partial_{u}, \quad-\mu\left(x \partial_{x}-p \partial_{p}\right)+\lambda\left(y \partial_{y}-q \partial_{q}\right), \quad(1+\lambda+\mu)\left(x \partial_{x}+y \partial_{y}\right)+(\lambda+\mu) u \partial_{u}-p \partial_{p}-q \partial_{q}$.

Now we establish upper bounds. Up to scale, representative elements in the $G_{0}$-orbits are

$$
\mathrm{N}: \mathrm{s}^{4} ; \quad \text { D: } \mathrm{r}^{2} \mathrm{~s}^{2} ; \quad \text { III: } \mathrm{rs}^{3} ; \quad \text { II: } \mathrm{r}^{2} \mathrm{~s}(\mathrm{r}-\mathrm{s}) ; \quad \mathrm{I}: \mathrm{rs}(\mathrm{r}-\mathrm{s})(\mathrm{r}-\mathrm{cs}), \quad c \in \mathbb{C} \backslash\{0,1\} .
$$

The annihilators of the above elements, cf. (3.2), are spanned by:

$$
\mathrm{N}: Z_{1}-Z_{3}, Y, H+4 Z_{1} ; \quad \text { D: } Z_{1}-Z_{3}, H ; \quad \text { III: } Z_{1}-Z_{3}, H+2 Z_{1} ; \quad \text { II, I: } Z_{1}-Z_{3} .
$$

By (3.4), the result is proved for N and D, while for III, II, I the upper bound is one more than in the stated result. For the latter, we show that the upper bound is never realizable.

Consider the type III orbit and assume there is a model with 7-dimensional symmetry algebra s. According to [12, Cor.3.4.8] (in particular, ILC structures are "prolongation-rigid"), $\mathfrak{s}$ admits a natural filtration $\mathfrak{s}=\mathfrak{s}^{(-2)} \supset \mathfrak{s}^{(-1)} \supset \mathfrak{s}^{(0)}$ with associated-graded Lie algebra isomorphic to $\mathfrak{g}_{-2} \oplus \mathfrak{g}_{-1} \oplus \mathfrak{a}_{0}$, where $\mathfrak{a}_{0}$ is the above annihilator of the type III orbit and $\mathfrak{g}_{-1}, \mathfrak{g}_{-2}$ are graded subspaces of $\mathfrak{g}=\mathfrak{s l}_{4}$. In other words, $\mathfrak{s}$ is a filtered deformation of the above 7 -dimensional graded Lie algebra.

Any such deformation is necessarily invariant with respect to $\mathfrak{s}^{(0)}=\mathfrak{a}_{0}$. Fix a basis $T_{1}=Z_{1}-$ $Z_{3}, T_{2}=H+2 Z_{1}$ in $\mathfrak{a}_{0}$. Its action on $\mathfrak{g}_{-1}$ and $\mathfrak{g}_{-2}$ diagonalizes with pairs of eigenvalues $(-1,-1)$, $(-1,-3),(1,-1),(1,1)$ and $(0,-2)$ respectively. Denote by $E_{1}=E_{1}^{0}, E_{2}=E_{2}{ }^{0}, F_{1}=E_{3}{ }^{1}$, $F_{2}=E_{3}{ }^{2}, U=-E_{3}{ }^{0}$ the corresponding eigenvectors of this action. Then all possible deformations of $\mathfrak{a}_{0} \oplus \mathfrak{g}_{-1} \oplus \mathfrak{g}_{-2}$ preserving the filtration and the action of $\mathfrak{a}_{0}$ have the form:

$$
\begin{aligned}
& {\left[T_{1}, E_{1}\right]=-E_{1}, \quad\left[T_{1}, E_{2}\right]=-E_{2}, \quad\left[T_{1}, F_{1}\right]=F_{1}, \quad\left[T_{1}, F_{2}\right]=F_{2},} \\
& {\left[T_{2}, E_{1}\right]=-E_{1}, \quad\left[T_{2}, E_{2}\right]=-3 E_{2}, \quad\left[T_{2}, F_{1}\right]=-F_{1}, \quad\left[T_{2}, F_{2}\right]=F_{2}, \quad\left[T_{2}, U\right]=-2 U,} \\
& {\left[E_{1}, F_{1}\right]=U, \quad\left[E_{1}, F_{2}\right]=a T_{1}+b T_{2}, \quad\left[E_{2}, F_{2}\right]=U, \quad\left[F_{2}, U\right]=c F_{1} .}
\end{aligned}
$$

However, due to Jacobi identity we get $a=b=c=0$. Thus, there are no non-trivial deformations in Type III case, and dimension 7 of symmetry algebra is not realized.

Similarly, for types I and II we have the one-dimensional annihilator $\mathfrak{a}_{0}$ spanned by $T=Z_{1}-Z_{3}$. Using the same argument, we get a 4-parameter family of non-trivial deformations $\mathfrak{s}$ given by:

$$
\begin{aligned}
& {\left[T, E_{1}\right]=-E_{1}, \quad\left[T, E_{2}\right]=-E_{2}, \quad\left[T, F_{1}\right]=F_{1}, \quad\left[T, F_{2}\right]=F_{2},} \\
& {\left[E_{1}, F_{1}\right]=U+a_{11} T, \quad\left[E_{1}, F_{2}\right]=a_{12} T, \quad\left[E_{2}, F_{1}\right]=a_{21} T, \quad\left[E_{2}, F_{2}\right]=U+a_{22} T,} \\
& {\left[E_{1}, U\right]=-a_{22} E_{1}+a_{12} E_{2}, \quad\left[E_{2}, U\right]=a_{21} E_{1}-a_{11} E_{2},} \\
& {\left[F_{1}, U\right]=a_{22} F_{1}-a_{21} F_{2}, \quad\left[F_{2}, U\right]=-a_{12} F_{1}+a_{11} F_{2} .}
\end{aligned}
$$

Replacing $U$ by $U+\lambda T$, we may assume that $a_{22}=-a_{11}$. Each of these deformations $\mathfrak{s}$ defines an $S$-invariant ILC structure on the homogeneous space $S / S_{0}$, where $S$ is the corresponding Lie group and $S_{0}$ is the subgroup corresponding to the 1-dimensional subalgebra spanned by $T$. The linear map $\alpha: \mathfrak{s} \rightarrow \mathfrak{s l}_{4}$ given by

$$
\begin{aligned}
& E_{1} \mapsto E_{1}{ }^{0}-\frac{1}{2} a_{11} E_{1}{ }^{3}-\frac{1}{2} a_{12} E_{2}{ }^{3}, \quad E_{2} \mapsto E_{2}{ }^{0}-\frac{1}{2} a_{21} E_{1}{ }^{3}+\frac{1}{2} a_{11} E_{2}{ }^{3}, \\
& F_{1} \mapsto E_{0}{ }^{1}-\frac{1}{2} a_{11} E_{0}{ }^{1}-\frac{1}{2} a_{21} E_{0}{ }^{2}, \quad F_{2} \mapsto E_{0}{ }^{2}-\frac{1}{2} a_{12} E_{0}{ }^{1}+\frac{1}{2} a_{11} E_{0}{ }^{2}, \\
& U \mapsto-E_{3}{ }^{0}-\frac{1}{2}\left(a_{11} E_{1}{ }^{1}+a_{21} E_{1}{ }^{2}+a_{12} E_{2}{ }^{1}-a_{11} E_{2}{ }^{2}\right)-\frac{1}{4}\left(a_{11}^{2}+a_{12} a_{21}\right) E_{0}{ }^{3}
\end{aligned}
$$

is in fact a Lie algebra homomorphism. Hence, all these deformations are in fact trivial and yield the flat ILC structure [4, Sec.1.5.15-16]. This contradicts the type I or II assumption.

We exclude types II and I from further consideration, since no multiply transitive models exist. 
3.2. Curvature module. Since ILC structures are torsion-free geometries, a result of Čap [3, Sec. 3.2 corollary] implies that the curvature function $\kappa$ takes values in the $P$-module $\mathbb{K} \subset \Lambda^{2} \mathfrak{p}_{+} \otimes \mathfrak{p}$ generated by $\mathbb{W}$. We refer to $\mathbb{K}$ as the curvature module.

From Kostant's theorem, $\mathbb{W}$ has lowest weight vector $\phi_{0}=E_{0}{ }^{1} \wedge E_{2}{ }^{3} \otimes E_{2}{ }^{1} \leftrightarrow \mathrm{s}^{4}$, and we generate all of $\mathbb{W}$ by applying the raising operator $E_{1}{ }^{2} \leftrightarrow \mathrm{r}_{\mathrm{s}}$. The result of applying the raising operators $E_{0}{ }^{1}, E_{0}{ }^{2}, E_{1}{ }^{3}, E_{2}{ }^{3}, E_{0}{ }^{3} \in \mathfrak{p}_{+}$to $\mathbb{W}$ is given in Table 3.1. Introduce coordinates on $\mathbb{K}$ (26-dimensional):

\begin{tabular}{|c|l|}
\hline Quartic & $A=A_{1} \mathrm{r}^{4}+4 A_{2} \mathrm{r}^{3} \mathrm{~s}+6 A_{3} \mathrm{r}^{2} \mathrm{~s}^{2}+4 A_{4} \mathrm{rs}^{3}+A_{5} \mathrm{~s}^{4}$ \\
\hline Cubic & $B=4\left(B_{1} \mathrm{r}^{3}+3 B_{2} \mathrm{r}^{2} \mathrm{~s}+3 B_{3} \mathrm{rs}^{2}+B_{4} \mathrm{~s}^{3}\right) \mathrm{w}_{1}$ \\
& $B^{\prime}=4\left(B_{5} \mathrm{r}^{3}+3 B_{6} \mathrm{r}^{2} \mathrm{~s}+3 B_{7} \mathrm{rs}^{2}+B_{8} \mathrm{~s}^{3}\right) \mathrm{w}_{2}$ \\
\hline Quadratic & $C=6\left(C_{1} \mathrm{r}^{2}+2 C_{2} \mathrm{rs}+C_{3} \mathrm{~s}^{2}\right) \mathrm{w}_{1}^{2}$ \\
& $\widehat{C}=12\left(C_{4} \mathrm{r}^{2}+2 C_{5} \mathrm{rs}+C_{6} \mathrm{~s}^{2}\right) \mathrm{w}_{1} \mathrm{w}_{2}$ \\
& $C^{\prime}=6\left(C_{7} \mathrm{r}^{2}+2 C_{8} \mathrm{rs}+C_{9} \mathrm{~s}^{2}\right) \mathrm{w}_{2}^{2}$ \\
\hline Linear & $D=12\left(D_{1} \mathrm{r}+D_{2} \mathrm{~s}\right) \mathrm{w}_{1}^{2} \mathrm{w}_{2}$ \\
& $D^{\prime}=12\left(D_{3} \mathrm{r}+D_{4} \mathrm{~s}\right) \mathrm{w}_{1} \mathrm{w}_{2}^{2}$ \\
\hline
\end{tabular}

\begin{tabular}{|c|c|c|}
\hline$\left(Z_{1}, Z_{3}\right)$-grade & Label & 2-chain \\
\hline$\overline{(7+1,+1)}$ & $\begin{array}{c}r^{4} \\
4 r^{3} s \\
6 r^{2} s^{2} \\
4 r s^{3} \\
s^{4}\end{array}$ & $\begin{array}{l}E_{0}^{2} \wedge E_{1}^{3} \otimes E_{1}^{2} \\
-\Omega \otimes E_{1}^{2}-E_{0}^{2} \wedge E_{1}^{3} \otimes \mathrm{H} \\
-E_{0}{ }^{1} \wedge E_{2}^{3} \otimes E_{1}^{2}-E_{0}^{2} \wedge E_{1}^{3} \otimes E_{2}^{1}+\Omega \otimes \mathrm{H} \\
\Omega \otimes E_{2}{ }^{1}+E_{0}{ }^{1} \wedge E_{2}^{3} \otimes \mathrm{H} \\
E_{0}{ }^{1} \wedge E_{2}^{3} \otimes E_{2}{ }^{1}\end{array}$ \\
\hline$(+2,+1)$ & $\begin{array}{c}4 r^{3} w_{1} \\
12 r^{2} s w_{1} \\
12 r s^{2} w_{1} \\
4 s^{3} w_{1}\end{array}$ & $\begin{array}{l}E_{0}{ }^{2} \wedge E_{0}{ }^{3} \otimes E_{1}{ }^{2}+E_{0}{ }^{2} \wedge E_{1}^{3} \otimes E_{0}^{2} \\
-E_{0}{ }^{1} \wedge E_{0}^{3} \otimes E_{1}^{2}-E_{0}{ }^{2} \wedge E_{1}^{3} \otimes E_{0}{ }^{1}-\Omega \otimes E_{0}{ }^{2}-E_{0}{ }^{2} \wedge E_{0}{ }^{3} \otimes \mathrm{H} \\
-E_{0}{ }^{2} \wedge E_{0}{ }^{3} \otimes E_{2}{ }^{1}-E_{0}{ }^{1} \wedge E_{2}^{3} \otimes E_{0}{ }^{2}+\Omega \otimes E_{0}{ }^{1}+E_{0}{ }^{1} \wedge E_{0}{ }^{3} \otimes \mathrm{H} \\
E_{0}{ }^{1} \wedge E_{0}{ }^{3} \otimes E_{2}{ }^{1}+E_{0}{ }^{1} \wedge E_{2}{ }^{3} \otimes E_{0}{ }^{1}\end{array}$ \\
\hline$(+1,+2)$ & $\begin{array}{c}4 r^{3} w_{2} \\
12 r^{2} s w_{2} \\
12 r s^{2} w_{2} \\
4 s^{3} w_{2}\end{array}$ & $\begin{array}{l}E_{1}{ }^{3} \wedge E_{0}{ }^{3} \otimes E_{1}{ }^{2}+E_{1}{ }^{3} \wedge E_{0}{ }^{2} \otimes E_{1}{ }^{3} \\
E_{2}{ }^{3} \wedge E_{0}{ }^{3} \otimes E_{1}{ }^{2}+E_{1}{ }^{3} \wedge E_{0}{ }^{2} \otimes E_{2}{ }^{3}+\Omega \otimes E_{1}{ }^{3}-E_{1}{ }^{3} \wedge E_{0}{ }^{3} \otimes \mathrm{H} \\
-E_{1}{ }^{3} \wedge E_{0}{ }^{3} \otimes E_{2}{ }^{1}-E_{2}{ }^{3} \wedge E_{0}{ }^{1} \otimes E_{1}{ }^{3}+\Omega \otimes E_{2}{ }^{3}-E_{2}{ }^{3} \wedge E_{0}{ }^{3} \otimes \mathrm{H} \\
-E_{2}{ }^{3} \wedge E_{0}{ }^{3} \otimes E_{2}{ }^{1}-E_{2}{ }^{3} \wedge E_{0}{ }^{1} \otimes E_{2}{ }^{3}\end{array}$ \\
\hline$(+3,+1)$ & $\begin{array}{l}6 \mathrm{r}^{2} \mathrm{w}_{1}^{2} \\
12 r s w_{1}^{2} \\
6 \mathrm{~s}^{2} \mathrm{w}_{1}^{2}\end{array}$ & $\begin{array}{l}E_{0}^{2} \wedge E_{0}^{3} \otimes E_{0}^{2} \\
E_{0}{ }^{3} \wedge E_{0}^{2} \otimes E_{0}^{1}+E_{0}^{3} \wedge E_{0}^{1} \otimes E_{0}^{2} \\
E_{0}^{1} \wedge E_{0}^{3} \otimes E_{0}^{1}\end{array}$ \\
\hline$(+2,+2)$ & $\begin{array}{l}12 r^{2} w_{1} w_{2} \\
24 r s w_{1} w_{2} \\
12 s^{2} w_{1} w_{2}\end{array}$ & $\begin{array}{l}E_{1}^{3} \wedge E_{0}{ }^{3} \otimes E_{0}{ }^{2}+E_{0}{ }^{3} \wedge E_{0}{ }^{2} \otimes E_{1}{ }^{3}+E_{1}{ }^{3} \wedge E_{0}{ }^{2} \otimes E_{0}{ }^{3} \\
E_{0}{ }^{3} \wedge E_{0}{ }^{2} \otimes E_{2}{ }^{3}-E_{0}{ }^{3} \wedge E_{2}{ }^{3} \otimes E_{0}{ }^{2}+\Omega \otimes E_{0}{ }^{3} \\
\quad+E_{0}{ }^{3} \wedge E_{1}{ }^{3} \otimes E_{0}{ }^{1}-E_{0}{ }^{3} \wedge E_{0}{ }^{1} \otimes E_{1}{ }^{3} \\
E_{0}{ }^{1} \wedge E_{0}{ }^{3} \otimes E_{2}{ }^{3}+E_{0}{ }^{3} \wedge E_{2}{ }^{3} \otimes E_{0}{ }^{1}+E_{0}{ }^{1} \wedge E_{2}{ }^{3} \otimes E_{0}{ }^{3}\end{array}$ \\
\hline$(+1,+3)$ & $\begin{array}{l}6 \mathrm{r}^{2} \mathrm{w}_{2}^{2} \\
12 r s w_{2}^{2} \\
6 \mathrm{~s}^{2} \mathrm{w}_{2}^{2}\end{array}$ & $\begin{array}{l}E_{0}{ }^{3} \wedge E_{1}{ }^{3} \otimes E_{1}{ }^{3} \\
E_{0}{ }^{3} \wedge E_{1}{ }^{3} \otimes E_{2}{ }^{3}+E_{0}{ }^{3} \wedge E_{2}{ }^{3} \otimes E_{1}{ }^{3} \\
E_{0}{ }^{3} \wedge E_{2}{ }^{3} \otimes E_{2}{ }^{3}\end{array}$ \\
\hline$(+3,+2)$ & $\begin{array}{l}12 \mathrm{rw}_{1}^{2} \mathrm{w}_{2} \\
12 \mathrm{sw}_{1}^{2} \mathrm{w}_{2}\end{array}$ & $\begin{array}{l}E_{0}{ }^{3} \wedge E_{0}{ }^{2} \otimes E_{0}{ }^{3} \\
E_{0}{ }^{1} \wedge E_{0}{ }^{3} \otimes E_{0}{ }^{3}\end{array}$ \\
\hline$\overline{(+2,+3)}$ & $\begin{array}{l}12 \mathrm{rw}_{1} \mathrm{w}_{2}^{2} \\
12 \mathrm{sw}_{1} \mathrm{w}_{2}^{2} \\
\end{array}$ & $\begin{array}{l}E_{0}{ }^{3} \wedge E_{1}{ }^{3} \otimes E_{0}{ }^{3} \\
E_{0}{ }^{3} \wedge E_{2}{ }^{3} \otimes E_{0}{ }^{3}\end{array}$ \\
\hline $\begin{array}{r}\text { Notati } \\
\mathfrak{p} \text {-module descr } \\
\mathfrak{s l}_{2} \text {-acti } \\
\mathfrak{p}_{+} \text {-acti }\end{array}$ & $\begin{array}{l}\text { n: } \\
\text { ption: } \\
n:\end{array}$ & $\begin{array}{l}\Omega:=E_{0}{ }^{1} \wedge E_{1}{ }^{3}-E_{0}{ }^{2} \wedge E_{2}{ }^{3} \\
\text { Degree } 4 \text { polynomials in } \mathrm{r}, \mathrm{s}, \mathrm{w}_{1}, \mathrm{w}_{2} \text { modulo } \mathrm{w}_{1}^{3}, \mathrm{w}_{2}^{3}, \mathrm{w}_{1}^{3} \mathrm{w}_{2}, \mathrm{w}_{1}^{2} \mathrm{w}_{2}^{2}, \mathrm{w}_{1} \mathrm{w}_{2}^{3} \\
\mathrm{H}=E_{1}{ }^{1}-E_{2}^{2} \leftrightarrow \mathrm{r}_{\mathrm{r}}-\mathrm{s} \partial_{\mathrm{s}}, \quad \mathrm{X}=E_{1}{ }^{2} \leftrightarrow \mathrm{r}_{\mathrm{s}}, \quad \mathrm{Y}=E_{2}{ }^{1} \leftrightarrow \mathrm{s}_{\mathrm{r}} \\
E_{0}{ }^{1} \leftrightarrow \mathrm{w}_{1} \partial_{\mathrm{r}}, \quad E_{0}{ }^{2} \leftrightarrow \mathrm{w}_{1} \partial_{\mathrm{s}}, \quad E_{1}{ }^{3} \leftrightarrow-\mathrm{w}_{2} \partial_{\mathrm{s}}, \quad E_{2}{ }^{3} \leftrightarrow \mathrm{w}_{2} \partial_{\mathrm{r}},\end{array}$ \\
\hline
\end{tabular}

TABLE 3.1. The curvature module for ILC structures 
3.3. Structure equations. Write the Cartan connection $\omega \in \Omega^{1}(\mathcal{G} ; \mathfrak{g})$ as

$$
\omega=\left[\omega_{b}^{a}\right]=\left[\begin{array}{cccc}
\frac{3 \zeta_{1}+\zeta_{2}}{4} & \tau_{1} & \tau_{2} & \tau_{5} \\
\varpi_{1} & \nu_{1}+\frac{\zeta_{2}-\zeta_{1}}{4} & \nu_{2} & \tau_{3} \\
\varpi_{2} & \nu_{3} & -\nu_{1}+\frac{\zeta_{2}-\zeta_{1}}{4} & \tau_{4} \\
\varpi_{5} & \varpi_{3} & \varpi_{4} & -\frac{\zeta_{1}+3 \zeta_{2}}{4}
\end{array}\right] .
$$

Decompose $K=K_{b}^{a} E_{a}{ }^{b}$, where $K_{b}^{a} \in \Omega^{2}(\mathcal{G})$. By torsion-freeness, $K^{1}{ }_{0}=K_{0}^{2}=K^{3}{ }_{0}=K^{3}{ }_{1}=$ $K^{3}{ }_{2}=0$, and for ILC structures $K_{0}^{0}=K^{3}{ }_{3}=0$. The structure equations are $d \omega^{a}{ }_{b}=-\omega^{a}{ }_{c} \wedge \omega^{c}{ }_{b}+$ $K^{a}{ }_{b}$, i.e.

$$
\begin{array}{ll}
d \tau_{1}=\left(\nu_{1}-\zeta_{1}\right) \wedge \tau_{1}+\nu_{3} \wedge \tau_{2}-\tau_{5} \wedge \varpi_{3}+K^{0}{ }_{1} & d \varpi_{1}=\left(\zeta_{1}-\nu_{1}\right) \wedge \varpi_{1}-\nu_{2} \wedge \varpi_{2}-\tau_{3} \wedge \varpi_{5} \\
d \tau_{2}=\nu_{2} \wedge \tau_{1}-\left(\zeta_{1}+\nu_{1}\right) \wedge \tau_{2}-\tau_{5} \wedge \varpi_{4}+K^{0}{ }_{2} & d \varpi_{2}=-\nu_{3} \wedge \varpi_{1}+\left(\nu_{1}+\zeta_{1}\right) \wedge \varpi_{2}-\tau_{4} \wedge \varpi_{5} \\
d \tau_{3}=\tau_{5} \wedge \varpi_{1}-\left(\nu_{1}+\zeta_{2}\right) \wedge \tau_{3}-\nu_{2} \wedge \tau_{4}+K^{1}{ }_{3} & d \varpi_{3}=\left(\nu_{1}+\zeta_{2}\right) \wedge \varpi_{3}+\nu_{3} \wedge \varpi_{4}+\tau_{1} \wedge \varpi_{5} \\
d \tau_{4}=\tau_{5} \wedge \varpi_{2}-\nu_{3} \wedge \tau_{3}+\left(\nu_{1}-\zeta_{2}\right) \wedge \tau_{4}+K^{2}{ }_{3} & d \varpi_{4}=\nu_{2} \wedge \varpi_{3}+\left(\zeta_{2}-\nu_{1}\right) \wedge \varpi_{4}+\tau_{2} \wedge \varpi_{5} \\
d \tau_{5}=-\tau_{1} \wedge \tau_{3}-\tau_{2} \wedge \tau_{4}-\left(\zeta_{1}+\zeta_{2}\right) \wedge \tau_{5}+K^{0}{ }_{3} & d \varpi_{5}=\varpi_{1} \wedge \varpi_{3}+\varpi_{2} \wedge \varpi_{4}+\left(\zeta_{1}+\zeta_{2}\right) \wedge \varpi_{5} \\
d \zeta_{1}=-\frac{3}{2} \tau_{1} \wedge \varpi_{1}-\frac{3}{2} \tau_{2} \wedge \varpi_{2}+\frac{1}{2} \tau_{3} \wedge \varpi_{3}+\frac{1}{2} \tau_{4} \wedge \varpi_{4}-\tau_{5} \wedge \varpi_{5} \\
d \zeta_{2}=\frac{1}{2} \tau_{1} \wedge \varpi_{1}+\frac{1}{2} \tau_{2} \wedge \varpi_{2}-\frac{3}{2} \tau_{3} \wedge \varpi_{3}-\frac{3}{2} \tau_{4} \wedge \varpi_{4}-\tau_{5} \wedge \varpi_{5} \\
d \nu_{1}=\frac{1}{2} \tau_{1} \wedge \varpi_{1}-\frac{1}{2} \tau_{2} \wedge \varpi_{2}-\frac{1}{2} \tau_{3} \wedge \varpi_{3}+\frac{1}{2} \tau_{4} \wedge \varpi_{4}-\nu_{2} \wedge \nu_{3}+\frac{1}{2} K^{1}{ }_{1}-\frac{1}{2} K^{2}{ }_{2} \\
d \nu_{2}=\tau_{2} \wedge \varpi_{1}-2 \nu_{1} \wedge \nu_{2}-\tau_{3} \wedge \varpi_{4}+K^{1}{ }_{2} & \\
d \nu_{3}=\tau_{1} \wedge \varpi_{2}+2 \nu_{1} \wedge \nu_{3}-\tau_{4} \wedge \varpi_{3}+K^{2}{ }_{1} &
\end{array}
$$

To convert from $\kappa: \mathcal{G} \rightarrow \bigwedge^{2} \mathfrak{p}_{+} \otimes \mathfrak{g}$ to $K \in \Omega^{2}(\mathcal{G} ; \mathfrak{g})$, the Killing form on $\mathfrak{s l}_{4}$ induces $(\mathfrak{g} / \mathfrak{p})^{*} \cong \mathfrak{p}_{+}:$ $E_{0}{ }^{1} \leftrightarrow \omega^{1}{ }_{0}=\varpi_{1}, \quad E_{0}{ }^{2} \leftrightarrow \omega^{2}{ }_{0}=\varpi_{2}, \quad E_{1}{ }^{3} \leftrightarrow \omega^{3}{ }_{1}=\varpi_{3}, \quad E_{2}{ }^{3} \leftrightarrow \omega^{3}{ }_{2}=\varpi_{4}, \quad E_{0}{ }^{3} \leftrightarrow \omega^{3}{ }_{0}=\varpi_{5}$.

Writing $\varpi_{k l}:=\varpi_{k} \wedge \varpi_{l}$ for $1 \leq k, l \leq 5$, we have

$$
\begin{array}{r}
K_{1}^{1}=-K^{2}{ }_{2}=-A_{2} \varpi_{23}+A_{3}\left(\varpi_{13}-\varpi_{24}\right)+A_{4} \varpi_{14}-B_{2} \varpi_{25}+B_{3} \varpi_{15}-B_{6} \varpi_{35}-B_{7} \varpi_{45} \\
K^{1}{ }_{2}=+A_{1} \varpi_{23}-A_{2}\left(\varpi_{13}-\varpi_{24}\right)-A_{3} \varpi_{14}+B_{1} \varpi_{25}-B_{2} \varpi_{15}+B_{5} \varpi_{35}+B_{6} \varpi_{45} \\
K^{2}{ }_{1}=-A_{3} \varpi_{23}+A_{4}\left(\varpi_{13}-\varpi_{24}\right)+A_{5} \varpi_{14}-B_{3} \varpi_{25}+B_{4} \varpi_{15}-B_{7} \varpi_{35}-B_{8} \varpi_{45} \\
K^{0}{ }_{1}=-B_{2} \varpi_{23}+B_{3}\left(\varpi_{13}-\varpi_{24}\right)+B_{4} \varpi_{14}-C_{2} \varpi_{25}+C_{3} \varpi_{15}-C_{5} \varpi_{35}-C_{6} \varpi_{45} \\
K^{0}{ }_{2}=+B_{1} \varpi_{23}-B_{2}\left(\varpi_{13}-\varpi_{24}\right)-B_{3} \varpi_{14}+C_{1} \varpi_{25}-C_{2} \varpi_{15}+C_{4} \varpi_{35}+C_{5} \varpi_{45} \\
K^{1}{ }_{3}=-B_{5} \varpi_{23}+B_{6}\left(\varpi_{13}-\varpi_{24}\right)+B_{7} \varpi_{14}-C_{4} \varpi_{25}+C_{5} \varpi_{15}-C_{7} \varpi_{35}-C_{8} \varpi_{45} \\
K^{2}{ }_{3}=-B_{6} \varpi_{23}+B_{7}\left(\varpi_{13}-\varpi_{24}\right)+B_{8} \varpi_{14}-C_{5} \varpi_{25}+C_{6} \varpi_{15}-C_{8} \varpi_{35}-C_{9} \varpi_{45} \\
K^{0}{ }_{3}=-C_{4} \varpi_{23}+C_{5}\left(\varpi_{13}-\varpi_{24}\right)+C_{6} \varpi_{14}-D_{1} \varpi_{25}+D_{2} \varpi_{15}-D_{3} \varpi_{35}-D_{4} \varpi_{45}
\end{array}
$$

Recall from Section 2.3 that $\kappa$ is $P$-equivariant. Let $\lambda$ be the $P$-representation $\mathbb{K}$. Then

$$
R_{p}^{*} \kappa=\left.\lambda\left(p^{-1}\right) \cdot \kappa \Rightarrow \frac{d}{d \epsilon}\right|_{\epsilon=0} R_{\exp (\epsilon X)}^{*} \kappa=-\lambda^{\prime}(X) \cdot \kappa .
$$

We let $\delta$ refer to the infinitesimal $\mathfrak{p}$-action. Given $X \in \mathfrak{p}$ as in (3.1), we obtain Table 3.2.

On $\mathcal{G}$, the curvature coefficients $A, B, C, D$ will satisfy structure equations that also account for variation in the horizontal direction. These are immediately deduced from Table 3.2. For example,

$$
d\left(A_{1}\right)=\delta\left(A_{1}\right)+\alpha_{1}=\left(\zeta_{1}+\zeta_{2}+4 \nu_{1}\right) A_{1}+4 \nu_{2} A_{2}+\alpha_{1} .
$$

Here, $\alpha_{1}$ is a semi-basic form, i.e. it is a linear combination (with coefficients that are functions on $\mathcal{G})$ of the $\varpi_{i}$. We have abused notation in (3.5) by taking a slightly different meaning for $\delta\left(A_{1}\right)$ : we have taken the corresponding formula in Table 3.2 and replaced Lie algebra parameters by their corresponding forms in the Cartan connection. This abuse is justified by axiom (CC.3) in the Cartan connection definition. Similarly, we will write

$$
d\left(A_{i}\right)=\delta\left(A_{i}\right)+\alpha_{i}, \quad d\left(B_{i}\right)=\delta\left(B_{i}\right)+\beta_{i}, \quad d\left(C_{i}\right)=\delta\left(C_{i}\right)+\gamma_{i}, \quad d\left(D_{i}\right)=\delta\left(D_{i}\right)+\delta_{i} .
$$

(The repetition of $\delta$ in the last formula is slightly unfortunate, but should not cause much confusion.) 


$$
\begin{aligned}
& -\delta A_{1}=\left(z_{1}+z_{2}+4 v_{1}\right) A_{1}+4 v_{2} A_{2} \\
& -\delta A_{2}=v_{3} A_{1}+\left(z_{1}+z_{2}+2 v_{1}\right) A_{2}+3 v_{2} A_{3} \\
& -\delta A_{3}=2 v_{3} A_{2}+\left(z_{1}+z_{2}\right) A_{3}+2 v_{2} A_{4} \\
& -\delta A_{4}=3 v_{3} A_{3}+\left(z_{1}+z_{2}-2 v_{1}\right) A_{4}+v_{2} A_{5} \\
& -\delta A_{5}=4 v_{3} A_{4}+\left(z_{1}+z_{2}-4 v_{1}\right) A_{5} \\
& -\delta B_{1}=\left(2 z_{1}+z_{2}+3 v_{1}\right) B_{1}+3 v_{2} B_{2}+t_{1} A_{1}+t_{2} A_{2} \\
& -\delta B_{2}=v_{3} B_{1}+\left(2 z_{1}+z_{2}+v_{1}\right) B_{2}+2 v_{2} B_{3}+t_{1} A_{2}+t_{2} A_{3} \\
& -\delta B_{3}=2 v_{3} B_{2}+\left(2 z_{1}+z_{2}-v_{1}\right) B_{3}+v_{2} B_{4}+t_{1} A_{3}+t_{2} A_{4} \\
& -\delta B_{4}=3 v_{3} B_{3}+\left(2 z_{1}+z_{2}-3 v_{1}\right) B_{4}+t_{1} A_{4}+t_{2} A_{5} \\
& -\delta B_{5}=\left(z_{1}+2 z_{2}+3 v_{1}\right) B_{5}+3 v_{2} B_{6}+t_{4} A_{1}-t_{3} A_{2} \\
& -\delta B_{6}=v_{3} B_{5}+\left(z_{1}+2 z_{2}+v_{1}\right) B_{6}+2 v_{2} B_{7}+t_{4} A_{2}-t_{3} A_{3} \\
& -\delta B_{7}=2 v_{3} B_{6}+\left(z_{1}+2 z_{2}-v_{1}\right) B_{7}+v_{2} B_{8}+t_{4} A_{3}-t_{3} A_{4} \\
& -\delta B_{8}=3 v_{3} B_{7}+\left(z_{1}+2 z_{2}-3 v_{1}\right) B_{8}+t_{4} A_{4}-t_{3} A_{5} \\
& -\delta C_{1}=\left(3 z_{1}+z_{2}+2 v_{1}\right) C_{1}+2 v_{2} C_{2}+2 t_{1} B_{1}+2 t_{2} B_{2} \\
& -\delta C_{2}=v_{3} C_{1}+\left(3 z_{1}+z_{2}\right) C_{2}+v_{2} C_{3}+2 t_{1} B_{2}+2 t_{2} B_{3} \\
& -\delta C_{3}=2 v_{3} C_{2}+\left(3 z_{1}+z_{2}-2 v_{1}\right) C_{3}+2 t_{1} B_{3}+2 t_{2} B_{4} \\
& -\delta C_{4}=\left(2 z_{1}+2 z_{2}+2 v_{1}\right) C_{4}+2 v_{2} C_{5}+t_{4} B_{1}-t_{3} B_{2}+t_{1} B_{5}+t_{2} B_{6} \\
& -\delta C_{5}=v_{3} C_{4}+\left(2 z_{1}+2 z_{2}\right) C_{5}+v_{2} C_{6}+t_{4} B_{2}-t_{3} B_{3}+t_{1} B_{6}+t_{2} B_{7} \\
& -\delta C_{6}=2 v_{3} C_{5}+\left(2 z_{1}+2 z_{2}-2 v_{1}\right) C_{6}+t_{4} B_{3}-t_{3} B_{4}+t_{1} B_{7}+t_{2} B_{8} \\
& -\delta C_{7}=\left(z_{1}+3 z_{2}+2 v_{1}\right) C_{7}+2 v_{2} C_{8}+2 t_{4} B_{5}-2 t_{3} B_{6} \\
& -\delta C_{8}=v_{3} C_{7}+\left(z_{1}+3 z_{2}\right) C_{8}+v_{2} C_{9}+2 t_{4} B_{6}-2 t_{3} B_{7} \\
& -\delta C_{9}=2 v_{3} C_{8}+\left(z_{1}+3 z_{2}-2 v_{1}\right) C_{9}+2 t_{4} B_{7}-2 t_{3} B_{8} \\
& -\delta D_{1}=\left(3 z_{1}+2 z_{2}+v_{1}\right) D_{1}+v_{2} D_{2}+t_{4} C_{1}-t_{3} C_{2}+2 t_{1} C_{4}+2 t_{2} C_{5} \\
& -\delta D_{2}=v_{3} D_{1}+\left(3 z_{1}+2 z_{2}-v_{1}\right) D_{2}+t_{4} C_{2}-t_{3} C_{3}+2 t_{1} C_{5}+2 t_{2} C_{6} \\
& -\delta D_{3}=\left(2 z_{1}+3 z_{2}+v_{1}\right) D_{3}+v_{2} D_{4}+2 t_{4} C_{4}-2 t_{3} C_{5}+t_{1} C_{7}+t_{2} C_{8} \\
& -\delta D_{4}=v_{3} D_{3}+\left(2 z_{1}+3 z_{2}-v_{1}\right) D_{4}+2 t_{4} C_{5}-2 t_{3} C_{6}+t_{1} C_{8}+t_{2} C_{9}
\end{aligned}
$$

TABLE 3.2. Vertical derivatives of curvature coefficients

3.4. Duality. The pullback of the subbundles $E, V \subset T M$ via the projection $\pi: \mathcal{G} \rightarrow M$ are

$$
\pi^{-1}(E)=\left\{\varpi_{3}=\varpi_{4}=\varpi_{5}=0\right\}, \quad \pi^{-1}(V)=\left\{\varpi_{1}=\varpi_{2}=\varpi_{5}=0\right\} .
$$

These are interchanged by the duality transformation, a representative of which is

$$
\begin{aligned}
& \iota:\left(\varpi_{1}, \varpi_{2}, \varpi_{3}, \varpi_{4}, \varpi_{5}, \zeta_{1}, \zeta_{2}, \nu_{1}, \nu_{2}, \nu_{3}, \tau_{1}, \tau_{2}, \tau_{3}, \tau_{4}, \tau_{5}\right) \\
& \quad \mapsto\left(\varpi_{3}, \varpi_{4}, \varpi_{1}, \varpi_{2},-\varpi_{5}, \zeta_{2}, \zeta_{1},-\nu_{1},-\nu_{3},-\nu_{2}, \tau_{3}, \tau_{4}, \tau_{1}, \tau_{2},-\tau_{5}\right),
\end{aligned}
$$

which induces

$$
\begin{aligned}
\left(A_{1}, A_{2}, A_{3}, A_{4}, A_{5}\right) & \mapsto\left(A_{5},-A_{4}, A_{3},-A_{2}, A_{1}\right) \\
\left(B_{1}, B_{2}, B_{3}, B_{4}, B_{5}, B_{6}, B_{7}, B_{8}\right) & \mapsto\left(-B_{8}, B_{7},-B_{6}, B_{5}, B_{4},-B_{3}, B_{2},-B_{1}\right) \\
\left(C_{1}, C_{2}, C_{3}, C_{4}, C_{5}, C_{6}, C_{7}, C_{8}, C_{9}\right) & \mapsto\left(C_{9},-C_{8}, C_{7},-C_{6}, C_{5},-C_{4}, C_{3},-C_{2}, C_{1}\right) \\
\left(D_{1}, D_{2}, D_{3}, D_{4}\right) & \mapsto\left(D_{4},-D_{3},-D_{2}, D_{1}\right)
\end{aligned}
$$

In particular, the induced action on the quartic is realizable by a $G_{0}$-transformation, namely that induced by $\rho:(x, y) \mapsto(y,-x)$. Since any $G_{0}$-transformation preserves root type, this proves:

Proposition 3.2. The duality transformation preserves root type.

However, the duality transformation differs from $(x, y) \mapsto(y,-x)$ on $B, C, D$ coefficients:

$$
\begin{aligned}
\left(B_{1}, B_{2}, B_{3}, B_{4}, B_{5}, B_{6}, B_{7}, B_{8}\right) & \mapsto\left(-B_{4}, B_{3},-B_{2}, B_{1},-B_{8}, B_{7},-B_{6}, B_{5}\right) \\
\left(C_{1}, C_{2}, C_{3}, C_{4}, C_{5}, C_{6}, C_{7}, C_{8}, C_{9}\right) & \mapsto\left(C_{3},-C_{2}, C_{1}, C_{6},-C_{5}, C_{4}, C_{9},-C_{8}, C_{7}\right) \\
\left(D_{1}, D_{2}, D_{3}, D_{4}\right) & \mapsto\left(-D_{2}, D_{1},-D_{4}, D_{3}\right)
\end{aligned}
$$


Note that the composition $\rho \circ \iota$ preserves $A$ and induces

$$
\begin{aligned}
\left(B_{1}, B_{2}, B_{3}, B_{4}, B_{5}, B_{6}, B_{7}, B_{8}\right) & \mapsto\left(-B_{5},-B_{6},-B_{7},-B_{8}, B_{1}, B_{2}, B_{3}, B_{4}\right) \\
\left(C_{1}, C_{2}, C_{3}, C_{4}, C_{5}, C_{6}, C_{7}, C_{8}, C_{9}\right) & \mapsto\left(C_{7}, C_{8}, C_{9},-C_{4},-C_{5},-C_{6}, C_{1}, C_{2}, C_{3}\right) \\
\left(D_{1}, D_{2}, D_{3}, D_{4}\right) & \mapsto\left(D_{3}, D_{4},-D_{1},-D_{2}\right)
\end{aligned}
$$

\section{Cartan analysis}

Starting with the (regular, normal) Cartan geometry $(\mathcal{G} \rightarrow M, \omega)$ which is an equivalent description of any ILC structure, the goal is to classify all homogeneous sub-bundles of total dimension at least six that are obtained via natural reductions of the structure group $P$. We give an outline of how this is achieved in the type $\mathrm{N}$ case. The analysis for types D and III are similar, so we only provide a few details on how the analysis is begun in these cases. Types II and I do not contain any multiply transitive structures. The reader interested in the full details of the Cartan analysis is encouraged to examine the Maple files which accompany the arXiv submission of this paper.

4.1. Type $\mathbf{N}$ reduction. Using the $P$-action $\left(G_{0}\right.$-action), we can always normalize $A=y^{4}$, i.e. $A_{5}=1, A_{4}=A_{3}=A_{2}=A_{1}=0.3$ Now $0=d\left(A_{i}\right)$ are equivalent to:

$$
\alpha_{1}=\alpha_{2}=\alpha_{3}=0, \quad \nu_{2}=\alpha_{4}, \quad \nu_{1}=\frac{1}{4}\left(\zeta_{1}+\zeta_{2}-\alpha_{5}\right) .
$$

Differentiating the $\nu_{1}$-relation in (4.1) yields the vertical action on coefficients in $\alpha_{5}=a_{5 j} \varpi_{j}$. (More precisely, we calculate $0=d\left(\nu_{1}-\frac{1}{4}\left(\zeta_{1}+\zeta_{2}-\alpha_{5}\right)\right) \wedge \varpi_{i} \wedge \varpi_{j} \wedge \varpi_{k} \wedge \varpi_{\ell}$, where $1 \leq i, j, k, l \leq 5$.)

$$
\begin{aligned}
& \delta a_{51}=\left(\frac{z_{2}-3 z_{1}}{4}\right) a_{51}+\left(a_{52}-4 a_{41}\right) v_{3}-3 t_{1}, \quad \delta a_{52}=-\left(\frac{5 z_{1}+z_{2}}{4}\right) a_{52}-4 a_{42} v_{3}+t_{2}, \\
& \delta a_{54}=\left(\frac{z_{1}-3 z_{2}}{4}\right) a_{54}-\left(a_{53}+4 a_{44}\right) v_{3}-3 t_{4}, \quad \delta a_{53}=-\left(\frac{z_{1}+5 z_{2}}{4}\right) a_{53}-4 a_{43} v_{3}+t_{3}, \\
& \delta a_{55}=-a_{55}\left(z_{1}+z_{2}\right)-4 a_{45} v_{3}-a_{53} t_{1}-a_{54} t_{2}+a_{51} t_{3}+a_{52} t_{4}-2 t_{5} .
\end{aligned}
$$

The $t_{j}$ induce translations on $a_{5 j}$, so we can always normalize $\alpha_{5}=0$. This forces $t_{i}=\lambda_{i} v_{3}$, where $\left(\lambda_{1}, \lambda_{2}, \lambda_{3}, \lambda_{4}\right)=\left(-\frac{4}{3} a_{41}, 4 a_{42}, 4 a_{43},-\frac{4}{3} a_{44},-2 a_{45}\right)$. Hence, there exists functions $T_{i j}$ such that

$$
\tau_{i}=\lambda_{i} \nu_{3}+\sum_{j} T_{i j} \varpi_{j}
$$

We have reduced to a 3 -dim structure algebra (with parameters $v_{3}, z_{1}, z_{2}$ ). We will show that:

Theorem 4.1. Any multiply transitive type $N$ structure with the normalizations $A=y^{4}$ and $\alpha_{5}=0$ satisfies $B=C=D=0$.

The integrability conditions $0=d^{2} \nu_{i} \wedge \varpi_{5}(i=1,2,3)$ force

$$
B_{1}=B_{2}=B_{5}=B_{6}=0, \quad a_{41}=-2 B_{4}, \quad a_{42}=-2 B_{3}, \quad a_{43}=+2 B_{7}, \quad a_{44}=-2 B_{8},
$$

and $0=d\left(B_{1}\right)=d\left(B_{2}\right)=d\left(B_{5}\right)=d\left(B_{6}\right)$ are equivalent to:

$$
\beta_{1}=\beta_{5}=0, \quad \beta_{2}=2 B_{3} \alpha_{4}, \quad \beta_{6}=2 B_{7} \alpha_{4}
$$

Moreover, $B_{3}$ and $B_{7}$ are relative invariants:

$$
\begin{array}{ll}
\delta B_{3}=-\left(\frac{7 z_{1}+3 z_{2}}{4}\right) B_{3}, & \delta B_{4}=5 B_{3} v_{3}-\left(\frac{5 z_{1}+z_{2}}{4}\right) B_{4}, \\
\delta B_{7}=-\left(\frac{3 z_{1}+7 z_{2}}{4}\right) B_{7}, & \delta B_{8}=5 B_{7} v_{3}-\left(\frac{z_{1}+5 z_{2}}{4}\right) B_{8} .
\end{array}
$$

\footnotetext{
${ }^{3}$ This normalization is always possible working over $\mathbb{C}$, but over $\mathbb{R}$ we would have two possibilities: $A= \pm y^{4}$.
} 
If $B_{3} B_{7}$ is nowhere vanishing, we can normalize $\left(B_{3}, B_{4}, B_{7}\right)=(1,0,1)$. This trivializes the structure algebra and so such structures admit at most five symmetries (henceforth excluded since these are not multiply transitive). We have the following trichotomy 4 :

\begin{tabular}{cc} 
Condition & Bound on symmetry dimension \\
\hline$B_{3}=B_{7}=0$ & 8 \\
$\left(B_{3}, B_{7}\right) \neq(0,0), B_{3} B_{7}=0$ & 6 \\
$B_{3} B_{7} \neq 0$ & 5
\end{tabular}

Lemma 4.2. No structures with 6 symmetries exist when $\left(B_{3}, B_{7}\right) \neq(0,0), B_{3} B_{7}=0$.

Proof. By duality, take $B_{3} \neq 0$ and $B_{7}=0$. Normalizing $\left(B_{3}, B_{4}\right)=(1,0)$ forces $z_{2}=-\frac{7}{3} z_{1}, v_{3}=0$. The structure algebra is reduced to $\operatorname{diag}\left(\frac{z_{1}}{6},-\frac{7 z_{1}}{6},-\frac{z_{1}}{2}, \frac{3 z_{1}}{2}\right)$, and this acts trivially vertically under the 6 symmetry assumption. Hence, $B_{8}=0$. From $0=d\left(B_{3}\right)=d\left(B_{4}\right)=d\left(B_{7}\right)=d\left(B_{8}\right)$,

$$
\zeta_{2}=-\frac{7}{3} \zeta_{1}+\frac{4}{3} \beta_{3}, \quad \nu_{3}=\frac{1}{5}\left(\tau_{2}-\beta_{4}\right), \quad \beta_{7}=0, \quad \beta_{8}=-\tau_{3} .
$$

All coefficients with nonzero (vertical) scaling weight with respect to $z_{1}$ must vanish. Differentiating the relations on $\zeta_{2}, \nu_{1}, \nu_{2}, \nu_{3}, \tau_{1}, \tau_{2}, \tau_{3}, \tau_{4}, \tau_{5}$, we conclude from these weights that

$$
\beta_{3}=\beta_{4}=\beta_{8}=\tau_{2}=\tau_{3}=\tau_{4}=\nu_{3}=0, \quad a_{45}=0, \quad T_{i j}=0, \quad(i, j) \notin\{(1,5),(5,1)\}
$$

But differentiating $\nu_{3}=0$ then yields the contradiction $0=\varpi_{1} \wedge \varpi_{4}-\left(T_{15}+1\right) \varpi_{2} \wedge \varpi_{5}$.

Thus, $B_{3}=B_{7}=0$ for multiply transitive structures. Now $0=d\left(B_{3}\right)=d\left(B_{7}\right)$ implies:

$$
\beta_{3}=B_{4} \alpha_{4}, \quad \beta_{7}=B_{8} \alpha_{4} .
$$

Moreover, $B_{4}, B_{8}$ are relative invariants.

\begin{tabular}{ccc} 
Case & Condition & Bound on symmetry dimension \\
\hline (a) & $B_{4}=B_{8}=0$ & 8 \\
(b) & $\left(B_{4}, B_{8}\right) \neq(0,0), B_{4} B_{8}=0$ & 7 \\
(c) & $B_{4} B_{8} \neq 0$ & 6
\end{tabular}

Lemma 4.3. Any multiply transitive type $N$ structure with normalization $A=y^{4}$ satisfies $B=0$.

Proof. Suppose $B_{4} B_{8} \neq 0$. Normalizing $B_{4}=B_{8}=1$ forces $z_{1}=z_{2}=0$. Hence, $\zeta_{i}=Z_{i j} \varpi_{j}$. For multiply transitive structures, $Z_{i j}$ are constant, $\varpi_{1}, \ldots, \varpi_{5}, \nu_{3}$ linearly independent, and $v_{3}$ must act vertically trivially. This forces $C_{i}=0(i \neq 3,6,9), D_{1}=D_{3}=0$,

$$
T_{23}=T_{32}=-\frac{16}{9}, \quad T_{22}=T_{33}=-\frac{16}{3}, \quad Z_{12}=-Z_{23}=4, \quad Z_{13}=-Z_{22}=\frac{4}{3}, \quad a_{45}=-\frac{32}{9},
$$

and several more linear relations between $T_{i j}$ and $Z_{i j}$. Since all $B, C, D$ coefficients must be constant, apply $d$ to get further relations. Imposing Bianchi identities yields $C_{3}=C_{9}=\frac{8}{3}$ and $C_{6}=\frac{4}{9}$. A contradiction is then obtained from $0=d^{2} \tau_{1} \wedge \varpi_{3} \wedge \varpi_{4} \neq 0$.

The case $B_{4} \neq 0, B_{8}=0$ is more involved, but similarly yields a contradiction.

Given $B=0$, the conditions $0=d^{2} \nu_{i}$ imply $C_{j}=0$ for $j \neq 3,6,9$, and

$$
a_{45}=C_{6}, \quad b_{42}=-C_{3}, \quad b_{43}=2 C_{6}, \quad b_{82}=-2 C_{6}, \quad b_{83}=C_{9}, \quad b_{81}=-b_{44} .
$$

Now imposing $0=d\left(B_{4}\right)=d\left(B_{8}\right)$ and $0=d\left(\nu_{2}-\alpha_{4}\right)$, we obtain $C_{3}=C_{6}=C_{9}=0$, so $C=0$, and

$$
T_{2 i}=0(i \neq 1), \quad T_{3 j}=0(j \neq 4), \quad T_{21}=b_{41}, \quad T_{34}=-b_{84}, \quad b_{44}=b_{45}=b_{85}=0 .
$$

\footnotetext{
${ }^{4}$ Implicitly, this trichotomy depends on $B_{3}$ and $B_{7}$ have locally constant type, i.e. the stated invariant conditions are true locally. For (multiply) transitive structures, this is always true.
} 
For $i=1, \ldots, 9,0=d\left(C_{i}\right)$ implies $\gamma_{i}=0$. Then $0=d^{2} \tau_{1}=d^{2} \tau_{2}$ implies $D=0$. For $i=1, \ldots, 4$, $0=d\left(D_{i}\right)$ implies $\delta_{i}=0$. Now $0=d\left(\nu_{1}-\frac{1}{4}\left(\zeta_{1}+\zeta_{2}\right)\right)$ implies relations among the $T_{i j}$. We obtain:

$$
\begin{aligned}
& \tau_{2}=T_{21} \varpi_{1}, \quad \tau_{3}=-3 T_{43} \varpi_{4}, \quad \tau_{5}=T_{51} \varpi_{1}+T_{54} \varpi_{4}+T_{55} \varpi_{5}, \\
& \tau_{1}=T_{11} \varpi_{1}-\frac{T_{21}}{3} \varpi_{2}+T_{41} \varpi_{4}+\frac{2}{3} T_{51} \varpi_{5}, \quad \tau_{4}=T_{41} \varpi_{1}+T_{43} \varpi_{3}+T_{44} \varpi_{4}+\frac{2}{3} T_{54} \varpi_{5},
\end{aligned}
$$

and all other $T_{i j}$ not appearing above are zero. Differentiating the $\tau_{i}$-relations $(i=1, \ldots, 4)$, we obtain the vertical action:

$$
\begin{aligned}
& \delta T_{21}=-2 T_{21} z_{1}, \quad \delta T_{11}=\left(\frac{-3 z_{1}+z_{2}}{2}\right) T_{11}+\frac{2}{3} T_{21} v_{3} \\
& \delta T_{43}=-2 T_{43} z_{2}, \quad \delta T_{44}=\left(\frac{z_{1}-3 z_{2}}{2}\right) T_{44}+2 T_{43} v_{3}
\end{aligned}
$$

Lemma 4.4. With normalizations as above, we must have $T_{21}=T_{43}=T_{51}=T_{54}=T_{55}=0$.

Proof. If $T_{21} T_{43} \neq 0$, then there are at most 5 symmetries. If $T_{21} \neq 0$ and $T_{43}=0$, normalize $T_{21}=1$ and $T_{11}=0$, and write $\zeta_{1}=Z_{1 j} \varpi_{j}, \nu_{3}=V_{3 j} \varpi_{j}$. We have at most 6 symmetries, and for 6 the residual structure algebra (generated by $z_{2}$ ) must act vertically trivially. This forces $\zeta_{1}=\nu_{3}=0$ and $T_{41}=T_{44}=T_{51}=T_{54}=T_{55}=0$. But $0=d \zeta_{1}=-2 \varpi_{1} \wedge \varpi_{2} \neq 0$ yields a contradiction. The $T_{43} \neq 0, T_{21}=0$ case similarly yields a contradiction. Thus, we conclude that $T_{21}=T_{43}=0$ and hence $\tau_{2}=\tau_{3}=0$. From $0=d \tau_{2}=d \tau_{3}$, we obtain $T_{51}=T_{54}=T_{55}=0$.

SUMMARY: For multiply transitive type $N$ structures, we have reduced to an 8-dimensional subbundle of the original Cartan bundle (given the normalizations $A=y^{4}$ and $\alpha_{5}=0$ ).

- Curvature coefficients: All $A, B, C, D$ are zero, except $A_{5}=1$.

- Coframe: $\varpi_{1}, \ldots, \varpi_{5}, \zeta_{1}, \zeta_{2}, \nu_{3}$. Relations on other forms:

$$
\nu_{1}=\frac{1}{4}\left(\zeta_{1}+\zeta_{2}\right), \quad \nu_{2}=\tau_{2}=\tau_{3}=\tau_{5}=0, \quad \tau_{1}=T_{11} \varpi_{1}+T_{41} \varpi_{4}, \quad \tau_{4}=T_{41} \varpi_{1}+T_{44} \varpi_{4} .
$$

- Among $\alpha_{i}, \beta_{j}, \gamma_{k}, \delta_{\ell}$, the only possibly nontrivial forms are $\beta_{4}=\tau_{2}$ and $\beta_{8}=-\tau_{3}$.

- All Bianchi identities are satisfied, e.g. $0=d^{2} \nu_{1}$, etc.

- Structure group: $\left[\begin{array}{cccc}r_{1} & 0 & 0 & 0 \\ 0 & r_{2} & 0 & 0 \\ 0 & s & r_{3} & 0 \\ 0 & 0 & 0 & r_{4}\end{array}\right]$, where $r_{1} r_{2} r_{3} r_{4}=1, r_{1} r_{3}^{2}=r_{2}^{2} r_{4}$, i.e. $r_{1}=\frac{1}{r_{2}{ }^{4} r_{4}{ }^{3}}$, $r_{3}=r_{2}{ }^{3} r_{4}{ }^{2}$. This induces $\left\{\begin{array}{l}\tilde{T}_{11}=\frac{r_{2}{ }^{2}}{r_{1}^{2}} T_{11}=r_{2}{ }^{10} r_{4}{ }^{6} T_{11} \\ \tilde{T}_{41}=\frac{r_{2} r_{4}}{r_{1} r_{3}} T_{41}=r_{2}{ }^{2} r_{4}{ }^{2} T_{41} \\ \tilde{T}_{44}=\frac{r_{4}{ }^{2}}{r_{3}{ }^{2}} T_{44}=\frac{1}{r_{2}{ }^{6} r_{4}{ }^{2}} T_{44}\end{array} \Rightarrow\left\{\begin{array}{l}\delta\left(T_{11}\right)=\left(\frac{z_{2}-3 z_{1}}{2}\right) T_{11} \\ \delta\left(T_{41}\right)=-\left(\frac{z_{1}+z_{2}}{2}\right) T_{41} \\ \delta\left(T_{44}\right)=\left(\frac{z_{1}-3 z_{2}}{2}\right) T_{44}\end{array}\right.\right.$

- Let $\Phi$ be the duality transformation:

$$
\begin{aligned}
& \varpi_{1} \leftrightarrow \varpi_{4}, \quad \varpi_{2} \leftrightarrow \varpi_{3}, \quad \varpi_{5} \mapsto-\varpi_{5}, \quad \tau_{1} \leftrightarrow \tau_{4}, \quad \tau_{2} \leftrightarrow \tau_{3}, \quad \tau_{5} \mapsto-\tau_{5}, \\
& \zeta_{1} \leftrightarrow \zeta_{2}, \quad \nu_{1} \text { fixed, } \quad \nu_{2} \mapsto-\nu_{2}, \quad \nu_{3} \mapsto-\nu_{3} .
\end{aligned}
$$

This preserves $A$ and induces $\left(\tau_{1}, \tau_{4}\right) \mapsto\left(\tau_{4}, \tau_{1}\right)$ and so $\left(T_{11}, T_{41}, T_{44}\right) \mapsto\left(T_{44}, T_{41}, T_{11}\right)$.

The case analysis based on the relative invariants $T_{11}, T_{41}, T_{44}$ is straightforward. Table 4.1 summarizes this classification and Table 4.2 contains the structure equations obtained.

Some care is required to deduce any redundancy of parameters appearing in the structure equations. Consider the case $T_{11} \neq 0$. Normalize $T_{11}=1$, so $r_{4}{ }^{6}=\frac{1}{r_{2}{ }^{10}}$, and $\zeta_{2}=3 \zeta_{1}+Z_{21} \varpi_{1}+Z_{24} \varpi_{4}$ is forced. Quotienting the structure group by $\operatorname{diag}\left(r_{2}, r_{2}, r_{2}, r_{2}\right)$ (since these act trivially), we may WLOG take the diagonal to be $\operatorname{diag}\left(\frac{1}{r_{2} r_{4}{ }^{3}}, 1, r_{2}{ }^{2} r_{4}{ }^{2}, \frac{r_{4}}{r_{2}}\right)$. Let $Q$ be the residual group below. 


\begin{tabular}{c||c|c|c} 
& $T_{11}=T_{44}=0$ & $T_{11} \neq 0, T_{44}=0$ & $T_{11} T_{44} \neq 0$ \\
\hline \hline$T_{41}=0$ & N.8 & N.7-1 & N.6-2 \\
$T_{41} \neq 0$ & N.7-2 & none & N.6-1
\end{tabular}

TABLE 4.1. Classification of multiply transitive type $\mathrm{N}$ structures

(1) N.6-1: $T_{41}=1$ and $\zeta_{1}=Z_{11} \varpi_{1}+Z_{14} \varpi_{4}$. Then $Q \cong \mathbb{Z}_{2}$, generated by $E=\operatorname{diag}(-1,1,1,-1)$.

$$
\tilde{T}_{44}=T_{44}, \quad \tilde{Z}_{j 1}=-Z_{j 1}, \quad \tilde{Z}_{j 4}=-Z_{j 4} \quad(j=1,2) .
$$

Let $Z_{24}=4 a$, so $\pm a$ yield the same structure. We must $a\left(a^{2}+1\right) \neq 0$ and

$$
Z_{11}=\frac{1-2 a^{2}}{a}, \quad Z_{14}=\frac{-a\left(2 a^{2}+1\right)}{a^{2}+1}, \quad T_{44}=\frac{a^{4}}{\left(a^{2}+1\right)^{2}}, \quad Z_{21}=\frac{4\left(a^{2}-1\right)}{a} .
$$

Thus, $a^{2} \in \mathbb{C} \backslash\{0,-1\}$ is the essential parameter.

(2) N.6-2: $T_{44}=1$ and $\zeta_{1}=-\frac{3}{8} Z_{21} \varpi_{1}+Z_{14} \varpi_{4}$. Write $Z_{21}=-4 a$ and $Z_{14}=\frac{b}{2}$. Here, $Q \cong \mathbb{Z}_{2} \times \mathbb{Z}_{2}$ generated by $M_{1}=\operatorname{diag}(-1,1,1,-1)$ and $M_{2}=\operatorname{diag}(1,1,-1,1)$. Then:

$$
M_{1}: \quad(\tilde{a}, \tilde{b})=(-a,-b) ; \quad M_{2}: \quad(\tilde{a}, \tilde{b})=(a,-b) .
$$

Thus, $\left(a^{2}, b^{2}\right) \in \mathbb{C} \times \mathbb{C}$ is the essential parameter.

(3) N.7-1: $T_{41}=T_{44}=0, \zeta_{2}=4 a \varpi_{1}+3 \zeta_{1}$. Here, $Q$ has diagonal $\operatorname{diag}\left(\frac{1}{r_{2}{ }^{5} r_{4}{ }^{3}}, 1, r_{2}{ }^{2} r_{4}{ }^{2}, \frac{r_{4}}{r_{2}}\right)$, with $\epsilon:=r_{2}{ }^{5} r_{4}{ }^{3}= \pm 1$. Induced action: $\tilde{a}=\epsilon a$. Thus, $a^{2} \in \mathbb{C}$ is the essential parameter.

\begin{tabular}{|c|c|c|c|}
\hline Model & SD & Structure equations & Embedding into Cartan bundle \\
\hline N.8 & $\checkmark$ & $\begin{array}{l}d \varpi_{1}=\frac{3}{4} \zeta_{1} \wedge \varpi_{1}-\frac{1}{4} \zeta_{2} \wedge \varpi_{1} \\
d \varpi_{2}=\frac{5}{4} \zeta_{1} \wedge \varpi_{2}+\frac{1}{4} \zeta_{2} \wedge \varpi_{2}-\nu_{3} \wedge \varpi_{1} \\
d \varpi_{3}=\frac{1}{4} \zeta_{1} \wedge \varpi_{3}+\frac{5}{4} \zeta_{2} \wedge \varpi_{3}+\nu_{3} \wedge \varpi_{4} \\
d \varpi_{4}=-\frac{1}{4} \zeta_{1} \wedge \varpi_{4}+\frac{3}{4} \zeta_{2} \wedge \varpi_{4} \\
d \varpi_{5}=\zeta_{1} \wedge \varpi_{5}+\zeta_{2} \wedge \varpi_{5}+\varpi_{1} \wedge \varpi_{3}+\varpi_{2} \wedge \varpi_{4} \\
d \nu_{3}=\frac{1}{2} \zeta_{1} \wedge \nu_{3}+\frac{1}{2} \zeta_{2} \wedge \nu_{3}+\varpi_{1} \wedge \varpi_{4} \\
d \zeta_{1}=0 \\
d \zeta_{2}=0\end{array}$ & $\begin{array}{l}\nu_{1}=\frac{1}{4}\left(\zeta_{1}+\zeta_{2}\right) \\
\nu_{2}=0 \\
\tau_{1}=\tau_{2}=\tau_{3}=\tau_{4}=\tau_{5}=0\end{array}$ \\
\hline N.7-1 & $\times$ & $\begin{array}{l}d \varpi_{1}=0 \\
d \varpi_{2}=2 \zeta_{1} \wedge \varpi_{2}-\nu_{3} \wedge \varpi_{1}+a \varpi_{1} \wedge \varpi_{2} \\
d \varpi_{3}=4 \zeta_{1} \wedge \varpi_{3}+\nu_{3} \wedge \varpi_{4}+\varpi_{1} \wedge \varpi_{5}+5 a \varpi_{1} \wedge \varpi_{3} \\
d \varpi_{4}=2 \zeta_{1} \wedge \varpi_{4}+3 a \varpi_{1} \wedge \varpi_{4} \\
d \varpi_{5}=4 \zeta_{1} \wedge \varpi_{5}+\varpi_{1} \wedge \varpi_{3}+\varpi_{2} \wedge \varpi_{4}+4 a \varpi_{1} \wedge \varpi_{5} \\
d \nu_{3}=2 \zeta_{1} \wedge \nu_{3}-2 a \nu_{3} \wedge \varpi_{1}+\varpi_{1} \wedge \varpi_{2}+\varpi_{1} \wedge \varpi_{4} \\
d \zeta_{1}=0\end{array}$ & $\begin{aligned} \zeta_{2} & =3 \zeta_{1}+4 a \varpi_{1} \\
\nu_{1} & =\zeta_{1}+a \varpi_{1} \\
\tau_{1} & =\varpi_{1} \\
\nu_{2} & =\tau_{2}=\tau_{3}=\tau_{4}=\tau_{5}=0 \\
& \left(\text { Parameter: } a^{2} \in \mathbb{C}\right)\end{aligned}$ \\
\hline N.7-2 & $\checkmark$ & $\begin{array}{l}d \varpi_{1}=\zeta_{1} \wedge \varpi_{1} \\
d \varpi_{2}=\zeta_{1} \wedge \varpi_{2}-\nu_{3} \wedge \varpi_{1}-\varpi_{1} \wedge \varpi_{5} \\
d \varpi_{3}=-\zeta_{1} \wedge \varpi_{3}+\nu_{3} \wedge \varpi_{4}+\varpi_{4} \wedge \varpi_{5} \\
d \varpi_{4}=-\zeta_{1} \wedge \varpi_{4} \\
d \varpi_{5}=\varpi_{1} \wedge \varpi_{3}+\varpi_{2} \wedge \varpi_{4} \\
d \nu_{3}=-\varpi_{2} \wedge \varpi_{4}-\varpi_{1} \wedge \varpi_{3}+\varpi_{1} \wedge \varpi_{4} \\
d \zeta_{1}=2 \varpi_{1} \wedge \varpi_{4}\end{array}$ & $\begin{array}{l}\zeta_{2}=-\zeta_{1} \\
\tau_{1}=\varpi_{4} \\
\tau_{4}=\varpi_{1} \\
\nu_{1}=\nu_{2}=\tau_{2}=\tau_{3}=\tau_{5}=0\end{array}$ \\
\hline
\end{tabular}

All type $\mathrm{N}$ structure equations are given in Table 4.2.

TABLE 4.2. Multiply transitive type $\mathrm{N}$ structure equations 
TABLE 4.2. Multiply transitive type $\mathrm{N}$ structure equations (continued)

\begin{tabular}{|c|c|c|c|}
\hline Model & SD & Structure equations & Embedding into Cartan bundle \\
\hline N.6-1 & $\checkmark$ & 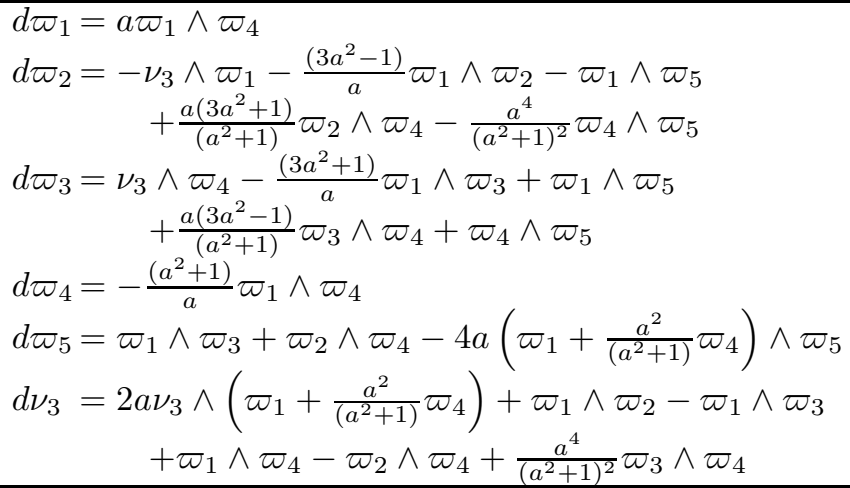 & $\begin{aligned} \zeta_{1} & =-\frac{\left(2 a^{2}-1\right)}{a} \varpi_{1}-\frac{a\left(2 a^{2}+1\right)}{\left(a^{2}+1\right)} \varpi_{4} \\
\zeta_{2} & =-\frac{\left(2 a^{2}+1\right)}{a} \varpi_{1}-\frac{a\left(2 a^{2}-1\right)}{\left(a^{2}+1\right)} \varpi_{4} \\
\nu_{1} & =-a\left(\varpi_{1}+\frac{a^{2}}{\left(a^{2}+1\right)} \varpi_{4}\right) \\
\tau_{1} & =\varpi_{1}+\varpi_{4} \\
\tau_{4} & =\varpi_{1}+\frac{a^{4}}{\left(a^{2}+1\right)^{2}} \varpi_{4} \\
\nu_{2} & =\tau_{2}=\tau_{3}=\tau_{5}=0 \\
& \left(\text { Parameter: } a^{2} \in \mathbb{C} \backslash\{0,-1\}\right)\end{aligned}$ \\
\hline N.6-2 & $\begin{array}{l}\checkmark: \\
a^{2}=b^{2} \\
\times: \\
a^{2} \neq b^{2}\end{array}$ & $\begin{array}{rl}d & d \varpi_{1}=0 \\
d \varpi_{2} & =-\nu_{3} \wedge \varpi_{1}+2 a \varpi_{1} \wedge \varpi_{2}-b \varpi_{2} \wedge \varpi_{4}-\varpi_{4} \wedge \varpi_{5} \\
d \varpi_{3} & =+\nu_{3} \wedge \varpi_{4}+a \varpi_{1} \wedge \varpi_{3}-2 b \varpi_{3} \wedge \varpi_{4}+\varpi_{1} \wedge \varpi_{5} \\
d \varpi_{4} & =0 \\
d \varpi_{5} & =\varpi_{1} \wedge \varpi_{3}+\varpi_{2} \wedge \varpi_{4}+2 a \varpi_{1} \wedge \varpi_{5}+2 b \varpi_{4} \wedge \varpi_{5} \\
d \nu_{3} & =-\nu_{3} \wedge\left(a \varpi_{1}+b \varpi_{4}\right)+\varpi_{1} \wedge \varpi_{2} \\
& \quad+\varpi_{3} \wedge \varpi_{4}+\varpi_{1} \wedge \varpi_{4}\end{array}$ & $\begin{array}{l}\zeta_{1}=\frac{3 a}{2} \varpi_{1}+\frac{b}{2} \varpi_{4} \\
\zeta_{2}=\frac{a}{2} \varpi_{1}+\frac{3 b}{2} \varpi_{4} \\
\nu_{1}=\frac{a}{2} \varpi_{1}+\frac{b}{2} \varpi_{4} \\
\tau_{1}=\varpi_{1}, \tau_{4}=\varpi_{4} \\
\nu_{2}=\tau_{2}=\tau_{3}=\tau_{5}=0 \\
\left(\text { Parameter: }\left(a^{2}, b^{2}\right) \in \mathbb{C} \times \mathbb{C}\right)\end{array}$ \\
\hline
\end{tabular}

4.2. Type $D$ reduction. Normalize $A=6 x^{2} y^{2}$, i.e. $A_{3}=1, A_{5}=A_{4}=A_{2}=A_{1}=0$. Now $0=d\left(A_{i}\right)$ implies:

$$
\nu_{2}=\frac{1}{3} \alpha_{2}, \quad \nu_{3}=\frac{1}{3} \alpha_{4}, \quad \zeta_{2}=-\zeta_{1}+\alpha_{3}, \quad \alpha_{1}=\alpha_{5}=0 .
$$

Differentiating the $\zeta_{2}$-relation above yields the vertical action on coefficients in $\alpha_{3}=a_{3 j} \varpi_{j}$ :

$$
\begin{array}{ll}
\delta a_{31}=a_{31}\left(v_{1}-z_{1}\right)-t_{1}, \quad & \delta a_{32}=-a_{32}\left(v_{1}+z_{1}\right)-t_{2}, \quad \delta a_{33}=a_{33}\left(-v_{1}+z_{1}\right)-t_{3}, \\
\delta a_{34}=a_{34}\left(v_{1}+z_{1}\right)-t_{4}, & \delta a_{35}=a_{32} t_{4}+a_{31} t_{3}-a_{34} t_{2}-a_{33} t_{1}-2 t_{5} .
\end{array}
$$

Normalize $\alpha_{3}=0$, so $\tau_{i}=T_{i j} \varpi_{j}$. We have reduced to the 2-dimensional structure algebra $\operatorname{diag}\left(\frac{z_{1}}{2}, v_{1}-\frac{z_{1}}{2},-v_{1}-\frac{z_{1}}{2}, \frac{z_{1}}{2}\right)$, so all type D structures admit at most seven symmetries. Using duality and the $G_{0}$-map $(x, y) \mapsto(y,-x)$, we can assume that $B_{1}$ or $B_{2}$ is nonzero, or $B=0$.

For the 7-symmetry case, the 2-dimensional structure algebra must act trivially. This forces:

- only $C_{5}$ (necessarily constant) to survive among $B, C, D$ coefficients;

- $\alpha_{2}=\alpha_{4}=0\left(\right.$ so $\left.\nu_{2}=\nu_{3}=0\right)$;

- all $T_{i j}$ to vanish except $T_{13}, T_{24}, T_{31}, T_{42}, T_{55}$ (necessarily constants).

From $0=d \nu_{2}=d\left(\zeta_{1}+\zeta_{2}\right)=d\left(\tau_{1}-T_{13} \varpi_{3}\right)=d\left(\tau_{2}-T_{24} \varpi_{4}\right)$, we obtain

$$
T_{31}=T_{13}=C_{5}-\frac{1}{2}, \quad T_{42}=T_{24}=-C_{5}-\frac{1}{2}, \quad T_{55}=\left(C_{5}\right)^{2}+\frac{1}{4} .
$$

Now, $0=d\left(B_{i}\right)=d\left(C_{j}\right)=d\left(D_{k}\right)$ forces $\beta_{i}=0(i=1,4,5,8)$, and $\gamma_{j}=0$, and

$$
\left(\beta_{2}, \beta_{3}, \beta_{6}, \beta_{7}\right)=\left(\tau_{2}, \tau_{1},-\tau_{3}, \tau_{4}\right), \quad\left(\delta_{1}, \delta_{2}, \delta_{3}, \delta_{4}\right)=2 C_{5}\left(\beta_{2}, \beta_{3}, \beta_{6}, \beta_{7}\right) .
$$

This yields model D.7. The 2-dimensional structure group is generated by $\operatorname{diag}\left(r_{1}, \frac{r_{2}}{r_{1}}, \frac{1}{r_{1} r_{2}}, r_{1}\right)$ $\left(r_{1}, r_{2} \in \mathbb{C}^{\times}\right)$, along with $\operatorname{diag}\left(e^{i \pi / 4}, e^{i \pi / 4},-e^{i \pi / 4}, e^{i \pi / 4}\right)$ and $\operatorname{diag}\left(1,\left[\begin{array}{cc}0 & 1 \\ -1 & 0\end{array}\right], 1\right)$.5 Only this last transformation acts non-trivially on $C_{5}$, i.e. $C_{5} \mapsto-C_{5}$. Thus, $\left(C_{5}\right)^{2} \in \mathbb{C}$ is the essential parameter.

\footnotetext{
${ }^{5}$ The latter two correspond to $(x, y) \mapsto(x,-y)$ and $(x, y) \mapsto(-y, x)$.
} 
The 6-symmetry case proceeds similarly, but is very tedious, particularly for the $B_{2} \neq 0$ case that leads to model D.6-2. All type D structure equations are given in Table 4.3.

TABLE 4.3. Multiply transitive type $\mathrm{D}$ structure equations

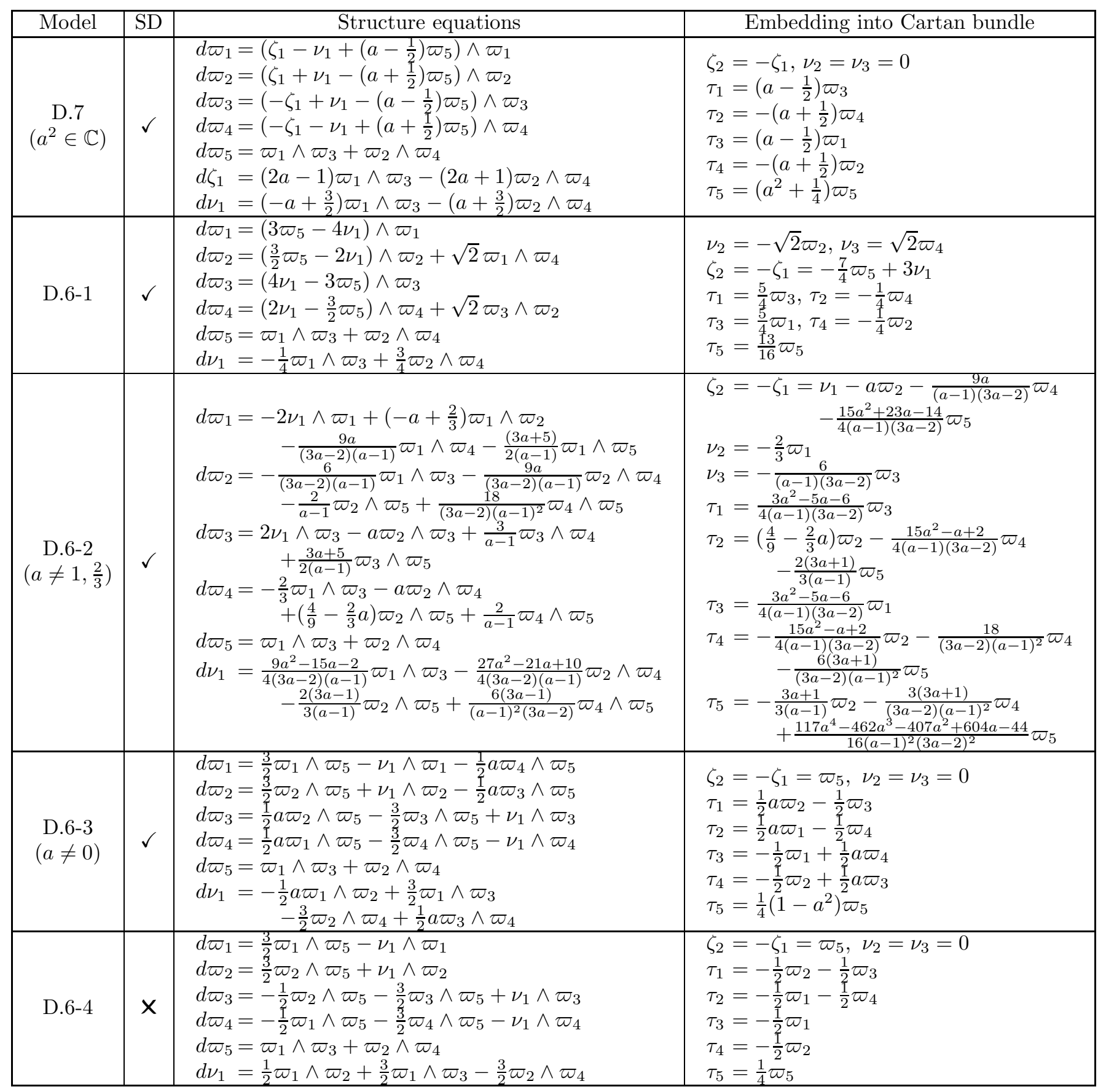

4.3. Type III reduction. Normalize $A=x y^{3}$, i.e. $A_{4}=\frac{1}{4}, A_{5}=A_{3}=A_{2}=A_{1}=0$. Then $0=d\left(A_{i}\right)$ implies:

$$
\alpha_{1}=\alpha_{2}=0, \quad \nu_{2}=2 \alpha_{3}, \quad \nu_{1}=\frac{1}{2}\left(\zeta_{1}+\zeta_{2}\right)-2 \alpha_{4}, \quad \nu_{3}=\alpha_{5}
$$


Differentiating the $\nu_{i}$-relations above yields

$$
\begin{aligned}
& 2 \delta a_{31}=-\left(3 z_{1}+z_{2}\right) a_{31}+t_{2}, \quad 2 \delta a_{34}=-\left(z_{1}+3 z_{2}\right) a_{34}-t_{3}, \\
& 2 \delta a_{41}=\left(z_{2}-z_{1}\right) a_{41}-t_{1}, \quad 2 \delta a_{44}=\left(z_{1}-z_{2}\right) a_{44}-t_{4} .
\end{aligned}
$$

Normalize $a_{31}=a_{34}=a_{41}=a_{44}=0\left(\right.$ so $\left.t_{1}=t_{2}=t_{3}=t_{4}=0\right)$. Then $2 \delta a_{45}=-2\left(z_{1}+z_{2}\right) a_{45}-t_{5}$, so normalize $a_{45}=0$, and let $\tau_{i}=T_{i j} \varpi_{j}$. We have reduced to a 7 -dimensional subbundle with:

$$
\begin{array}{lll}
\delta a_{42}=-\left(\frac{3 z_{1}+z_{2}}{2}\right) a_{42} & \delta a_{51}=\left(\frac{z_{1}+3 z_{2}}{2}\right) a_{51} \\
\delta a_{43}=-\left(\frac{z_{1}+3 z_{2}}{2}\right) a_{43} & \delta a_{52}=\left(\frac{z_{2}-z_{1}}{2}\right) a_{52} \\
\delta a_{32}=-\left(\frac{5 z_{1}+3 z_{2}}{2}\right) a_{32}, & \delta a_{53}=\left(\frac{z_{1}-z_{2}}{2}\right) a_{53} \\
\delta a_{33}=-\left(\frac{3 z_{1}+5 z_{2}}{2}\right) a_{33} & \delta a_{54}=\left(\frac{3 z_{1}+z_{2}}{2}\right) a_{54} \\
\delta a_{35}=-2\left(z_{1}+z_{2}\right) a_{35} & \delta a_{55}=0
\end{array}
$$

However, as indicated in Theorem 3.1, there are no type III structures with 7 symmetries.

Now, $0=d^{2} \nu_{2} \wedge \varpi_{45}=d^{2} \nu_{2} \wedge \varpi_{15}$ implies $B_{1}=B_{5}=0$, and $0=d\left(B_{1}\right)=d\left(B_{5}\right)$ is equivalent to:

$$
\beta_{1}=6 B_{2} \alpha_{3}, \quad \beta_{5}=6 B_{6} \alpha_{3},
$$

and further Bianchi identities imply

$$
a_{42}=-2 B_{3}, \quad a_{43}=2 B_{7}, \quad a_{52}=-2 B_{4}, \quad a_{32}=-2 B_{2}, \quad a_{33}=2 B_{6}, \quad a_{53}=2 B_{8}, \quad B_{2} B_{6}=0 .
$$

There is a duality inducing $\left(B_{j}, B_{j+4}\right) \mapsto\left(-B_{j+4}, B_{j}\right)$, where $j=1, \ldots, 4$, so WLOG, we may assume that one of $B_{2}, B_{3}, B_{4}$ is nonzero, or $B=0$. Similar calculations show that for multiply transitive structures, we must have $B_{2}=B_{3}=0$ (hence, $B_{6}=B_{7}=0$ also). Up to duality, we only have: III.6-1 $\left(B_{4} \neq 0\right.$ branch), and III.6-2 $(B=0$ branch). Structure equations are given in Table 4.4 .

TABLE 4.4. Multiply transitive type III structure equations

\begin{tabular}{|c|l|l|l|}
\hline Model & SD & \multicolumn{1}{|c|}{ Structure equations } & Embedding into Cartan bundle \\
\hline & & $d \varpi_{1}=\varpi_{1} \wedge \varpi_{4}$ & \\
& & $d \varpi_{2}=\left(-\frac{5}{4} \varpi_{1}+\varpi_{4}+2 \zeta_{1}\right) \wedge \varpi_{2}-\frac{1}{2} \varpi_{1} \wedge \varpi_{5}$ & $\zeta_{2}=-\varpi_{1}+2 \varpi_{4}+\zeta_{1}$ \\
& $d \varpi_{3}=-\frac{3}{2} \varpi_{1} \wedge \varpi_{3}+\frac{3}{16} \varpi_{1} \wedge \varpi_{5}-\frac{3}{4} \varpi_{2} \wedge \varpi_{4}$ & $\nu_{1}=\zeta_{1}-\frac{1}{2} \varpi_{1}+\varpi_{4}$ \\
III.6-1 & $\times$ & $-3 \varpi_{3} \wedge \varpi_{4}-2 \varpi_{3} \wedge \zeta_{1}+\frac{3}{4} \varpi_{4} \wedge \varpi_{5}$ & $\nu_{3}=-\frac{3}{4} \varpi_{2}-\frac{1}{8} \varpi_{5}$ \\
& & $d \varpi_{4}=-\frac{1}{2} \varpi_{1} \wedge \varpi_{4}$ & $\tau_{1}=\frac{3}{16} \varpi_{1}+\frac{5}{8} \varpi_{4}$ \\
& & $d \varpi_{5}=\varpi_{1} \wedge \varpi_{3}+\varpi_{2} \wedge \varpi_{4}-\varpi_{1} \wedge \varpi_{5}$ & $\tau_{4}=\frac{3}{8} \varpi_{1}$ \\
& & $+2 \varpi_{4} \wedge \varpi_{5}-2 \varpi_{5} \wedge \zeta_{1}$ & $\nu_{2}=\tau_{2}=\tau_{3}=\tau_{5}=0$ \\
& & $d \zeta_{1}=\frac{9}{8} \varpi_{1} \wedge \varpi_{4}$ & \\
\hline \multirow{5}{*}{ III.6-2 } & $\times$ & $d \varpi_{1}=\left(-2 \zeta_{2}+2 \varpi_{3}\right) \wedge \varpi_{1}$ & $\zeta_{1}=4 \varpi_{3}-3 \zeta_{2}$ \\
& & $d \varpi_{2}=\left(-4 \zeta_{2}+6 \varpi_{3}\right) \wedge \varpi_{2}+\frac{1}{4} \varpi_{1} \wedge \varpi_{5}$ & $\nu_{1}=2 \varpi_{3}-\zeta_{2}$ \\
& $d \varpi_{3}=\frac{3}{8} \varpi_{1} \wedge \varpi_{4}$ & $\nu_{2}=0, \nu_{3}=\frac{3}{8} \varpi_{1}-\frac{1}{8} \varpi_{5}$ \\
& $d \varpi_{4}=\left(2 \zeta_{2}-2 \varpi_{3}\right) \wedge \varpi_{4}$, & $\tau_{1}=-\frac{1}{8} \varpi_{4}$ \\
& $d \varpi_{5}=\left(-2 \zeta_{2}+4 \varpi_{3}\right) \wedge \varpi_{5}+\varpi_{1} \wedge \varpi_{3}+\varpi_{2} \wedge \varpi_{4}$ & $\tau_{2}=\tau_{3}=\tau_{5}=0$ \\
& $d \zeta_{2}=\frac{5}{8} \varpi_{1} \wedge \varpi_{4}$ & $\tau_{4}=-\frac{3}{8} \varpi_{1}$ \\
\hline
\end{tabular}

\section{INTEGRATION OF STRUCTURE EQUATIONS}

In this section, we outline the transition from structure equations found in the previous section to the corresponding systems of 2nd order PDEs. This is done in three steps:

(1) Normalize the algebraic structure of the Lie algebra data defined by the structure equations. This step consists of identifying the type of the Lie algebra $\mathfrak{g}$, the isotropy subalgebra $\mathfrak{k}$ and 
the subspaces $E, V \subset \mathfrak{g} / \mathfrak{k}$ corresponding to the two Legendrian subbundles. We note that both $E+\mathfrak{k}$ and $V+\mathfrak{k}$ are in fact subalgebras of $\mathfrak{g}$, as we deal only with integrable structures. We also try to find a good basis in $\mathfrak{g}$, adjusting it to the Levi decomposition and the nilradical. (2) Realize $\mathfrak{g}$ as a transitive Lie algebra of vector fields on $\mathbb{C}^{3}$ in such a way that its isotropy subalgebra at a certain point is equal exactly to $V+\mathfrak{k}$. This guarantees that the first prolongation of $\mathfrak{g}$ is transitive on $J^{1}\left(\mathbb{C}^{2}, \mathbb{C}\right)$ and has isotropy $\mathfrak{k}$ at a certain point.

(3) Finally, we compute all complete systems of 2 nd order PDEs admitting $\mathfrak{g}$ as its symmetry and identify those which correspond to $E+\mathfrak{k}$. In fact, in all cases but one (D.6- $3_{\infty}$, see Example 5.2 below) there is exactly one such system, and this identification is obtained automatically.

Example 5.1. Consider the structure equations for the model D.7 as given in Table 4.3. Simple analysis shows the corresponding Lie algebra $\mathfrak{g}$ has radical of dimension 1 if $a \neq \pm \frac{3}{4}$, and of dimension 4 if $a= \pm \frac{3}{4}$. (Note that $a$ and $-a$ yield equivalent models.) Consider first the case $a \neq \frac{3}{4}$. It is clear that $\mathfrak{g}$ has a 6 -dimensional Levi subalgebra, which is isomorphic to $\mathfrak{s l}_{2}(\mathbb{C}) \times \mathfrak{s l}_{2}(\mathbb{C})$ (the only complex semisimple Lie algebra in this dimension). As any action of this Levi subalgebra on the 1-dimensional radical is trivial, $\mathfrak{g}$ is isomorphic to $\mathfrak{s l}_{2}(\mathbb{C}) \times \mathfrak{s l}_{2}(\mathbb{C}) \times \mathbb{C}$. The corresponding basis change from the Cartan reduced basis to the adapted Lie algebra basis in given in Table A.4.

Next, analyzing the Cartan basis, we see that the isotropy $\mathfrak{k}$ is 2-dimensional and abelian. Moreover, its projection to each $\mathfrak{s l}_{2}(\mathbb{C})$-factor is one-dimensional and diagonalizable, while the intersection with each $\mathfrak{s l}_{2}(\mathbb{C})$-factor is trivial. This implies that $\mathfrak{k}$ is conjugate to the following subalgebra in $\mathfrak{g}$ :

$$
\mathfrak{k} \sim\left\langle H_{1}-Z, H_{2}-\lambda Z\right\rangle, \quad \lambda \in \mathbb{C} \backslash\{0\},
$$

where $H_{1}, H_{2}$ are parts of the standard $\mathfrak{s l}_{2}(\mathbb{C})$-basis $\left\{X_{i}, H_{i}, Y_{i}\right\}$ in each copy of $\mathfrak{s l}_{2}(\mathbb{C})$, and $Z$ spans the center $\mathfrak{z}=\mathbb{C}$. Also, $\lambda=\frac{3+4 a}{3-4 a}$, and the redundancy $a \mapsto-a$ induces the redundancy $\lambda \mapsto \frac{1}{\lambda}$.

Further, it is easy to check that the projections of both $E+\mathfrak{k}$ and $V+\mathfrak{k}$ to each $\mathfrak{s l}_{2}(\mathbb{C})$-factor is two-dimensional. Thus, we can assume that:

$$
\begin{aligned}
& V+\mathfrak{k}=\left\langle X_{1}, X_{2}, H_{1}-Z, H_{2}-\lambda Z\right\rangle, \\
& E+\mathfrak{k}=\left\langle Y_{1}, Y_{2}, H_{1}-Z, H_{2}-\lambda Z\right\rangle .
\end{aligned}
$$

Let us now realize $\mathfrak{g}$ as a Lie algebra of vector fields on $\mathbb{C}^{3}=J^{0}\left(\mathbb{C}^{2}, \mathbb{C}\right)$ with the isotropy subalgebra equal to $V+\mathfrak{k}$. Note that $\mathfrak{h}=V+\mathfrak{k}+\mathfrak{z}$ is a subalgebra of codimension 2 in $\mathfrak{g}$. However, it is not effective, and the maximal ideal of $\mathfrak{g}$ contained in $\mathfrak{h}$ is exactly $\mathfrak{z}$. So, $\mathfrak{g} / \mathfrak{z}$ can be realized as a Lie algebra of vector fields on $\mathbb{C}^{2}$ with the isotropy $\mathfrak{h} / \mathfrak{z}$. But $\mathfrak{g} / \mathfrak{z}$ is isomorphic to $\mathfrak{s l}_{2}(\mathbb{C}) \times \mathfrak{s l}_{2}(\mathbb{C})$ with $\mathfrak{h} / \mathfrak{z}$ identified with the direct product of two subalgebras of upper-triangular matrices. It is easy to see that it integrates to the global action of $\mathrm{PSL}_{2}(\mathbb{C}) \times \mathrm{PSL}_{2}(\mathbb{C})$ on $\mathbb{P}^{1} \times \mathbb{P}^{1}$. Locally this leads to the following realization of $\mathfrak{g} / \mathfrak{z}$ :

$$
\left\langle\partial_{x}, 2 x \partial_{x}, x^{2} \partial_{x}, \partial_{y}, 2 y \partial_{y}, y^{2} \partial_{y}\right\rangle
$$

We can always assume that the realization of $\mathfrak{g}$ is adapted to it. In other words, it can be obtained from the above one by adding terms of the form $f(x, y, u) \partial_{u}$ to the above vector fields and realizing the center $Z$ as a vector field of the form $g(x, y, u) \partial_{u}$. Simple computation shows that we can always adapt the coordinates $(x, y, u)$ such that $Z$ becomes equal to $\partial_{u}$, and we get the following realization of $\mathfrak{g}$ :

$$
\left\langle\partial_{x}, 2 x \partial_{x}+\partial_{u}, x^{2} \partial_{x}+x \partial_{u}, \partial_{y}, 2 y \partial_{y}+\frac{1}{\lambda} \partial_{u}, y^{2} \partial_{y}+\frac{1}{\lambda} y \partial_{u}, \partial_{u}\right\rangle
$$

Prolonging this Lie algebra of vector fields to $J^{1}\left(\mathbb{C}^{2}, \mathbb{C}\right)$ and checking which complete systems of 2nd order PDEs are invariant with respect to it, we immediately get that the only such system has the form:

$$
u_{11}=p^{2}, \quad u_{12}=0, \quad u_{22}=\lambda q^{2} .
$$


Setting now the parameter $\lambda$ to 0 and computing the symmetry algebra of the above system of PDEs, we obtain exactly the Lie algebra $\mathfrak{g}$, its subalgebra $\mathfrak{k}$ and subspaces $E, V \subset \mathfrak{g} / \mathfrak{k}$, that match the exceptional case $a= \pm \frac{3}{4}$ of the Cartan structure equations in case of D.7.

Example 5.2. Consider now the case D.6-3. We note that in this case the Lie algebra $\mathfrak{g}$ defined by the structure equations is semisimple if $a \neq \pm 3$ and has a 3-dimensional abelian ideal otherwise. First, consider the generic case of $a \neq \pm 3$. Then $\mathfrak{g}$ is isomorphic to $\mathfrak{s l}_{2}(\mathbb{C}) \times \mathfrak{s l}_{2}(\mathbb{C})$. As above, denote by $\left\{X_{i}, H_{i}, Y_{i}\right\}, i=1,2$, the standard bases of these two copies of $\mathfrak{s l}_{2}(\mathbb{C})$. Direct inspection of the Cartan structure equations shows that $\mathfrak{k}=\left\langle H_{1}-H_{2}\right\rangle$ and both subalgebras $E+\mathfrak{k}$ and $V+\mathfrak{k}$ are three-dimensional semisimple. But any simple subalgebra of $\mathfrak{g}$ containing $\mathfrak{k}$ has the form:

$$
\left\langle X_{1}+\mu Y_{2}, X_{2}+\mu Y_{1}, H_{1}-H_{2}\right\rangle, \quad \mu \neq 0,
$$

and any two such subalgebras are conjugate to each other by means of inner automorphisms preserving $\mathfrak{k}$. Hence, we can assume that $V+\mathfrak{k}$ corresponds to $\mu=1$, which is exactly the diagonal of the direct product of $\mathfrak{s l}_{2}(\mathbb{C}) \times \mathfrak{s l}_{2}(\mathbb{C})$. Under the classical isomorphism $\mathfrak{s o}(4, \mathbb{C}) \simeq \mathfrak{s l}_{2}(\mathbb{C}) \times \mathfrak{s l}_{2}(\mathbb{C})$ this subalgebra corresponds to the standard embedding of $\mathfrak{s o}(3, \mathbb{C}) \subset \mathfrak{s o}(4, \mathbb{C})$. So, we can realize the Lie algebra $\mathfrak{g}$ as a Lie algebra of vector fields corresponding to the action of $S O(4, \mathbb{C})$ on the three-dimensional complex sphere. In an appropriate coordinate system we get the following vector fields:

$$
\begin{array}{lll}
X_{1}=\partial_{x}, & H_{1}=-2 x \partial_{x}-2 u \partial_{u}, & Y_{1}=-x^{2} \partial_{x}-u \partial_{y}-2 x u \partial_{u} \\
X_{2}=\partial_{y}, & H_{2}=-2 y \partial_{y}-2 u \partial_{u}, & Y_{2}=-y^{2} \partial_{y}-u \partial_{x}-2 y u \partial_{u} .
\end{array}
$$

Again, prolonging this Lie algebra of vector fields to $J^{1}\left(\mathbb{C}^{2}, \mathbb{C}\right)$ and computing all invariant systems of 2nd order PDEs, we obtain the following family of systems:

$$
u_{11}=\lambda p^{2} \frac{\sqrt{u-p q}}{u^{3 / 2}}, \quad u_{12}=1+\lambda(p q-2 u) \frac{\sqrt{u-p q}}{u^{3 / 2}}, \quad u_{22}=\lambda q^{2} \frac{\sqrt{u-p q}}{u^{3 / 2}} .
$$

Each such system corresponds to the subalgebra (5.1) with $\mu=\frac{2 \lambda-1}{2 \lambda+1}$.

In the limiting case of $a= \pm 3$ in the structure equations we get $\mathfrak{g} \simeq \mathfrak{s o}(3, \mathbb{C}) \wedge \mathbb{C}^{3}$ and $\mathfrak{k}+V=\mathbb{C}^{3}$. This pair corresponds to the group of complex Euclidean transformations of $\mathbb{C}^{3}$, which preserves the following family of complete systems of 2nd order PDEs:

$$
u_{11}=\lambda p^{2} \sqrt{1-2 p q}, \quad u_{12}=\lambda(p q-1) \sqrt{1-2 p q}, \quad u_{22}=\lambda q^{2} \sqrt{1-2 p q} .
$$

If $\lambda=0$, this system is flat and has 15-dimensional symmetry algebra. If $\lambda \neq 0$, then we can normalize it to $\lambda=1$ by means of the transformation $(x, y, u) \mapsto(\lambda x, \lambda y, \lambda u)$. To distinguish this special case from the generic one, we denote it by D. $6-3_{\infty}$.

Example 5.3. Consider the case N.6-2, which involves two parameters. The Lie algebra $\mathfrak{g}$ is solvable in this case and has a 4 -dimensional abelian nilradical $\mathfrak{n}$. Two basis elements complementary to $\mathfrak{n}$ act on $\mathfrak{n}$ by the following two commuting matrices:

$$
\left(\begin{array}{cccc}
2 b & 0 & 0 & 1 \\
0 & b & 1 & 0 \\
0 & 1 & 2 b & 0 \\
1 & 0 & 0 & b
\end{array}\right), \quad\left(\begin{array}{cccc}
a & 0 & -1 & 0 \\
0 & 2 a & 0 & -1 \\
-1 & 0 & 2 a & 0 \\
0 & -1 & 0 & a
\end{array}\right) .
$$

If parameters $a, b$ of the structure equations satisfy $a^{2}+4 \neq 0, b^{2}+4 \neq 0$, then both matrices simultaneously diagonalize in a certain basis $\left\{N_{1}, N_{2}, N_{3}, N_{4}\right\}$ of $\mathfrak{n}$ to become:

$$
\begin{aligned}
& \frac{1}{2} \operatorname{diag}\left(3 b-\sqrt{b^{2}+4}, 3 b+\sqrt{b^{2}+4}, 3 b+\sqrt{b^{2}+4}, 3 b-\sqrt{b^{2}+4}\right), \\
& \frac{1}{2} \operatorname{diag}\left(3 a-\sqrt{a^{2}+4}, 3 a-\sqrt{a^{2}+4}, 3 a+\sqrt{a^{2}+4}, 3 a+\sqrt{a^{2}+4}\right) .
\end{aligned}
$$


After rescaling, we can bring them to the form:

$$
\begin{array}{ll}
\operatorname{diag}(\mu-1, \mu, \mu, \mu-1), & \mu=\frac{1}{2}+\frac{3 b}{2 \sqrt{b^{4}+4}}, \\
\operatorname{diag}(\kappa+1, \kappa+1, \kappa+2, \kappa+2), & \kappa=\frac{3}{2}+\frac{3 a}{2 \sqrt{a^{2}+4}} .
\end{array}
$$

Denote by $S_{1}, S_{2}$ the corresponding elements in $\mathfrak{g}$, which span the complementary subspace to $\mathfrak{n}$. In general, this subspace is not a subalgebra, and $\left[S_{1}, S_{2}\right] \in \mathfrak{n}$. But if any of these two matrices is invertible (meaning $\mu \neq 0,1$ or $\kappa \neq-1,-2$ ) then we can always adjust $S_{1}, S_{2}$ by adding elements from $\mathfrak{n}$ such that we get $\left[S_{1}, S_{2}\right]=0$. We note that there are elements $u_{1}, u_{2} \in \mathfrak{n}$ such that $S_{1}+u_{1} \in V+\mathfrak{k}, S_{2}+u_{2} \in E+\mathfrak{k}$.

It is easy to check that the intersection of $V+\mathfrak{k}$ with $\mathfrak{n}$ is two-dimensional and can be made equal to $\left\langle N_{1}-N_{4}, N_{2}-N_{3}\right\rangle$ after suitable rescaling to basis vectors $\left\{N_{i}\right\}$. Hence, in any realization of $\mathfrak{g}$ as a transitive Lie algebra of vector fields on $\mathbb{C}^{3}$ having $V+\mathfrak{k}$ as a stabilizer, $\mathfrak{n}$ will be a 4-dimensional abelian Lie algebra with 2-dimensional orbits. In particular, we can always choose a local coordinate system $(x, y, u)$ in such a way that $N_{3}=\partial_{u}, N_{4}=\partial_{y}$, and two other basis vectors $N_{1}, N_{2}$ will be of the form $f(x) \partial_{y}+g(x) \partial_{u}$. As $S_{1}, S_{2}$ act by scalings on any of $N_{i}, i=1, \ldots, 4$, it is natural to assume that they are represented as linear combinations of vector fields $x \partial_{x}, y \partial_{y}, u \partial_{u}$. Using this ansatz, we immediately get the following representation of $\mathfrak{g}$ :

$$
\begin{aligned}
& S_{1}=-(\mu-1) y \partial_{y}-\mu u \partial_{u}, \\
& S_{2}=-x \partial_{x}-(\kappa+2) y \partial_{y}-(\kappa+2) u \partial_{u}, \\
& N_{1}=x \partial_{y}, \quad N_{2}=x \partial_{u}, \quad N_{3}=\partial_{u}, \quad N_{4}=\partial_{y} .
\end{aligned}
$$

Prolonging this Lie algebra of vector fields to $J^{1}\left(\mathbb{C}^{2}, \mathbb{C}\right)$ and computing all invariant complete systems of 2 nd order PDEs, we arrive at the following system:

$$
u_{11}=q^{\mu} x^{\kappa}, \quad u_{12}=0, \quad u_{22}=0
$$

The special values of parameters we omitted on the way can be treated in a similar way and lead to the following systems of PDEs:

- $a^{2}+4=0, b^{2}+4 \neq 0$ (or equivalently, $a^{2}+4 \neq 0, b^{2}+4=0$ ):

$$
u_{11}=e^{q} x^{\kappa}, \quad u_{12}=0, \quad u_{22}=0 .
$$

- $a^{2}+4=b^{2}+4=0$ :

$$
u_{11}=e^{q} e^{x}, \quad u_{12}=0, \quad u_{22}=0 .
$$

- $\mu=0,1$ (or equivalently, $\kappa=-1,-2$ ):

$$
u_{11}=\ln (q) x^{\kappa}, \quad u_{12}=0, \quad u_{22}=0 .
$$

More details on restrictions on parameters and realizations of $\mathfrak{g}$ in terms of vector fields for these special values of parameters are given in Table A.1.

\section{REFERENCES}

1. R.J. Baston and M.G. Eastwood, The Penrose transform: its interaction with representation theory, Oxford Mathematical Monographs, Clarendon Press, Oxford, 1989.

2. G. Bol, Über topologische Invarianten von zwei Kurvenscharen in Raum, Abhandlungen Math. Sem. Univ. Hamburg., 9 (1932), no. 1, 15-47.

3. A. Čap, Correspondence spaces and twistor spaces for parabolic geometries, J. reine angew. Math. 582 (2005), $143-172$.

4. A. Čap and J. Slovák, Parabolic Geometries I: Background and General Theory, Mathematical Surveys and Monographs, vol. 154, American Mathematical Society, 2009. 
5. É. Cartan, Les systèmes de Pfaff à cinq variables et les équations aux dérivées partielles du second ordre, Ann. Sci. Éc. Norm. Supér. (3) 27 (1910), 109-192.

6. É. Cartan, Sur les variétes à connexion projective, Bull. Soc. Math. France, 52 (1924), 205-241.

7. É. Cartan, Sur la géométrie pseudo-conforme des hypersurfaces de l'espace de deux variables complexes, Ann. Mat. Pura Appl., IV. Ser. 11 (1932), 17-90.

8. É. Cartan, Sur la géométrie pseudo-conforme des hypersurfaces de l'espace de deux variables complexes II, Ann. Sc. Norm. Super. Pisa, II. Ser. 1 (1932), no. 4, 333-354.

9. B. Doubrov, A. Govorov, A new example of a generic 2-distribution on a 5-manifold with large symmetry algebra, arXiv:1305.7297 (2013).

10. R.B. Gardner, The method of equivalence and its applications, CBMS-NSF Regional Conference Series in Applied Mathematics, 58. Society for Industrial and Applied Mathematics (SIAM), Philadelphia, PA, 1989.

11. B. Kostant, Lie algebra cohomology and the generalized Borel-Weil theorem, Ann. Math. 74, no. 2 (1961), 329-387.

12. B. Kruglikov, D. The, The gap phenomenon in parabolic geometries, J. reine angew. Math. (2014), doi: 10.1515/crelle-2014-0072.

13. A.V. Loboda, Homogeneous real hypersurfaces in $\mathbb{C}^{3}$ with two-dimensional isotropy groups, Tr. Mat. Inst. Steklova 235 (2001), Anal. i Geom. Vopr. Kompleks. Analiza, 114-142; (Russian) translation in Proc. Steklov Inst. Math. 2001, no. 4 (235), 107-135.

14. A.V. Loboda, Homogeneous strictly pseudoconvex hypersurfaces in $\mathbb{C}^{3}$ with two-dimensional isotropy groups, Mat. Sb. 192 (2001), no. 12, 3-24; (Russian) translation in Sb. Math. 192 (2001), no. 11-12, 1741-1761.

15. P.J. Olver, Symmetry, invariants, and equivalence, Springer-Verlag, New York, 1995.

16. M. Takeuchi, Lagrangean contact structures on projective cotangent bundles. Osaka J. Math. 31 (1994), no. 4, 837-860.

17. A. Tresse, Détermination des invariants ponctuels de l'équation différentielle ordinaire du second ordre $y^{\prime \prime}=$ $\omega\left(x, y, y^{\prime}\right)$, Leipzig. 87 S. gr. $8^{\circ}$. (1896).

\section{Appendix A. Classification Tables}


Table A.1. Classification of type $\mathrm{N}$ cases

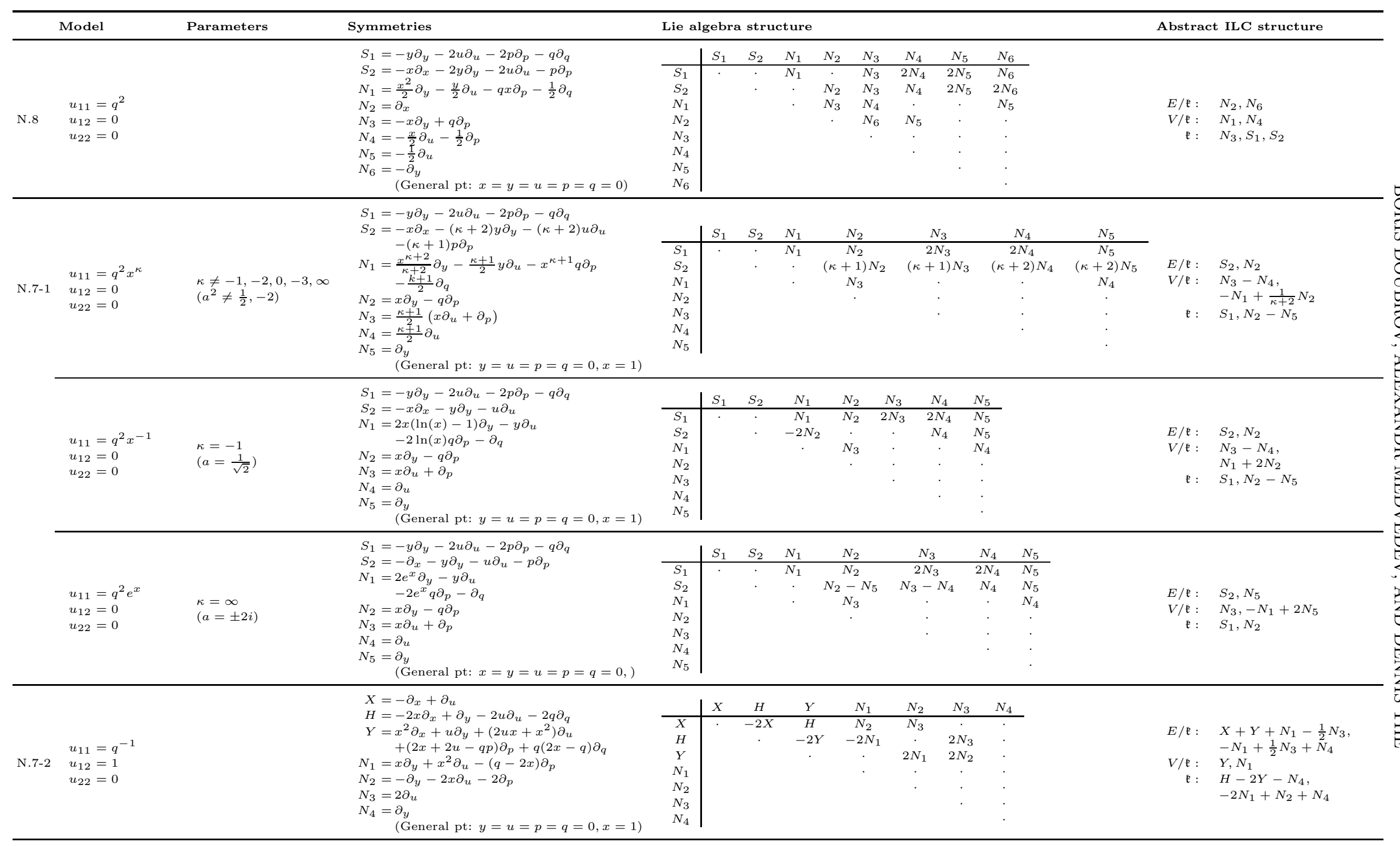


TABle A.1. Classification of type $\mathrm{N}$ cases (continued)

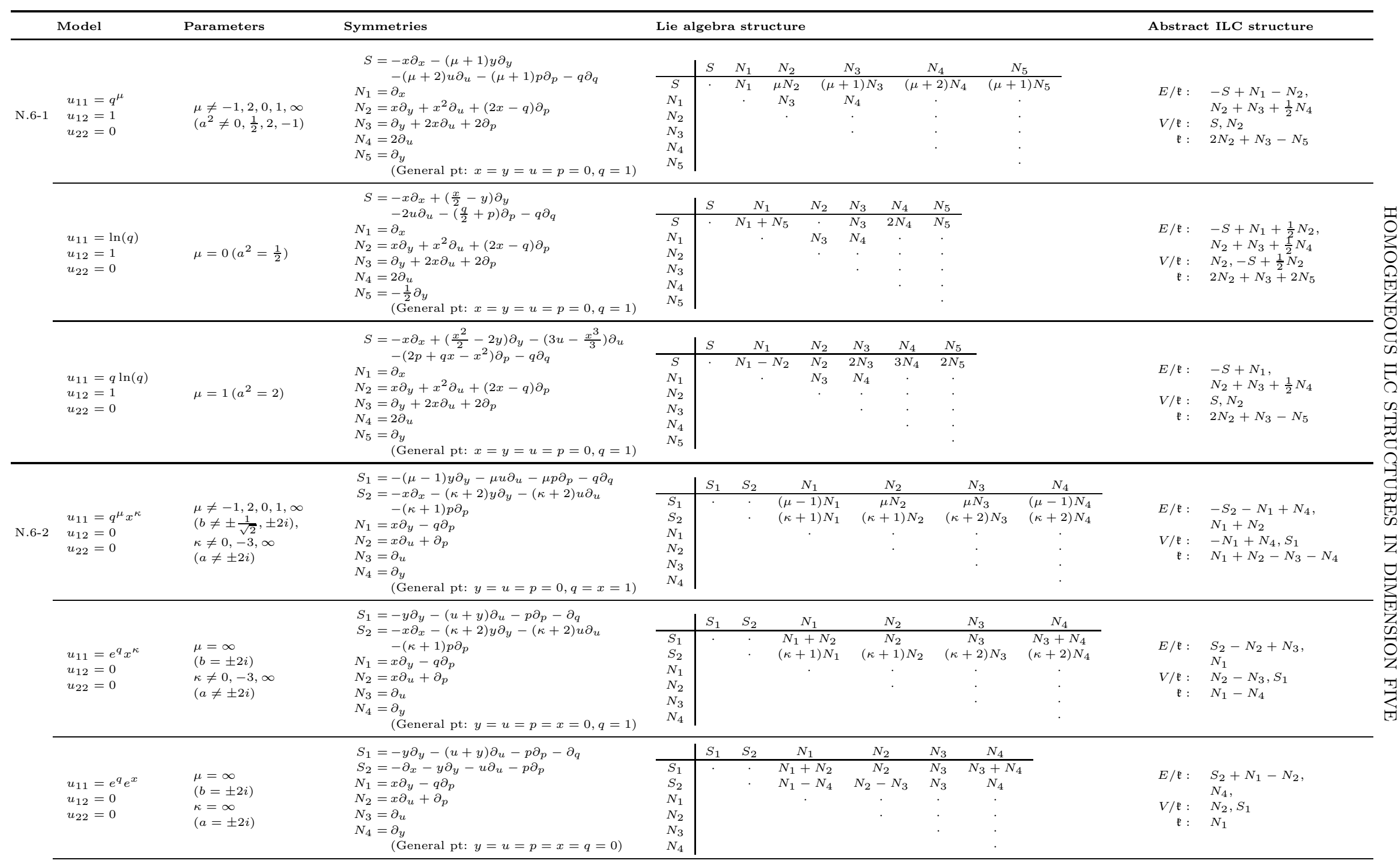


TABle A.1. Classification of type $\mathrm{N}$ cases (continued)

\begin{tabular}{|c|c|c|c|c|c|c|c|c|c|c|c|c|}
\hline Model & Parameters & Symmetries & \multicolumn{8}{|c|}{ Lie algebra structure } & \multicolumn{2}{|c|}{ Abstract ILC structure } \\
\hline $\begin{array}{l}u_{11}=\ln (q) x^{\kappa} \\
u_{12}=0 \\
u_{22}=0\end{array}$ & $\begin{array}{l}\mu=0 \\
\left(b= \pm \frac{1}{\sqrt{2}}\right) \\
\kappa \neq-1,-2,0,-3 \\
\left(a \neq \pm \frac{1}{\sqrt{2}}\right)\end{array}$ & $\begin{aligned} S_{1}= & y \partial_{y}-\frac{x^{\kappa+2}}{(\kappa+1)(\kappa+2)} \partial_{u}-\frac{x^{\kappa+1}}{\kappa+1} \partial_{p}-q \partial_{q} \\
S_{2}= & -x \partial_{x}-(\kappa+2) y \partial_{y}-(\kappa+2) u \partial_{u} \\
& \quad-(\kappa+1) p \partial_{p} \\
N_{1}= & x \partial_{y}-q \partial_{p} \\
N_{2}= & x \partial_{u}+\partial_{p} \\
N_{3}= & \partial_{u} \\
N_{4}= & \partial_{y} \\
& (\text { General pt: } y=u=p=0, q=x=1)\end{aligned}$ & $\begin{array}{l}S_{1} \\
S_{2} \\
N_{1} \\
N_{2} \\
N_{3} \\
N_{4}\end{array}$ & $\mid \begin{array}{l}S_{1} \\
\cdot\end{array}$ & $\begin{array}{c}S_{2} \\
\cdot\end{array}$ & $\begin{array}{r}N_{1} \\
-N_{1} \\
(\kappa+1) \\
\cdot\end{array}$ & & $\frac{N_{2}}{(\kappa+1) N_{2}}$ & $\begin{array}{c}N_{3} \\
\cdot(\kappa+2) N_{3} \\
\vdots\end{array}$ & $\begin{array}{c}N_{4} \\
-N_{4} \\
(\kappa+2) N_{4} \\
\cdot \\
\cdot \\
\cdot\end{array}$ & $\begin{array}{r}E / \mathfrak{k}: \\
V / \mathfrak{k}: \\
\\
\mathfrak{k}:\end{array}$ & $\begin{array}{l}S_{2}, N_{1}+N_{2} \\
-N_{1}+N_{4} \\
S_{1}+\frac{1}{\kappa+1} N_{2}-\frac{1}{\kappa+2} N_{3} \\
N_{1}+N_{2}-N_{3}-N_{4}\end{array}$ \\
\hline $\begin{array}{l}u_{11}=\ln (q) x^{-2} \\
u_{12}=0 \\
u_{22}=0\end{array}$ & $\begin{array}{l}\mu=0 \\
\left(b= \pm \frac{1}{\sqrt{2}}\right) \\
\kappa=-2 \\
\left(a= \pm \frac{1}{\sqrt{2}}\right)\end{array}$ & $\begin{array}{l}S_{1}=y \partial_{y}+\ln (x) \partial_{u}+\frac{1}{x} \partial_{p}-q \partial_{q} \\
S_{2}=-x \partial_{x}+p \partial_{p} \\
N_{1}=x \partial_{y}-q \partial_{p} \\
N_{2}=x \partial_{u}+\partial_{p} \\
N_{3}=\partial_{u} \\
N_{4}=\partial_{y} \\
\quad(\text { General pt: } y=u=p=0, q=x=1)\end{array}$ & $\begin{array}{l}S_{1} \\
S_{2} \\
N_{1} \\
N_{2} \\
N_{3} \\
N_{4}\end{array}$ & $S_{1}$ & $\begin{array}{l}S_{2} \\
N_{3} \\
\cdot\end{array}$ & $\begin{array}{c}N_{1} \\
-N_{1} \\
-N_{1} \\
.\end{array}$ & $\begin{array}{c}N_{2} \\
\cdot N_{2} \\
\cdot\end{array}$ & $\begin{array}{cr}N_{3} & I \\
\cdot & - \\
& \\
\cdot & \\
\cdot & \end{array}$ & $\begin{array}{c}N_{4} \\
-N_{4} \\
\cdot \\
\cdot \\
\cdot \\
\cdot\end{array}$ & & $\begin{array}{r}E / \mathfrak{k}: \\
V / \mathfrak{k}: \\
\mathfrak{k}:\end{array}$ & $\begin{array}{l}S_{2}, N_{1}+N_{2} \\
N_{1}-N_{4} \\
S_{1}-N_{2}+N_{3} \\
N_{1}+N_{2}-N_{3}-N_{4}\end{array}$ \\
\hline
\end{tabular}

TABLE A.2. Classification of type D cases

\begin{tabular}{|c|c|c|c|c|c|c|c|c|c|c|c|c|c|c|c|}
\hline \multirow{3}{*}{ D. 7} & \multirow[b]{2}{*}{$\begin{array}{l}\text { Model } \\
\begin{array}{l} \\
u_{11}=p^{2} \\
u_{12}=0 \\
u_{22}=\lambda q^{2}\end{array}\end{array}$} & \multirow{2}{*}{$\begin{array}{l}\text { Parameters } \\
\\
\\
\begin{array}{l}\lambda \neq 0,-1 \\
(a \neq \pm 3 / 4)\end{array}\end{array}$} & \multirow[b]{2}{*}{$\begin{array}{l}\text { Symmetries } \\
\begin{aligned} X_{1} & =\partial_{x} \\
H_{1} & =-2 x \partial_{x}+\partial_{u}+2 p \partial_{p} \\
Y_{1} & =-x^{2} \partial_{x}+x \partial_{u}+(1+2 x p) \partial_{p} \\
X_{2} & =\partial_{y} \\
H_{2} & =-2 y \partial_{y}+\frac{1}{\lambda} \partial_{u}+2 q \partial_{q} \\
Y_{2}= & -y^{2} \partial_{y}+\frac{1}{\lambda} y \partial_{u}+\left(\frac{1}{\lambda}+2 y q\right) \partial_{q} \\
Z & =\partial_{u} \\
& (\text { General pt: } x=y=u=p=q=0)\end{aligned}\end{array}$} & \multicolumn{9}{|c|}{ Lie algebra structure } & \multicolumn{3}{|c|}{ Abstract ILC structure } \\
\hline & & & & $\begin{array}{c}X_{1} \\
H_{1} \\
Y_{1} \\
X_{2} \\
H_{2} \\
Y_{2} \\
Z\end{array}$ & \begin{tabular}{|c|}
$X_{1}$ \\
$\cdot$
\end{tabular} & $\begin{array}{r}H \\
-22\end{array}$ & & $\begin{array}{c}Y_{1} \\
H_{1} \\
-2 Y_{1}\end{array}$ & $\begin{array}{c}X_{2} \\
\\
\vdots\end{array}$ & $\begin{array}{c}\mathrm{H}_{2} \\
\cdot \\
\cdot \\
-2 \mathrm{X} \\
\cdot\end{array}$ & & $\begin{array}{c}Y_{2} \\
\cdot \\
\cdot \\
H_{2} \\
-2 H_{2} \\
\cdot\end{array}$ & $\begin{array}{l}Z \\
\cdot \\
\cdot \\
\cdot \\
\cdot \\
. \\
.\end{array}$ & $\begin{array}{r}\mathfrak{k}: \\
E / \mathfrak{k}: \\
V / \mathfrak{k}:\end{array}$ & $\begin{array}{l}H_{1}-Z, \lambda H_{2}-Z \\
X_{1}, X_{2} \\
Y_{1}, Y_{2}\end{array}$ \\
\hline & $\begin{array}{l}u_{11}=p^{2} \\
u_{12}=0 \\
u_{22}=0\end{array}$ & $\begin{array}{l}\lambda=0 \\
(a= \pm 3 / 4)\end{array}$ & $\begin{aligned} X_{1} & =\partial_{x} \\
H_{1} & =-2 x \partial_{x}+\partial_{u}+2 p \partial_{p} \\
Y_{1} & =-x^{2} \partial_{x}+x \partial_{u}+(1+2 x p) \partial_{p} \\
S & =-y \partial_{y}+q \partial_{q} \\
X_{2} & =\partial_{y} \\
Y_{2} & =y \partial_{u}+\partial_{q} \\
Z & =\partial_{u} \\
& (\text { General pt: } x=y=u=p=q=0)\end{aligned}$ & $\begin{array}{c}X_{1} \\
H_{1} \\
Y_{1} \\
S \\
X_{2} \\
Y_{2} \\
Z\end{array}$ & \begin{tabular}{|c|}
$X_{1}$ \\
$\cdot$
\end{tabular} & $\frac{H}{-22}$ & & $\begin{array}{c}Y_{1} \\
H_{1} \\
-2 Y_{1}\end{array}$ & $\begin{array}{l}S \\
\\
\dot{5} \\
\cdot\end{array}$ & $\begin{array}{c}X_{2} \\
\cdot \\
\cdot \\
\cdot \\
X_{2} \\
\cdot\end{array}$ & $\begin{array}{c}Y_{2} \\
\cdot \\
\cdot \\
-Y_{2} \\
Z\end{array}$ & $\begin{array}{l}Z \\
\cdot \\
\cdot \\
\cdot \\
\cdot \\
. \\
. \\
.\end{array}$ & & $\begin{array}{r}\mathfrak{k}: \\
E / \mathfrak{k}: \\
V / \mathfrak{k}:\end{array}$ & $\begin{array}{l}H_{1}-Z, S \\
X_{1}, X_{2} \\
Y_{1}, Y_{2}\end{array}$ \\
\hline D. 6-1 & $\begin{array}{l}u_{11}=p^{2}-q^{4} / 4 \\
u_{12}=q\left(p-q^{2} / 2\right) \\
u_{22}=p-q^{2} / 2\end{array}$ & & $\begin{aligned} X_{1}= & \partial_{x} \\
H_{1}= & -2 x \partial_{x}-y \partial_{y}+\partial_{u}+2 p \partial_{p}+q \partial_{q} \\
Y_{1}= & -x^{2} \partial_{x}-x y \partial_{y}+\left(x+y^{2} / 2\right) \partial_{u} \\
& +(1+2 x p+y q) \partial_{p}+(x q+y) \partial_{q} \\
X_{2}= & \partial_{y} \\
Y_{2}= & x \partial_{y}-y \partial_{u}-q \partial_{p}-\partial_{q} \\
Z= & -\partial_{u} \\
& (\text { General pt: } x=y=u=p=q=0)\end{aligned}$ & $\begin{array}{c}X_{1} \\
H_{1} \\
Y_{1} \\
X_{2} \\
Y_{2} \\
Z\end{array}$ & $X_{1}$ & $\begin{array}{r}H \\
-2 . \\
.\end{array}$ & & $\begin{array}{c}Y_{1} \\
H_{1} \\
-2 Y_{1}\end{array}$ & $\begin{array}{l}X_{2} \\
\dot{\cdot} \\
X_{2} \\
Y_{2} \\
\cdot\end{array}$ & $\begin{array}{c}Y_{2} \\
X_{2} \\
-Y_{2} \\
\cdot \\
Z \\
\cdot\end{array}$ & $\begin{array}{l}Z \\
\cdot \\
\cdot \\
\cdot \\
\cdot \\
\cdot\end{array}$ & 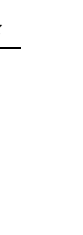 & & $\begin{array}{r}\mathfrak{k}: \\
E / \mathfrak{k}: \\
V / \mathfrak{k}:\end{array}$ & $\begin{array}{l}H_{1}+Z \\
X_{1}, X_{2} \\
Y_{1}, Y_{2}\end{array}$ \\
\hline D.6-2 & $\begin{array}{l}u_{11}=p^{\mu} \\
u_{12}=0 \\
u_{22}=0\end{array}$ & $\begin{array}{l}\mu \neq 0,1,2, \infty \\
(a \neq 1,2 / 3,4 / 3)\end{array}$ & $\begin{aligned} S_{1} & =-y \partial_{y}+q \partial_{q} \\
S_{2} & =-\frac{\mu-1}{\mu-2} x \partial_{x}-y \partial_{y}-u \partial_{u}+\frac{1}{\mu-2} p \partial_{p} \\
X & =\partial_{y} \\
Y & =y \partial_{u}+\partial_{q} \\
Z & =\partial_{u} \\
Z_{1} & =\partial_{x} \\
& (\text { General pt: } x=y=u=q=0, p=1)\end{aligned}$ & \begin{tabular}{c|}
$S_{1}$ \\
$S_{2}$ \\
$X$ \\
$Y$ \\
$Z$ \\
$Z_{1}$
\end{tabular} & $S_{1}$ & $S_{2}$ & $\begin{array}{l}X \\
X \\
X \\
\cdot\end{array}$ & $\begin{array}{c}Y \\
-Y \\
\dot{\cdot} \\
Z\end{array}$ & $\begin{array}{l}Z \\
\cdot \\
z \\
\cdot \\
\cdot\end{array}$ & $\begin{array}{c}Z_{1} \\
\frac{\mu-1}{\mu-2} Z_{1} \\
\cdot \\
\cdot\end{array}$ & & & & $\begin{array}{r}\mathfrak{k}: \\
E / \mathfrak{k}: \\
V / \mathfrak{k}:\end{array}$ & $\begin{array}{l}S_{1} \\
(\mu-2) S_{2}+Z+Z_{1}, X \\
S_{2}, Y\end{array}$ \\
\hline
\end{tabular}


TABle A.2. Classification of type D cases

\begin{tabular}{|c|c|c|c|c|c|c|c|c|c|c|c|c|c|}
\hline & \multirow{2}{*}{$\begin{array}{l}\text { Model } \\
\\
u_{11}=\exp (p) \\
u_{12}=0 \\
u_{22}=0\end{array}$} & \multirow{2}{*}{$\begin{array}{l}\text { Parameters } \\
\\
\\
\mu=\infty \\
(a=4 / 3)\end{array}$} & \multirow{2}{*}{$\begin{array}{l}\text { Symmetries } \\
\begin{aligned} S_{1} & =-y \partial_{y}+q \partial_{q} \\
S_{2} & =-x \partial_{x}-y \partial_{y}-(u-x) \partial_{u}+\partial_{p} \\
X & =\partial_{y} \\
Y & =y \partial_{u}+\partial_{q} \\
Z & =\partial_{u} \\
Z_{1} & =\partial_{x} \\
& (\text { General pt: } x=y=u=p=q=0)\end{aligned}\end{array}$} & \multicolumn{8}{|c|}{ Lie algebra structure } & \multicolumn{2}{|c|}{ Abstract ILC structure } \\
\hline & & & & \begin{tabular}{c|}
$S_{1}$ \\
$S_{2}$ \\
$X$ \\
$Y$ \\
$Z$ \\
$Z_{1}$
\end{tabular} & $S_{1}$ & $\begin{array}{c}S_{2} \\
\cdot\end{array}$ & $\begin{array}{l}X \\
X \\
X\end{array}$ & $\begin{array}{c}Y \\
-Y \\
\dot{Z} \\
\dot{Z}\end{array}$ & $\begin{array}{l}Z \\
\dot{Z} \\
\dot{\cdot} \\
\dot{\cdot}\end{array}$ & $\begin{array}{c}Z_{1} \\
-Z+Z_{1} \\
\dot{\cdot} \\
\dot{\cdot}\end{array}$ & & $\begin{array}{r}\mathfrak{k}: \\
E / \mathfrak{k}: \\
V / \mathfrak{k}:\end{array}$ & $\begin{array}{l}S_{1} \\
S_{2}+Z_{1}, X \\
S_{2}, Y\end{array}$ \\
\hline \multirow[t]{2}{*}{ D.6-3 } & $\begin{array}{l}u_{11}=\lambda p^{2} \frac{\sqrt{u-p q}}{u^{3 / 2}} \\
u_{12}=1+\lambda(p q-2 u) \frac{\sqrt{u-p q}}{u^{3 / 2}} \\
u_{22}=\lambda q^{2} \frac{\sqrt{u-p q}}{u^{3 / 2}}\end{array}$ & $\begin{array}{l}\lambda \neq 0, \pm 1 / 2 \\
(a \neq 0, \pm 3)\end{array}$ & $\begin{aligned} X_{1}= & \partial_{x} \\
H_{1}= & -2 x \partial_{x}-2 u \partial_{u}-2 q \partial_{q} \\
Y_{1}= & -x^{2} \partial_{x}-u \partial_{y}-2 x u \partial_{u}+ \\
& (p q-2 u) \partial_{p}+\left(q^{2}-2 x q\right) \partial_{q} \\
X_{2}= & \partial_{y} \\
H_{2}= & -2 y \partial_{y}-2 u \partial_{u}-2 p \partial_{p} \\
Y_{2}= & -u \partial_{x}-y^{2} \partial_{y}-2 y u \partial_{u}+ \\
& \left(p^{2}-2 p y\right) \partial_{p}+(p q-2 u) \partial_{q} \\
& (\text { General pt: } x=y=p=q=0, u=1)\end{aligned}$ & \begin{tabular}{c|}
$X_{1}$ \\
$H_{1}$ \\
$Y_{1}$ \\
$X_{2}$ \\
$H_{2}$ \\
$Y_{2}$
\end{tabular} & $X_{1}$ & $\begin{array}{r}H_{1} \\
-2 X\end{array}$ & $\begin{array}{ll}1 \\
X_{1}-\end{array}$ & $\begin{array}{c}Y_{1} \\
H_{1} \\
-2 Y_{1}\end{array}$ & $\begin{array}{c}X_{2} \\
\cdot \\
\cdot\end{array}$ & $\begin{array}{c}H_{2} \\
\cdot \\
\cdot \\
-2 X_{2} \\
\cdot\end{array}$ & $\begin{array}{c}Y_{2} \\
\cdot \\
\cdot \\
\cdot \\
H_{2} \\
-2 Y_{2} \\
\cdot\end{array}$ & $\begin{array}{r}\mathfrak{k}: \\
E / \mathfrak{k}: \\
V / \mathfrak{k}:\end{array}$ & $\begin{array}{l}H_{1}-H_{2} \\
X_{1}+\frac{2 \lambda-1}{2 \lambda+1} Y_{2} \\
X_{2}+\frac{2 \lambda-1}{2 \lambda+1} Y_{1} \\
X_{1}+Y_{2}, X_{2}+Y_{1}\end{array}$ \\
\hline & $\begin{array}{l}u_{11}=p^{2} \sqrt{1-2 p q} \\
u_{12}=(p q-1) \sqrt{1-2 p q} \\
u_{22}=q^{2} \sqrt{1-2 p q}\end{array}$ & $(a= \pm 3)$ & $\begin{aligned} X & =-u \partial_{x}-y \partial_{u}+p^{2} \partial_{p}+(p q-1) \partial_{q} \\
H & =-x \partial_{x}+y \partial_{y}+p \partial_{p}-q \partial_{q} \\
Y & =-u \partial_{y}-x \partial_{u}+(p q-1) \partial_{p}+q^{2} \partial_{q} \\
E_{1} & =\partial_{x} \\
E_{2}= & \partial_{u} \\
E_{3}= & \partial_{y} \\
& \quad(\text { General pt: } x=y=u=0, p=q=1)\end{aligned}$ & \begin{tabular}{|c|}
$X$ \\
$H$ \\
$Y$ \\
$E_{1}$ \\
$E_{2}$ \\
$E_{3}$
\end{tabular} & $X$ & $\frac{H}{-X}$ & $\begin{array}{c}Y \\
H \\
-Y \\
\cdot\end{array}$ & $\begin{array}{c}E_{1} \\
\cdot \\
E_{1} \\
E_{2}\end{array}$ & $\begin{array}{l}E_{2} \\
E_{1} \\
\dot{\cdot} \\
E_{3}\end{array}$ & $\begin{array}{c}E_{3} \\
E_{2} \\
-E_{3} \\
\cdot \\
\cdot \\
\cdot\end{array}$ & & $\begin{array}{r}\mathfrak{k}: \\
E / \mathfrak{k}: \\
V / \mathfrak{k}:\end{array}$ & $\begin{array}{l}H \\
X+E_{1}, Y+E_{2} \\
X, Y\end{array}$ \\
\hline D.6-4 & $\begin{array}{l}u_{11}=0 \\
u_{12}=\frac{1+p q}{u} \\
u_{22}=0\end{array}$ & & $\begin{aligned} X_{1}= & \partial_{x} \\
H_{1}= & -2 x \partial_{x}-u \partial_{u}+p \partial_{p}-q \partial_{q} \\
Y_{1}= & -x^{2} \partial_{x}-x u \partial_{u}+(x p-u) \partial_{p}-x q \partial_{q} \\
X_{2}= & \partial_{y} \\
H_{2}= & -2 y \partial_{y}-u \partial_{u}-p \partial_{p}+q \partial_{q} \\
Y_{2}= & -y^{2} \partial_{y}-y u \partial_{u}-y p \partial_{p}+(y q-u) \partial_{q} \\
& (\text { General pt: } x=y=p=q=0, u=1)\end{aligned}$ & $\begin{array}{c} \\
X_{1} \\
H_{1} \\
Y_{1} \\
X_{2} \\
H_{2} \\
Y_{2}\end{array}$ & $X_{1}$ & $\begin{array}{r}H_{1} \\
-2 X \\
\end{array}$ & X & $\begin{array}{c}Y_{1} \\
H_{1} \\
-2 Y_{1}\end{array}$ & $X_{2}$ & $\begin{array}{c}\mathrm{H}_{2} \\
\\
\cdot \\
-2 X_{2}\end{array}$ & $\begin{array}{c}Y_{2} \\
\cdot \\
\cdot \\
\cdot \\
H_{2} \\
-2 Y_{2}\end{array}$ & $\begin{array}{r}\mathfrak{k}: \\
E / \mathfrak{k}: \\
V / \mathfrak{k}:\end{array}$ & $\begin{array}{l}H_{1}-H_{2} \\
X_{1}-Y_{2}, X_{2}-Y_{1} \\
Y_{1}, Y_{2}\end{array}$ \\
\hline
\end{tabular}

TABle A.3. Classification of type III cases

\begin{tabular}{|c|c|c|c|c|c|c|c|c|c|c|c|}
\hline \multirow[b]{2}{*}{ III.6-1 } & \multirow{2}{*}{$\begin{array}{l}\text { Model } \\
\\
\\
u_{11}=p /(x-q) \\
u_{12}=0 \\
u_{22}=0\end{array}$} & \multirow[b]{2}{*}{ 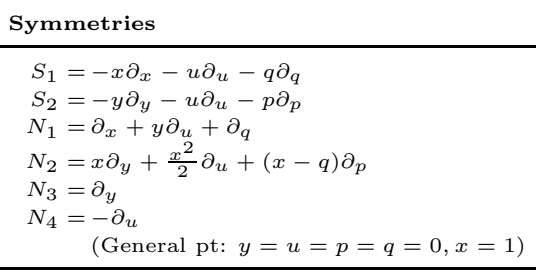 } & \multicolumn{7}{|c|}{ Lie algebra structure } & \multicolumn{2}{|c|}{ Abstract ILC structure } \\
\hline & & & $\begin{array}{l} \\
S_{1} \\
S_{2} \\
N_{1} \\
N_{2} \\
N_{3} \\
N_{4}\end{array}$ & $S_{1}$ & $\begin{array}{c}S_{2} \\
\\
\cdot\end{array}$ & $\begin{array}{c}N_{1} \\
N_{1} \\
\cdot \\
\cdot\end{array}$ & $\begin{array}{c}N_{2} \\
-N_{2} \\
N_{2} \\
N_{3} \\
\cdot\end{array}$ & $\begin{array}{c}N_{3} \\
\cdot \\
N_{3} \\
N_{4}\end{array}$ & $\begin{array}{c}N_{4} \\
N_{4} \\
N_{4} \\
\cdot \\
\cdot \\
\cdot \\
\cdot\end{array}$ & $\begin{array}{r}\mathfrak{k}: \\
E / \mathfrak{k}: \\
V / \mathfrak{k}:\end{array}$ & $\begin{array}{l}S_{2} \\
S_{1}, N_{3} \\
S_{1}+N_{1}, N_{2}-N_{3}+\frac{1}{2} N_{4}\end{array}$ \\
\hline III.6-2 & $\begin{array}{l}u_{11}=2 q(2 p-q u) \\
u_{12}=q^{2} \\
u_{22}=0\end{array}$ & $\begin{aligned} X= & \partial_{x} \\
H= & -2 x \partial_{x}-y \partial_{y}+u \partial_{u}+3 p \partial_{p}+2 q \partial_{q} \\
Y= & -x^{2} \partial_{x}-x y \partial_{y}+(u x+y) \partial_{u} \\
& +(3 p x+u+q y) \partial_{p}+(2 q x+1) \partial_{q} \\
S= & -y \partial_{y}-u \partial_{u}-p \partial_{p} \\
N_{1}= & \partial_{y} \\
N_{2}= & x \partial_{y}-\partial_{u}-q \partial_{p} \\
& (\text { General pt: } x=y=u=q=0, p=1)\end{aligned}$ & $\begin{array}{c}X \\
H \\
Y \\
S \\
N_{1} \\
N_{2}\end{array}$ & $\mid x$ & $\begin{array}{c}H \\
-2 X\end{array}$ & $\begin{array}{c}Y \\
H \\
-2 Y \\
\cdot\end{array}$ & $\begin{array}{l}S \\
\\
\cdot \\
\cdot\end{array}$ & $\begin{array}{c}N_{1} \\
\cdot \\
N_{1} \\
N_{2} \\
N_{1} \\
\cdot\end{array}$ & $\begin{array}{c}N_{2} \\
N_{1} \\
-N_{2} \\
\cdot \cdot \\
N_{2} \\
\cdot \\
\cdot\end{array}$ & $\begin{array}{r}\mathfrak{k}: \\
E / \mathfrak{k}: \\
V / \mathfrak{k}:\end{array}$ & $\begin{array}{l}H+3 S \\
X-N_{2}, N_{1} \\
S, Y\end{array}$ \\
\hline
\end{tabular}




\begin{tabular}{|c|c|c|}
\hline & Parameters change & Basis change \\
\hline N.8 & & $S_{1}=-\frac{1}{2} e_{7}-\frac{3}{2} e_{8}, S_{1}=-\frac{1}{2} e_{8}-\frac{3}{2} e_{7}, N_{1}=\frac{1}{2} e_{4}, N_{2}=-2 e_{1}, N_{3}=-e_{6}, N_{4}=-\frac{1}{2} e_{3}, N_{5}=-e_{5}, N_{6}=-2 e_{2}$ \\
\hline \multirow[t]{3}{*}{ N.7-1 } & $\begin{array}{l}\kappa=-\frac{3}{2}+\frac{3 a}{2 \sqrt{a^{2}+4}}, \kappa \neq-1,-2, \infty \\
a=\frac{2 \kappa+3}{\sqrt{-\kappa^{2}-3 \kappa}}, a \neq \pm \frac{1}{\sqrt{2}}, \pm 2 i\end{array}$ & $\begin{array}{l}S_{1}=-\frac{1}{2} e_{6}, S_{2}=\frac{1}{\sqrt{a^{2}+4}} e_{1}-\frac{3 a}{2 \sqrt{a^{2}+4}} e_{6}, N_{1}=-\frac{1}{\sqrt{a^{2}+4}\left(3 a+\sqrt{a^{2}+4}\right)}\left(e_{2}+\left(2 a^{2}-1\right) e_{4}+2 a e_{7}\right), N_{2}=-\frac{1}{2\left(2 a^{2}-1\right)}\left(e_{2}+\frac{a+\sqrt{a^{2}+4}}{2} e_{7}\right) \\
N_{3}=\frac{3 a-\sqrt{a^{2}+4}}{8\left(a^{2}+4\right)^{\frac{3}{2}}}\left(\frac{a+\sqrt{a^{2}+4}}{2} e_{3}+e_{5}\right), N_{4}=\frac{3 a-\sqrt{a^{2}+4}}{8\left(a^{2}+4\right)^{\frac{3}{2}}}\left(\frac{a-\sqrt{a^{2}+4}}{2} e_{3}+e_{5}\right), N_{5}=-\frac{1}{2\left(2 a^{2}-1\right)}\left(e_{2}+\frac{a-\sqrt{a^{2}+4}}{2} e_{7}\right)\end{array}$ \\
\hline & $\begin{array}{l}\kappa=-1 \\
a=\frac{1}{\sqrt{2}}\end{array}$ & $\begin{array}{l}S_{1}=-\frac{1}{2} e_{6}, S_{2}=\frac{\sqrt{2}}{3} e_{1}-\frac{1}{2} e_{6}, N_{1}=\frac{2}{9} e_{2}-e_{4}-\frac{\sqrt{2}}{9} e_{7}, N_{2}=-\frac{1}{9} e_{2}-\frac{\sqrt{2}}{9} e_{7} \\
N_{3}=\frac{\sqrt{2}}{9} e_{3}+\frac{1}{9} e_{5}, N_{4}=-\frac{1}{9 \sqrt{2}} e_{3}+\frac{1}{9} e_{5}, N_{5}=-\frac{1}{9} e_{2}+\frac{1}{9 \sqrt{2}} e_{7}\end{array}$ \\
\hline & $\begin{array}{l}\kappa=\infty \\
a=2 i\end{array}$ & $\begin{array}{l}S_{1}=-\frac{1}{2} e_{6}, S_{2}=\frac{i}{3} e_{1}-e_{6}, N_{1}=-\frac{i}{3} e_{2}+3 i e_{4}-\frac{4}{3} e_{7}, N_{2}=-\frac{1}{2} e_{7} \\
N_{3}=-\frac{3 i}{2} e_{3}, N_{4}=-\frac{i}{2} e_{3}+\frac{1}{2} e_{5}, N_{5}=-\frac{i}{6} e_{2}-\frac{1}{6} e_{7}\end{array}$ \\
\hline N.7-2 & & $\begin{array}{l}X=-e_{1}-\frac{1}{2} e_{2}+e_{4}+\frac{1}{2} e_{5}+2 e_{6}+\frac{1}{2} e_{7}, H=-2 e_{4}-\frac{1}{2} e_{5}-2 e_{6}-\frac{1}{2} e_{7}, Y=-e_{4}, \\
N_{1}=\frac{1}{2} e_{3}, N_{2}=e_{3}+\frac{1}{2} e_{5}-\frac{1}{2} e_{7}, N_{3}=-e_{2}+e_{3}+e_{5}-e_{7}, N_{4}=-\frac{1}{2} e_{5}-\frac{1}{2} e_{7}\end{array}$ \\
\hline \multirow[t]{3}{*}{ N.6-1 } & $\begin{array}{l}\mu=\frac{2 a^{2}-1}{a^{2}+1} \neq 0,1 \\
a^{2}=\frac{1+\mu}{2-\mu} \neq \frac{1}{2}, 2\end{array}$ & $\begin{array}{l}S=\frac{1}{a} e_{4}, N_{1}=-\frac{a}{a^{2}+1} e_{1}-\frac{a^{2}+1}{a\left(2 a^{2}-1\right)\left(a^{2}-2\right)} e_{3}+\frac{1}{a} e_{4}+\frac{2\left(a^{2}-1\right)}{\left(2 a^{2}-1\right)\left(a^{2}-2\right)} e_{6}, N_{2}=-\frac{1}{\left(2 a^{2}-1\right)\left(a^{2}-2\right)}\left(\frac{a^{2}+1}{a} e_{3}+a^{2} e_{6}\right), \\
N_{3}=\frac{1}{\left(2 a^{2}-1\right)\left(a^{2}-2\right)}\left(\frac{a^{3}}{a^{2}+1} e_{2}+\frac{a^{2}+2}{a} e_{3}-e_{5}+\frac{1}{a^{2}+1} e_{6}\right), N_{4}=\frac{1}{\left(2 a^{2}-1\right)\left(a^{2}-2\right)}\left(\frac{2 a}{a^{2}+1} e_{2}-\frac{2}{a} e_{3}+2 e_{5}-\frac{2}{a^{2}+1} e_{6}\right), \\
N_{5}=\frac{1}{\left(2 a^{2}-1\right)\left(a^{2}-2\right)}\left(\frac{a^{3}}{a^{2}+1} e_{2}-a e_{3}-e_{5}-\frac{2 a^{2}+1}{a^{2}+1} e_{6}\right)\end{array}$ \\
\hline & $\mu=0, a^{2}=\frac{1}{2}$ & $\begin{array}{l}S=\frac{\sqrt{2}}{3} e_{3}+\sqrt{2} e_{4}-\frac{1}{9} e_{6}, N_{1}=-\frac{\sqrt{2}}{3} e_{1}+\sqrt{2} e_{4}-\frac{2}{3} e_{6}, N_{2}=\frac{2 \sqrt{2}}{3} e_{3}-\frac{2}{9} e_{6}, N_{3}=-\frac{2 \sqrt{2}}{27} e_{2}-\frac{10 \sqrt{2}}{9} e_{3}+\frac{4}{9} e_{5}-\frac{8}{27} e_{6}, \\
N_{4}=-\frac{8 \sqrt{2}}{27} e_{2}+\frac{8 \sqrt{2}}{9} e_{3}-\frac{8}{9} e_{5}+\frac{16}{27} e_{6}, N_{5}=\frac{\sqrt{2}}{27} e_{2}-\frac{\sqrt{2}}{9} e_{3}-\frac{2}{9} e_{5}-\frac{8}{27} e_{6}\end{array}$ \\
\hline & $\mu=1, a^{2}=2$ & $\begin{array}{l}S=\frac{1}{\sqrt{2}} e_{4}, N_{1}=-\frac{\sqrt{2}}{3} e_{1}+\frac{1}{\sqrt{2}} e_{4}+\frac{1}{3} e_{6}, N_{2}=-\frac{1}{3 \sqrt{2}} e_{3}+\frac{2}{9} e_{6}, N_{3}=\frac{2 \sqrt{2}}{27} e_{2}+\frac{2 \sqrt{2}}{9} e_{3}-\frac{1}{9} e_{5}+\frac{1}{27} e_{6} \\
N_{4}=\frac{2 \sqrt{2}}{27} e_{2}-\frac{\sqrt{2}}{9} e_{2}+\frac{2}{9} e_{5}-\frac{2}{27} e_{6}, N_{5}=\frac{2 \sqrt{2}}{27} e_{2}-\frac{\sqrt{2}}{9} e_{3}-\frac{1}{9} e_{5}-\frac{5}{27} e_{6}\end{array}$ \\
\hline \multirow[t]{5}{*}{ N.6-2 } & $\begin{array}{l}\mu=\frac{1}{2}+\frac{3 b}{2 \sqrt{b^{2}+4}}, \quad b=\frac{2 \mu-1}{\sqrt{-\mu^{2}+\mu+2}}, \\
\kappa=-\frac{3}{2}+\frac{3 a}{2 \sqrt{a^{2}+4}}, a=\frac{2 \kappa+3}{\sqrt{-\kappa^{2}-3 \kappa}} \\
b \neq \pm \frac{1}{\sqrt{2}}, \pm 2 i, \mu \neq 0,1, \infty \\
a \neq \pm 2 i, \kappa \neq \infty\end{array}$ & $\begin{array}{l}S_{1}=\frac{-1}{\sqrt{b^{2}+4}} e_{4}, S_{2}=\frac{-1}{\sqrt{a^{2}+4}} e_{1}+\frac{1}{\left(1-2 b^{2}\right) \sqrt{a^{2}+4}} e_{3}+\frac{2 b}{\left(1-2 b^{2}\right) \sqrt{a^{2}+4}} e_{6}, \\
N_{1}=\frac{1}{\left(2 b^{2}-1\right)\left(a^{2}+4\right)}\left(\frac{-b-\sqrt{b^{2}+4}}{2} e_{2}+\frac{a+\sqrt{a^{2}+4}}{2} e_{3}-e_{5}+\frac{\left(a+\sqrt{a^{2}+4}\right)\left(\sqrt{b^{2}+4}+b\right)}{4} e_{6}\right), \\
N_{2}=\frac{1}{\left(2 b^{2}-1\right)\left(a^{2}+4\right)}\left(\frac{b-\sqrt{b^{2}+4}}{2} e_{2}-\frac{a+\sqrt{a^{2}+4}}{2} e_{3}+e_{5}+\frac{\left(a+\sqrt{a^{2}+4}\right)\left(\sqrt{b^{2}+4}-b\right)}{4} e_{6}\right), \\
N_{3}=\frac{1}{\left(2 b^{2}-1\right)\left(a^{2}+4\right)}\left(\frac{b-\sqrt{b^{2}+4}}{2} e_{2}-\frac{a-\sqrt{a^{2}+4}}{2} e_{3}+e_{5}+\frac{\left(a-\sqrt{a^{2}+4}\right)\left(\sqrt{b^{2}+4}-b\right)}{4} e_{6}\right), \\
N_{4}=\frac{1}{\left(2 b^{2}-1\right)\left(a^{2}+4\right)}\left(\frac{-b-\sqrt{b^{2}+4}}{2} e_{2}+\frac{a-\sqrt{a^{2}+4}}{2} e_{3}-e_{5}+\frac{\left(a-\sqrt{a^{2}+4}\right)\left(\sqrt{b^{2}+4}+b\right)}{4} e_{6}\right),\end{array}$ \\
\hline & $\begin{array}{l}\mu=\infty, b=2 i, \kappa=-\frac{3}{2}+\frac{3 a}{2 \sqrt{a^{2}+4}} \\
a=\frac{2 \kappa+3}{\sqrt{-\kappa^{2}-3 \kappa}}, a \neq \pm 2 i, \kappa \neq \infty\end{array}$ & $\begin{array}{l}S_{1}=\frac{i}{3} e_{4}, S_{2}=\frac{-1}{\sqrt{a^{2}+4}}\left(e_{1}-\frac{1}{9} e_{3}-\frac{4 i}{9} e_{6}\right), N_{1}=-\frac{i}{3\left(a^{2}+4\right)}\left(e_{2}-\frac{a+\sqrt{a^{2}+4}}{2} e_{6}\right), N_{2}=\frac{1}{9\left(a^{2}+4\right)}\left(i e_{2}-\frac{a+\sqrt{a^{2}+4}}{2} e_{3}+e_{5}-\frac{i\left(a+\sqrt{a^{2}+4}\right)}{2} e_{6}\right) \\
N_{3}=\frac{1}{9\left(a^{2}+4\right)}\left(i e_{2}+\frac{\sqrt{a^{2}+4}-a}{2} e_{3}+e_{5}+\frac{i\left(\sqrt{a^{2}+4}-a\right)}{2} e_{6}\right), N_{4}=-\frac{i}{3\left(a^{2}+4\right)}\left(e_{2}+\frac{\sqrt{a^{2}+4}-a}{2} e_{6}\right)\end{array}$ \\
\hline & $\begin{array}{l}\mu=\infty, \quad b= \pm 2 i \\
\kappa=\infty, \quad a= \pm 2 i\end{array}$ & $S_{1}=-\frac{i}{3} e_{4}, S_{2}=-\frac{i}{3} e_{1}+\frac{i}{27} e_{3}+\frac{4}{27} e_{6}, N_{1}=-\frac{1}{9} e_{6}, N_{2}=\frac{i}{27} e_{3}+\frac{1}{27} e_{6}, N_{3}=-\frac{i}{81} e_{2}+\frac{i}{81} e_{3}+\frac{1}{81} e_{5}+\frac{1}{81} e_{6}, N_{4}=\frac{i}{27} e_{2}-\frac{1}{27} e_{6}$ \\
\hline & $\begin{array}{l}\mu=0, \quad b=\frac{1}{\sqrt{2}}, \kappa=-\frac{3}{2}+\frac{3 a}{2 \sqrt{a^{2}+4}} \\
a=\frac{2 \kappa+3}{\sqrt{-\kappa^{2}-3 \kappa}}, a \neq \pm 2 i, \kappa \neq \infty\end{array}$ & $\begin{array}{l}S_{1}=\frac{\sqrt{2}}{3} e_{4}+\frac{2}{9\left(2 a^{2}-1\right)}\left(\sqrt{2} e_{2}-2 a e_{3}+e_{5}-2 \sqrt{2} a e_{6}\right), S_{2}=-\frac{1}{\sqrt{a^{2}+4}}\left(e_{1}+\frac{\sqrt{2}}{3} e_{6}\right), \\
N_{1}=-\frac{1}{9\left(a^{2}+4\right)}\left(\sqrt{2} e_{2}-2 e_{5}+\left(a+\sqrt{a^{2}+4}\right)\left(e_{3}-\frac{1}{\sqrt{2}} e_{6}\right)\right), N_{2}=-\frac{1}{9\left(a^{2}+4\right)}\left(2 \sqrt{2} e_{2}+2 e_{5}+\left(\sqrt{a^{2}+4}+a\right)\left(e_{3}+\sqrt{2} e_{6}\right)\right), \\
N_{3}=-\frac{1}{9\left(a^{2}+4\right)}\left(2 \sqrt{2} e_{2}+2 e_{5}+\left(\sqrt{a^{2}+4}-a\right)\left(e_{3}+\sqrt{2} e_{6}\right)\right), N_{4}=-\frac{1}{9\left(a^{2}+4\right)}\left(\sqrt{2} e_{2}-2 e_{5}+\left(a-\sqrt{a^{2}+4}\right)\left(e_{3}-\frac{1}{\sqrt{2}} e_{6}\right)\right)\end{array}$ \\
\hline & $\begin{array}{l}\mu=0, b=\frac{1}{\sqrt{2}} \\
\kappa=-2, a=-\frac{1}{\sqrt{2}}\end{array}$ & $\begin{array}{l}S_{1}=\frac{2 \sqrt{2}}{27} e_{3}+\frac{\sqrt{2}}{3} e_{4}+\frac{4}{27} e_{6}, S_{2}=-\frac{\sqrt{2}}{3} e_{1}-\frac{2}{9} e_{6}, N_{1}=-\frac{2 \sqrt{2}}{81} e_{2}-\frac{2 \sqrt{2}}{81} e_{3}+\frac{4}{81} e_{5}+\frac{2}{81} e_{6}, N_{2}=-\frac{4 \sqrt{2}}{81} e_{2}+\frac{2 \sqrt{2}}{81} e_{3}-\frac{4}{81} e_{5}+\frac{4}{81} e_{6} \\
N_{3}=-\frac{4}{81}\left(\sqrt{2} e_{2}+\sqrt{2} e_{3}+e_{5}+2 e_{6}\right), N_{4}=-\frac{2 \sqrt{2}}{81}\left(e_{2}-2 e_{3}-\sqrt{2} e_{5}+\sqrt{2} e_{6}\right)\end{array}$ \\
\hline
\end{tabular}


TABLE A.4. Basis change from Cartan reduced basis to adapted Lie algebra basis (continued)

\begin{tabular}{|c|c|c|}
\hline & Parameters change & Basis change \\
\hline \multirow[t]{2}{*}{ D.7 } & $\begin{array}{l}\lambda=\frac{3+4 a}{3-4 a}, a \neq \pm \frac{3}{4} \\
a=\frac{3}{4} \frac{\lambda-1}{\lambda+1}, \lambda \neq 0,-1\end{array}$ & $\begin{array}{l}X_{1}=\frac{2}{4 a-3} e_{1}, H_{1}=-\frac{1}{4 a-3}\left(2 e_{5}+2(2 a-1) e_{6}-(2 a-3) e_{7}\right), Y_{1}=e_{3}, \quad X_{2}=-e_{2}, H_{2}=\frac{1}{4 a+3}\left(2 e_{5}-2(2 a+1) e_{6}-(2 a+3) e_{7}\right), Y_{2}=\frac{2}{4 a+3} e_{4} \\
Z=-\frac{1}{4 a-3}\left(2 e_{5}+e_{6}+2 a e_{7}\right)\end{array}$ \\
\hline & $a= \pm \frac{3}{4}, \lambda=0$ & $X_{1}=-\frac{1}{3} e_{4}, H_{1}=-\frac{1}{3} e_{5}+\frac{5}{6} e_{6}+\frac{3}{4} e_{7}, Y_{1}=e_{2}, \quad S=\frac{1}{2}\left(e_{6}-e_{7}\right), X_{2}=-\frac{1}{3} e_{3}, Y_{2}=e_{1}, \quad Z=-\frac{1}{3} e_{5}-\frac{1}{6} e_{6}-\frac{1}{4} e_{7}$ \\
\hline D.6-1 & & $X_{1}=\frac{1}{\sqrt{2}} e_{1}, H_{1}=-\frac{1}{2} e_{5}+\frac{1}{8} e_{6}, Y_{1}=\frac{1}{\sqrt{2}} e_{3}, \quad X_{2}=\sqrt{2} e_{2}, Y_{2}=-\sqrt{2} e_{4}, Z=\frac{1}{2} e_{5}+\frac{3}{8} e_{6}$ \\
\hline \multirow[t]{2}{*}{ D.6-2 } & $\begin{array}{l}\mu=\frac{6(a-1)}{3 a-4}, a \neq 1,2 / 3,4 / 3 \\
a=\frac{2}{3} \frac{2 \mu-3}{\mu-2}, \mu \neq 0,1,2, \infty\end{array}$ & $\begin{array}{l}S_{1}=\frac{1}{2} e_{6}, S_{2}=-\frac{3 a-2}{12}\left(2(a-1) e_{4}+3 e_{6}\right), X=e_{1}, Y=-\frac{(a-1)(3 a-2)}{(3 a-4)^{2}} e_{3}, Z=-\frac{1}{(3 a-4)^{2}}\left(6 e_{2}+\frac{2(a-1)(3 a-2)}{3} e_{4}-(a-1)(3 a-2) e_{5}-\frac{9 a^{2}-15 a-2}{4} e_{6}\right), \\
Z_{1}=\frac{1}{(3 a-4)^{2}}\left(3(3 a-2) e_{2}+\frac{(a-1)(3 a-2)^{2}}{3} e_{4}-(a-1)(3 a-2) e_{5}+\frac{9(a-1)(3 a-2)}{4} e_{6}\right)\end{array}$ \\
\hline & $a=4 / 3, \mu=\infty$ & $S_{1}=-\frac{1}{2} e_{6}, S_{2}=-\frac{1}{9} e_{4}-\frac{1}{2} e_{6}, X=-\frac{1}{6} e_{3}, Y=e_{1}, Z=\frac{3}{2} e_{2}+\frac{1}{9} e_{4}-\frac{1}{6} e_{5}+\frac{3}{8} e_{6}, Z_{1}=\frac{3}{2} e_{2}+\frac{1}{9} e_{4}+\frac{3}{2} e_{6}$ \\
\hline \multirow[t]{2}{*}{ D.6-3 } & $\begin{array}{l}\lambda=\frac{3}{2 \sqrt{9-a^{2}}}, a \neq 0, \pm 3 \\
a=\frac{3 \sqrt{4 \lambda^{2}-1}}{2 \lambda}, \lambda \neq 0, \pm 1 / 2\end{array}$ & $\begin{array}{l}X_{1}=\lambda e_{1}+\frac{\lambda(2 \lambda-1)}{\sqrt{4 \lambda^{2}-1}} e_{4}, H_{1}=\frac{4 \lambda}{3} e_{5}+e_{6}, Y_{1}=-\frac{2}{3} \sqrt{4 \lambda^{2}-1} e_{2}-\frac{2}{3}(2 \lambda+1) e_{3} \\
X_{2}=\frac{2}{3} \sqrt{4 \lambda^{2}-1} e_{2}+\frac{2}{3}(2 \lambda+1) e_{3}, H_{2}=\frac{4 \lambda}{3} e_{5}-e_{6}, Y_{2}=-\lambda e_{1}-\frac{\lambda(2 \lambda+1)}{\sqrt{4 \lambda^{2}-1}} e_{4}\end{array}$ \\
\hline & $a= \pm 3$ & $X=-e_{4}, H=e_{6}, Y=-\frac{2}{3} e_{3}, \quad E_{1}=e_{1}+e_{4}, E_{2}=-\frac{2}{3} e_{5}, E_{3}=\frac{2}{3}\left(e_{2}+e_{3}\right)$ \\
\hline D.6-4 & & $X_{1}=-2 e_{1}+\frac{1}{3} e_{4}, H_{1}=\frac{2}{3} e_{5}+e_{6}, Y_{1}=\frac{1}{3} e_{3}, \quad X_{2}=-2 e_{2}+\frac{1}{3} e_{3}, H_{2}=\frac{2}{3} e_{5}-e_{6}, Y_{2}=\frac{1}{3} e_{4}$ \\
\hline III.6-1 & & $S_{1}=2 e_{1}+\frac{5}{4} e_{6}, S_{2}=-\frac{1}{2} e_{6}, N_{1}=-2 e_{1}+e_{4}-\frac{9}{4} e_{6}, N_{2}=-\frac{1}{2} e_{2}+\frac{1}{8} e_{3}+\frac{1}{2} e_{5}, N_{3}=-e_{2}, N_{4}=-e_{2}+\frac{3}{4} e_{3}-e_{5}$ \\
\hline III.6-2 & & $X=2 e_{1}+e_{5}, H=\frac{3}{2} e_{3}+\frac{5}{2} e_{6}, Y=-2 e_{4}, S=-\frac{1}{2}\left(e_{3}+e_{6}\right), N_{1}=-\frac{1}{2} e_{2}, N_{2}=e_{5}$ \\
\hline
\end{tabular}

TABLE A.5. Basis change which reflects redundancy in parameters

\begin{tabular}{lll}
\hline \multicolumn{1}{c}{ Parameters change } & Basis change \\
\hline N.7-1 & $a \rightarrow-a,(\kappa \rightarrow-\kappa-3)$, & $\left(e_{1}, e_{2}, e_{3}, e_{4}, e_{5}, e_{6}\right) \rightarrow\left(-e_{1}, e_{2},-e_{3}, e_{4}, e_{5},-e_{6}\right)$ \\
\hline N.6-1 & $a \rightarrow-a$ & $\left(e_{1}, e_{2}, e_{3}, e_{4}, e_{5}, e_{6}\right) \rightarrow\left(-e_{1},-e_{2},-e_{3},-e_{4}, e_{5}, e_{6}\right)$ \\
\hline N.6-2 & $a \rightarrow-a,(\kappa \rightarrow-\kappa-3)$, & $\left(e_{1}, e_{2}, e_{3}, e_{4}, e_{5}, e_{6}\right) \rightarrow\left(-e_{1}, e_{2},-e_{3}, e_{4}, e_{5},-e_{6}\right)$ \\
& $b \rightarrow-b,(\mu \rightarrow-\mu+1)$ & $\left(e_{1}, e_{2}, e_{3}, e_{4}, e_{5}, e_{6}\right) \rightarrow\left(e_{1},-e_{2}, e_{3},-e_{4}, e_{5},-e_{6}\right)$ \\
\hline D.7 & $a \rightarrow-a,(\lambda \rightarrow 1 / \lambda)$ & $\left(e_{1}, e_{2}, e_{3}, e_{4}, e_{5}, e_{6}, e_{7}\right) \rightarrow\left(e_{2}, e_{1}, e_{4}, e_{3}, e_{5}, e_{6},-e_{7}\right)$ \\
\hline D.6-3 & $a \rightarrow-a,(\lambda \rightarrow-\lambda)$ & $\left(e_{1}, e_{2}, e_{3}, e_{4}, e_{5}, e_{6}\right) \rightarrow\left(e_{2},-e_{1}, e_{4},-e_{3}, e_{5},-e_{6}\right)$ \\
\hline
\end{tabular}


Table A.6. Duality

\begin{tabular}{|c|c|c|}
\hline & Parameters change & Basis change \\
\hline N.8 & (self-dual) & $\left(e_{1}, e_{2}, e_{3}, e_{4}, e_{5}, e_{6}, e_{7}, e_{8}\right) \rightarrow\left(e_{4}, e_{3}, e_{2}, e_{1},-e_{5},-e_{6}, e_{8}, e_{7}\right)$ \\
\hline N.7-2 & (self-dual) & $\left(e_{1}, e_{2}, e_{3}, e_{4}, e_{5}, e_{6}, e_{7}\right) \rightarrow\left(e_{4}, e_{3}, e_{2}, e_{1},-e_{5},-e_{6},-e_{7}\right)$ \\
\hline N.6-1 & (self-dual) & $\left(e_{1}, e_{2}, e_{3}, e_{4}, e_{5}, e_{6}\right) \rightarrow\left(\frac{a^{2}+1}{a^{2}} e_{4}, \frac{a^{2}+1}{a^{2}} e_{3}, \frac{a^{2}}{a^{2}+1} e_{2}, \frac{a^{2}}{a^{2}+1} e_{1},-e_{5},-e_{6}\right)$ \\
\hline N.6-2 & $(a, b) \rightarrow(b, a),(\mu, \kappa) \rightarrow(\kappa+2, \mu-2)$ & $\left(e_{1}, e_{2}, e_{3}, e_{4}, e_{5}, e_{6}\right) \rightarrow\left(e_{4}, e_{3}, e_{2}, e_{1},-e_{5},-e_{6}\right)$ \\
\hline D.7 & (self-dual) & $\left(e_{1}, e_{2}, e_{3}, e_{4}, e_{5}, e_{6}, e_{7}\right) \rightarrow\left(e_{3}, e_{4}, e_{1}, e_{2},-e_{5},-e_{6},-e_{7}\right)$ \\
\hline D.6-1 & (self-dual) & $\left(e_{1}, e_{2}, e_{3}, e_{4}, e_{5}, e_{6}\right) \rightarrow\left(e_{3}, e_{4}, e_{1}, e_{2},-e_{5},-e_{6}\right)$ \\
\hline D.6-2 & (self-dual) & $\left(e_{1}, e_{2}, e_{3}, e_{4}, e_{5}, e_{6}\right) \rightarrow\left(e_{3},-\frac{(a-1)(3 a-2)}{9} e_{4}, e_{1},-\frac{9}{(a-1)(3 a-2)} e_{2},-e_{5},-e_{6}\right)$ \\
\hline D.6-3 & (self-dual) & $\left(e_{1}, e_{2}, e_{3}, e_{4}, e_{5}, e_{6}\right) \rightarrow\left(e_{3}, e_{4}, e_{1}, e_{2},-e_{5},-e_{6}\right)$ \\
\hline
\end{tabular}


Faculty of Mathematics and Mechanics, Belarusian State University, Nezavisimosti ave. 4, 220050, Minsk, Belarus

E-mail address: doubrov@bsu.by

School of Science and Technology, University of New England, Armidale NSW 2351, Australia Current address: International School for Advanced Studies, via Bonomea 265, Trieste 34136, Italy

E-mail address: amedvedev@sissa.it

Mathematical Sciences Institute, Australian National University, ACT 0200, Australia Current address: Fakultät für Mathematik, Universität Wien, Oskar-Morgenstern Platz 1, 1090 Wien, Austria E-mail address: dennis.the@univie.ac.at 